\title{
The ability of intermediate-band Strömgren photometry to correctly identify dwarf, subgiant, and giant stars and provide stellar metallicities and surface gravities ${ }^{\star}$
}

\author{
A. S. Árnadóttir, S. Feltzing, and I. Lundström
}

\begin{abstract}
Lund Observatory, Department of Astronomy and Theoretical Physics, Lund University, Box 43, 22100 Lund, Sweden e-mail: [anna, sofia,ingemar] @astro.lu.se
\end{abstract}

Received 25 October 2009 / Accepted 10 February 2010

\section{ABSTRACT}

\begin{abstract}
Context. Several large scale photometric and spectroscopic surveys are being undertaken to provide a more detailed picture of the Milky Way. Given the necessity of generalisation in the determination of, e.g., stellar parameters when tens and hundred of thousands of stars are considered it remains important to provide independent, detailed studies to verify the methods used in the surveys. Aims. Our first aim is to critically evaluate available calibrations for deriving $[\mathrm{M} / \mathrm{H}]$ from Strömgren photometry. Secondly, we develop the standard sequences for dwarf stars to reflect their inherent metallicity dependence. Finally, we test how well metallicities derived from ugriz photometry reproduce metallicities derived from the well-tested system of Strömgren photometry.

Methods. We evaluate available metallicity calibrations based on Strömgren $u v b y$ photometry for dwarf stars using a catalogue of stars with both uvby photometry and spectroscopically determined iron abundances $([\mathrm{Fe} / \mathrm{H}])$. The catalogue was created for this project. Using this catalogue, we also evaluate available calibrations that determine $\log g$. A larger catalogue, in which metallicity is determined directly from uvby photometry, is used to trace metallicity-dependent standard sequences for dwarf stars. We also perform comparisons, for both dwarf and giant stars, of metallicities derived from ugriz photometry with metallicities derived from Strömgren photometry.

Results. We provide a homogenised catalogue of 451 dwarf stars with $0.3<(b-y)_{0}<1.0$. All stars in the catalogue have uvby photometry and $[\mathrm{Fe} / \mathrm{H}]$ determined from spectra with high resolution and high signal-to-noise ratios $(\mathrm{S} / \mathrm{N})$. Using this catalogue, we test how well various photometric metallicity calibrations reproduce the spectroscopically determined $[\mathrm{Fe} / \mathrm{H}]$. Using the preferred metallicity calibration for dwarf stars, we derive new standard sequences in the $c_{1,0}$ versus $(b-y)_{0}$ plane and in the $c_{1,0}$ versus $(v-y)_{0}$ plane for dwarf stars with $0.40<(b-y)_{0}<0.95$ and $1.10<(v-y)_{0}<2.38$.

Conclusions. We recommend the calibrations of Ramírez \& Meléndez (2005) in deriving metallicities from Strömgren photometry and find that intermediate band photometry, such as Strömgren photometry, more accurately than broad band photometry reproduces spectroscopically determined $[\mathrm{Fe} / \mathrm{H}]$. Strömgren photometry is also better at differentiating between dwarf and giant stars. We conclude that additional investigations of the differences between metallicities derived from ugriz photometry and intermediate-band photometry, such as Strömgren photometry, are required.
\end{abstract}

Key words. stars: abundances - stars: fundamental parameters - solar neighborhood - Galaxy: stellar content

\section{Introduction}

The photometric system introduced by Bengt Strömgren (Strömgren 1963, 1964) provides a means of reliably estimating stellar parameters for stars with a wide range of spectral classes. For instance, metallicities can be determined for many types of stars. In particular, the system can accurately identify stars at different evolutionary stages (see discussion in, e.g., Strömgren 1963). This makes it possible to determine the distances of stars with no parallax measurements. If reddening is not known, the system must, however, be complemented with $\mathrm{H} \beta$ photometry.

The advent of CCD photometry has meant that larger and deeper areas of sky can be scanned to determine the properties of stars in the field and from them infer the properties of the stellar populations in the Milky Way. For broad-band photometry, this approach has been very successful, e.g., Gilmore \& Reid (1983), who inferred the existence of the thick disk and,

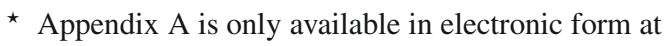
http://www . aanda.org, Appendix B is available in electronic form at http://www.aanda.org and at the CDS via anonymous ftp to cdsarc.u-strasbg.fr (130.79.128.5) or via

http://cdsarc.u-strasbg.fr/viz-bin/qcat?J/A+A/521/A40 e.g., Ibata et al. (2001) and Ferguson et al. (2005), who studied the stellar structures in the Andromeda galaxy. Arguably the most important large study of this kind is the Sloan Digital Sky Survey (SDSS) (York et al. 2000), which provides deep photometry of stars for roughly half the sky.

In contrast, the usage of narrow and medium band photometry for Galactic studies was for a much longer time severely hampered by the relative inefficiency of the CCDs, which required too long exposure times to make these techniques competitive. This combined with relatively small fields of view (mainly due to small filters on the cameras equipped with suitable filters) meant that only very small portions of the sky could be usefully studied. Additionally, the size of telescopes that have cameras with Strömgren filters and relatively low efficiency in the blue also hampered observations in the $u$ filter (e.g., von Hippel 1992). All of this meant that systems, such as that designed by Strömgren, were mainly applied to the study of globular and open clusters (two fairly recent examples are provided by Grundahl et al. 2002; Twarog et al. 2003) or to individual stars (e.g., Olsen 1994b, 1995; Schuster \& Nissen 1989a; Schuster et al. 2004, 2006). Recent attempts to use Strömgren photometry to study the properties of the Milky Way stellar disks away from the solar 
neighbourhood are few. Interesting examples being von Hippel \& Bothun (1993) and Jønch-Sørensen (1995).

Advancements in technology have meant that we now also have access to larger $\mathrm{CCD}$ areas on telescopes equipped with large $u v b y$-filters, enabling an efficient study of stellar properties across larger areas of the sky.

We have published two studies based on Strömgren photometry of the red giant branches of dwarf spheroidal galaxies in the Local Group using the Wide Field Camera (WFC) on the Isaac Newton Telescope on La Palma (Faria et al. 2007; Adén et al. 2009a). This camera is equipped with large filters that allow an, almost, unvignetted field of view of half by half a degree. However, far more can be achieved with this dataset. It provides the largest database of Strömgren photometry for Milky Way disk stars without any kinematic or colour biases. The stars are situated at distances between 0.5 and $4 \mathrm{kpc}$ away from the Sun and in the directions of the four dwarf spheroidal galaxies Draco, Sextans, Hercules, and Ursa Major II. We intend to apply this unique dataset to explore the properties of the Milky Way disk(s) in some detail.

As part of a series of papers on the properties of the Milky Way disks using Strömgren photometry, we have undertaken a critical evaluation of the available calibrations for metallicity and $\log g$ determinations for dwarf and sub-giant stars. We have also determined new standard sequences (compare, e.g., Olsen 1984) to improve the identification of dwarf and giant stars in the distant disk and halo. We also provide a basic comparison of metallicities derived using Strömgren photometry and metallicities derived for dwarf and giant stars from SDSS ugriz photometry using the calibration in Ivezić et al. (2008).

The paper is organised as follows. Section 2 provides a short introduction to the Strömgren photometric system and background to the work presented here, Sect. 3 details the catalogues we compile to test the metallicity calibrations available for dwarf stars, which is described in Sect. 4 where we also compare the Strömgren metallicities with those derived by the SDSS project (DR7 Abazajian et al. 2009). Section 5 considers the system's ability to distinguish between giant and dwarf stars of similar colour. We also provide new, metallicity-dependent stellar sequences for dwarf stars in this section. These new sequences are compared to model predictions (e.g., isochrones) in Sect. 6. Section 7 summarises our findings and provides a few suggestions for future work.

\section{A short introduction to the Strömgren photometric system}

The Strömgren system consists of the four medium-width filters $u, v, b$, and $y$ (hereafter collectively denoted as $u v b y$ ), where the $y$ magnitude is calibrated to be the same as the $V$ magnitude in the $U B V$ system (e.g., Johnson \& Morgan 1953; see also Olsen 1984 and Fig. 1). The filters are centred on 350, 410, 470, and $550 \mathrm{~nm}$ and their half-widths are 38, 20, 10, and $20 \mathrm{~nm}$, respectively (e.g., Golay 1974, page 180). In addition, the system relies on the three colour indices (differences) that are constructed in the following way (compare, e.g., Strömgren 1963)

$$
\begin{aligned}
& (b-y) \\
& m_{1} \equiv(v-b)-(b-y) \\
& c_{1} \equiv(u-v)-(v-b) .
\end{aligned}
$$

These indices are designed to measure important properties of the stars and were first introduced by Bengt Strömgren in a series of papers, including Strömgren (1963) and Strömgren (1964).

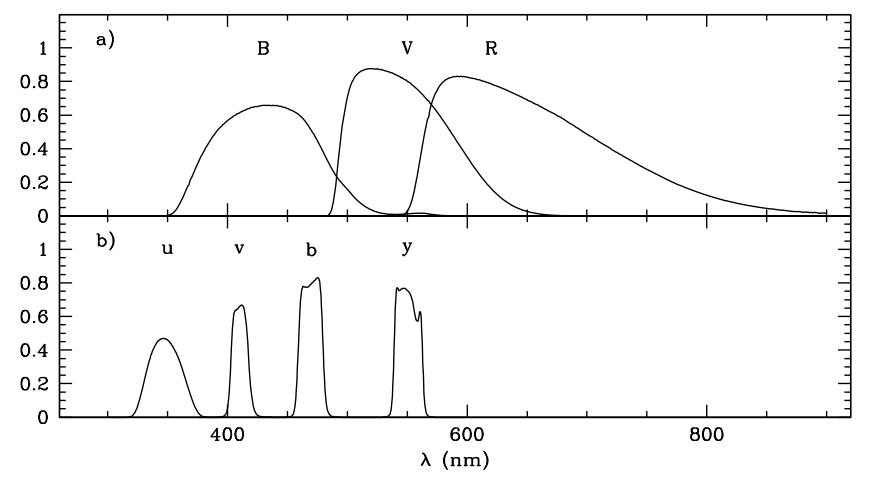

Fig. 1. Filter throughput curves for broad-band and Strömgren filters. Filter curves are from the database of filters used with the wide-field camera on the Isaac Newton Telescope. The database is available at http: //catserver.ing.iac.es/filter/. a) Harris $B, V$, and $R$ filters, and $\mathbf{b})$ Strömgren $u, v, b$, and $y$ filters.

Work on the system continued by establishing standard stars (e.g., Crawford \& Barnes 1970; Grønbech et al. 1976; Olsen 1983; Perry et al. 1987; Olsen 1993). However, as discussed in Clausen et al. (1997), the establishment of standard fields akin to those available for UVBRI photometry (Landolt 1992) have only very recently been attempted. An additional problem is that the primary standards are too bright for most available combinations of cameras with uvby filters and telescopes. Although Clausen et al. (1997), Cousins (1987), and Schuster \& Nissen (1988) provide secondary fainter standards, the situation for both standard fields and secondary standards that can be used with large telescopes remains unsatisfactory.

There are two main sets of established standard stars for the uvby system, those of Bond (1980) and Olsen (1993). There are some non-negligible differences between the two sets and Olsen (1995) provides a detailed discussion of this subject. He concludes that the main difference concerns the $c_{1}$ index and is caused mainly by the $u$-filter. Hence, if we wish to compare results based on the two sets of standards we need to apply corrections (compare, e.g., Fig. 15 in Faria et al. 2007). We adopt observations calibrated to the system established by Olsen (1993).

The system was originally designed to study earlier types of stars (A2 to G2, Strömgren 1963). Later work has, however, shown that the system and its properties can be extended to later types of stars. Particularly important extensions of the application of the system have been presented by Bond (1970) (for metal-poor giants), Gustafsson \& Ardeberg (1978) (for red horizontal branch stars), Olsen (1984) (for G and K dwarf stars), Schuster \& Nissen (1989b) (for metalpoor stars), Anthony-Twarog \& Twarog (1994) (for giants), and Twarog et al. (2007) (for G and K dwarf stars). The theoretical foundations of these extension can be found in, e.g., Bell \& Gustafsson (1978) and Gustafsson \& Bell (1979), and more recently Önehag et al. (2009). Applications to yellow supergiants have also been successful (see, e.g., Arellano Ferro \& Mendoza V. 1993).

The colour-index $(b-y)$ is relatively unaffected by blanketing effects and can thus be used to measure the stellar temperature (if the reddening is known). Recent examples of colourtemperature calibrations are given for dwarf stars by Alonso et al. (1996), and for giant stars by Alonso et al. (1999). Ramírez \& Meléndez (2005b) provide calibrations for both giant and dwarf stars.

In contrast, the $m_{1}$ index is designed specifically to measure the amount of blanketing in a region around $410 \mathrm{~nm}$ (e.g., 
Crawford 1975) or as originally stated by Strömgren (1963) is "a colour difference that is a measure of the total intensity of the metal lines in the $v$-band". It is thus sensitive to the total amount of metals present in the stellar atmosphere. However, it was soon recognised that these metallicity lines in population I stars are strong enough to depend mainly on microturbulence $\left(\xi_{t}\right)$ and less on metallicity. It was later shown that $\xi_{t}$ is not a free parameter and hence the dependence still prevails (see, e.g., discussion in Gustafsson \& Nissen 1972). Because of the properties of the $m_{1}$ index it can be used to derive metallicities for a variety of late-type stars (e.g., F to $\mathrm{K}$ and $\mathrm{V}$ to III). Recent examples of metallicity calibrations include for giants Hilker (2000) and Calamida et al. (2007), and for dwarf stars Olsen (1984), Schuster \& Nissen (1989b), and Holmberg et al. (2007) (see Sect. 4 for a more complete list). The calibrations for giant stars include only linear terms in the different indices and none include $c_{1}$. For dwarf stars, the relations are more complex and less straightforward, including dependencies also, e.g., on the $c_{1}$ index and quadratic terms. The reliability of the metallicity calibrations for dwarf stars is one of the main topics of this paper.

Finally, the $c_{1}$ index is designed to measure the Balmer discontinuity (Strömgren 1963). For early-type stars, B and A, the $c_{1}$ index is a measure of the temperature but for later type stars ( $F$ and $G$ stars) it provides a measure of the surface gravity. Hence, for stars with spectral class later than roughly A, already by design this system is able to identify different types of stars in a reliable way. This was, in fact, the main advantage of the system as it was used in early applications. Note that the identification works equally well if the reddening is known or if all stars can be assumed to suffer from the same amount of reddening. For stars with spectral type later than A, it was possible, by measuring $(b-y)$ and $c_{1}$ and comparing to standard sequences, to determine an absolute magnitude for the star once it had been classified (e.g., Strömgren 1963). It thus became important to develop standard sequences in the $c_{1}$ vs. $(b-y)$ diagram so that stars could be reliably classified according to spectral class and evolutionary stage. We return to the issue of standard sequences for late-type dwarf stars later in this paper.

The ability to classify stars at different evolutionary stages using the uvby system has been elaborated upon. For metal-poor stars, Schuster et al. (2004) developed a finely tuned classification scheme to identify main sequence, turn-off, blue stragglers, red giant, horizontal branch and asymptotic branch stars (see Fig. 2). Adén et al. (2009a) used this classification scheme to successfully trace the faint $(V \sim 21.1)$ horizontal branch of the Hercules dwarf spheroidal galaxy.

The scheme developed in Schuster et al. (2004) extends only to about $(b-y)_{0}{ }^{1}$ of 0.4 for dwarf stars and about 0.6 for giants. However, the ability of the $u v b y$ system to distinguish different evolutionary stages (for all metallicities) improves as we move to redder colours. A simple illustration of this is given in Fig. 2. In this figure, we reproduce the classification scheme of Schuster et al. (2004) and overlay two sets of isochrones by VandenBerg et al. (2006), which use the temperature-colour transformation

\footnotetext{
1 The subscript 0 indicates that the photometry has been dereddened. In the following, we explicitly indicate which photometry has been dereddened and which has not. All metallicity and other calibrations are based on the star's "true" colours, i.e., the dereddened photometry. However, the separation of dwarf and giant stars with the help of the $c_{1}$ index (see, e.g., Fig. 2) is effective using photometry that has not been dereddened as long as both types of stars are represented and all stars are affected by the same amount of reddening. This is, for example, the case for the dwarf spheroidal galaxies.
}
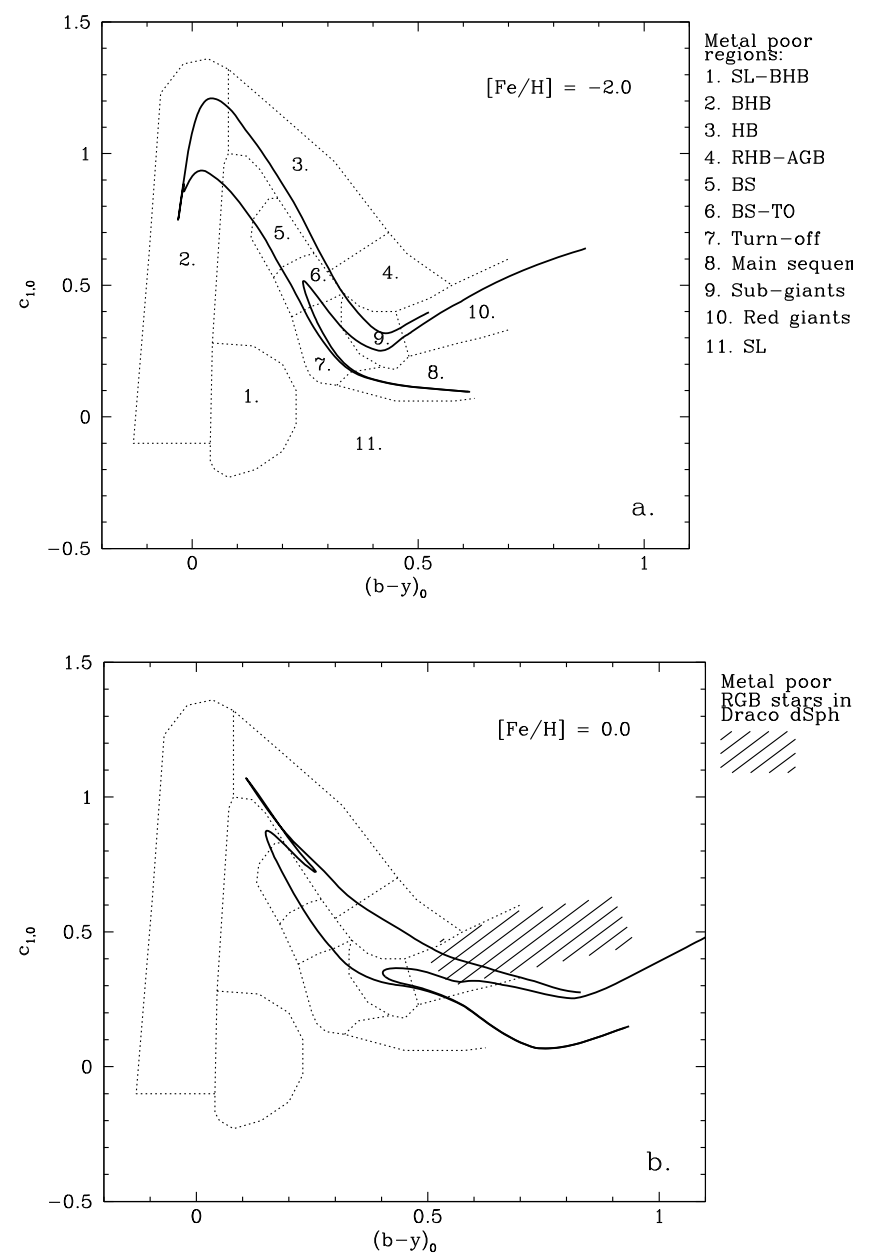

Fig. 2. Illustration of the $u v b y$ system's ability to identify stars at different evolutionary stages. The classification scheme by Schuster et al. (2004) is indicated by dotted lines. Evolutionary stages are identified in panel a) as: 1. SL-BHB: sub-luminous - blue horizontal branch transition; 2. BHB: blue horizontal branch; 3. HB: horizontal branch; 4. RHB-AGB: red horizontal branch - asymptotic giant branch transition; 5. BS: blue-straggler stars; 6. BS-TO: blue-straggler - turn-off transition; 7. Turn-off: turn-off stars; 8. main sequence; 9. sub-giants; 10. red giants; and 11. SL: sub-luminous stars. Two isochrones by VandenBerg et al. (2006) using the temperature-colour transformation by Clem et al. (2004) are shown as full lines (age $=1 \mathrm{Gyr}$ and $10 \mathrm{Gyr}$ ). The metallicities of the isochrones are indicated in the panels. The region occupied by metal-poor red giants in the Draco dwarf spheroidal galaxy (Faria et al. 2007) is indicated by a hashed area in panel b).

by Clem et al. (2004) (but see Faria et al. 2007, for a critical discussion of the reliability of the intermediate metallicity isochrones based on this temperature-colour transformation).

Finally, uvby photometry is often complemented with observations in additional filters. In particular, many studies have been performed using the $\beta$ index (e.g., Schuster et al. 1996). For latetype stars, this index provides a temperature estimate that is essentially independent of reddening. However, the two filters included in this index are both narrow or very narrow, hence for large-scale studies of fainter stars observing times become prohibitively long. Here we are therefore not concerned with the $\beta$ index.

Other studies have also developed systems that use additionally information, e.g., Ca II H and K photometry (see, e.g., Anthony-Twarog \& Twarog 1998). For the same reasons given 
for the $\beta$ index, we do not address these systems but rather consider only uvby, where, in terms of observing time, $u$ is by far the most expensive filter.

\section{Two catalogues}

Before testing available metallicity and $\log g$ calibrations and deriving new standard relations we will first detail how we selected the stars used to perform these tasks. Below we describe the construction of two catalogues for dwarf stars, one with uvby photometry only and one with both uvby photometry and iron abundances determined from high-resolution spectroscopy.

\subsection{Reddening}

For both catalogues, we need to decide whether the photometry for the stars should be dereddened or not and which reddening map to use. We only consider stars that have parallaxes in the Hipparcos catalogue (Perryman et al. 1997; van Leeuwen 2007) and use the same method to deredden the photometry in the two catalogues. In brief, we assume that the dust in the Galactic disk can be modelled as a thin exponential disk with a scale-height of 125 pc (following, e.g., Bonifacio et al. 2000; Beers et al. 2002). Since most of the stars are nearby, they are inside this dust disk. We reduce the extinction accordingly using

$E(B-V)_{\mathrm{star}}=[1-\exp (-|d \sin b| / h)] \cdot E(B-V)_{\mathrm{LOS}}$,

where $E(B-V)_{\text {LOS }}$ is the full colour-excess along the line of sight (LOS) taken from the dust maps by Schlegel et al. (1998), $d$ is the distance (here we use the parallaxes from the new reduction of the Hipparcos catalogue of van Leeuwen 2007), $b$ is the galactic latitude, and $h$ is the scale-height of the thin dust disk (taken to be $125 \mathrm{pc}$, see above).

Following, for instance, Nordström et al. (2004) we assume that stars with $E(B-V)$ below 0.02 are un-reddened and do not apply any dereddening to the photometry for these stars. We discuss the implications of this in Sect. 4.1.

Several studies have noted that the dust maps of Schlegel et al. (1998) overpredict $E(B-V)$ when $E(B-V)>0.15$ (see, e.g., Arce \& Goodman 1999; Beers et al. 2002; Yasuda et al. 2007). Our catalogues are dominated by nearby stars with low $E(B-V)$. For the spectroscopic catalogue discussed in Sect. 3.3 and used to test the metallicity calibration in Sect. 4.1, only two stars have $E(B-V)>0.15$. In the photometric catalogue used to trace dwarf-star sequences in Sect. 5, there are 38 of 3645 stars that have $E(B-V)>0.15$. Since so few stars are affected by a possible overprediction of the reddening we chose not to apply any corrections to the reddening values found from the map by Schlegel et al. (1998).

To deredden the uvby photometry we use the relation for $A_{\lambda} / E(B-V)$ from Table 5 (Col. 8) in Appendix B of Schlegel et al. (1998). For individual magnitudes, this amount to $x_{0}=$ $x-E(B-V) \cdot k_{x}$, where $x$ is any of $u v b y$ and $k_{x}=5.231,4.552$, 4.049 , and 3.277 for $u v b y$, respectively, and the subscript 0 corresponds to the dereddened photometry.

\subsection{The photometric catalogue}

The three studies by Olsen (1993), Olsen (1994a), and Olsen (1994b) represent one of the largest homogeneous catalogues of high quality uvby photometry for nearby dwarf stars that also includes spectral classification of the stars. The stars were classified into three main groups: sub-giant stars (or the BAF

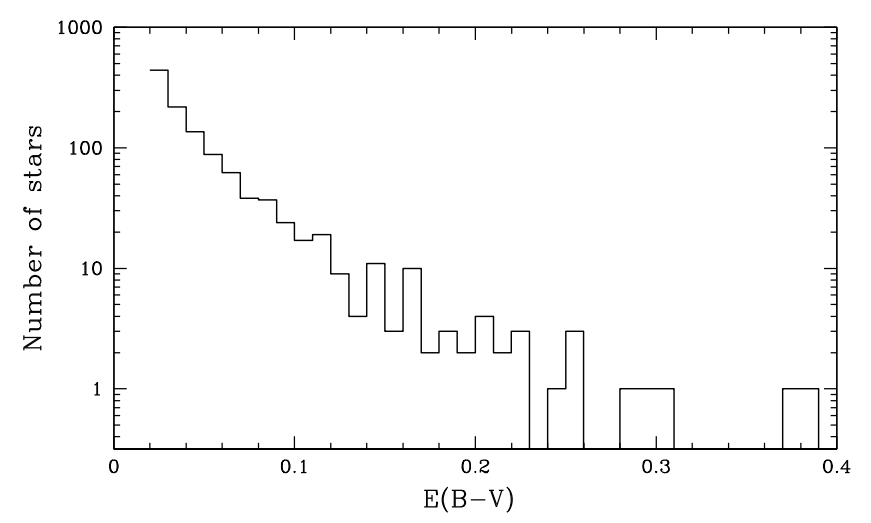

Fig. 3. Distribution of $E(B-V)$ for our photometric catalogue (see Sect. 3.2). There are 2502 stars with $E(B-V)<0.02$, which are not shown.

group), giant stars (or the GKIII group), and dwarf stars (or the GKV group). For our final catalogue, we only include stars classified as dwarf stars by Olsen (the GKV group). Whenever a star has an entry in more than one of the three studies we adopt the most recent set of measurements.

Dereddening was performed as described in Sect. 3.1. The majority of the stars in Olsen (1993), Olsen (1994a), and Olsen (1994b) have parallaxes from Hipparcos (ESA 1997; Perryman et al. 1997; van Leeuwen 2007). Stars that have no parallax from Hipparcos were simply discarded from the photometric catalogue. Known binary stars were excluded using the SIMBAD database. The resulting catalogue consists of 3645 dwarf stars. Figure 4 a shows the distribution of the stars in the HR-diagram.

\subsection{The spectroscopic catalogue}

To test the available metallicity and $\log g$ calibrations for dwarf stars, we need a homogeneous catalogue of stars, which have both $u v b y$ photometry and spectroscopically determined $[\mathrm{Fe} / \mathrm{H}]^{2}$ and $\log g$. The $[\mathrm{Fe} / \mathrm{H}]$ should preferably have been derived using parallaxes, but ionisation equilibrium might also be acceptable (compare discussion in Bensby et al. 2005).

Because we place special emphasis on the redder dwarf stars, we started our search by looking in the General Catalogue of Photometric Data (Mermilliod et al. 1997) for stars with $(b-y)>$ 0.6 , and found such stars in four studies: Olsen (1984), Schuster \& Nissen (1988), Olsen (1993), and Olsen (1994a).

As discussed above, in both Olsen (1993) and Olsen (1994a) the stars were classified according to their evolutionary stages. In these two papers, we found 97 and 29 dwarfs stars, respectively, that are redder than $(b-y)=0.6$. Olsen (1984) and Schuster \& Nissen (1988) do not provide stellar classifications, we therefore used the $c_{1}$ vs. $(b-y)$ diagram, compare Fig. 2, to exclude any obvious giant or early type stars. We found 37 and 27 stars, respectively, in these two papers which are likely dwarf stars with $(b-y)>0.6$.

In total, we found 190 probable dwarf stars with $(b-y)>$ 0.6. Upon further inspection, it was found that 44 entries in this list were duplications. We decided to keep the most recent

\footnotetext{
2 We adopt the usual notation where $[\mathrm{Fe} / \mathrm{H}] \equiv \log \left(N_{\mathrm{Fe}} / N_{\mathrm{H}}\right)_{\star}-$ $\log \left(N_{\mathrm{Fe}} / N_{\mathrm{H}}\right)_{\odot}$ and use $[\mathrm{Fe} / \mathrm{H}]$ exclusively for iron abundances determined from high-resolution spectroscopy. Metallicities determined from photometric calibrations will be either called just that or denoted $[\mathrm{M} / \mathrm{H}]$.
} 
A. S. Árnadóttir et al.: Metallicities and stellar classification from Strömgren photometry
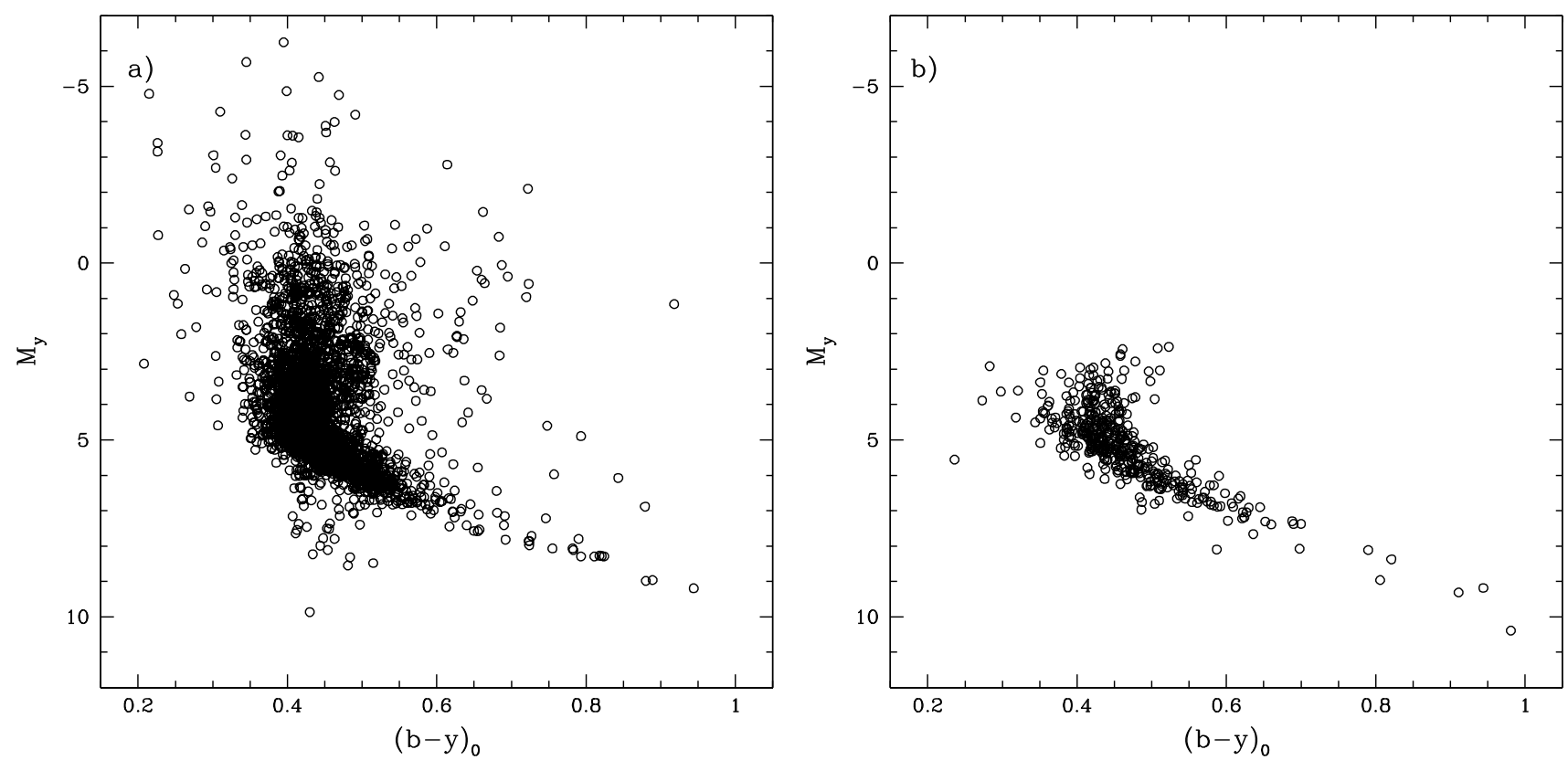

Fig. 4. a) HR diagram for the photometric catalogue of dwarf stars (see Sect. 3.2). b) HR-diagram for the dwarf stars in the spectroscopic catalogue (see Sect. 3.3).

photometric measurements when more than one set of measurements were available for a given star.

Eleven additional stars were excluded ( 5 stars were marked as binaries in one of the four papers and 6 stars had been observed to be variables during those observing campaigns). Finally, we used the SIMBAD database to identify any additional binaries, variables, or unclassified stars. In total, 37 additional stars were excluded by this check: 5 because they had no identification at all in SIMBAD, being possible miss-identifications, 28 stars because they were identified as variable, spectroscopic binaries, carbon stars, T Tauri stars or peculiar; and 4 stars were giants.

For the remaining 98 dwarf stars with $(b-y)>0.6$, we searched the literature for metallicity determinations using the SIMBAD and VizeR databases (Ochsenbein et al. 2000). Fiftyseven of the stars had no previous metallicity determinations at all. Thirteen stars had only metallicities derived from photometry. We were thus left with 28 stars with $(b-y)>0.6$ and $[\mathrm{Fe} / \mathrm{H}]$ derived from high-resolution spectroscopy.

The 28 red dwarf stars were found in 15 studies using highresolution spectroscopy to determine $[\mathrm{Fe} / \mathrm{H}]$ : Valenti \& Fischer (2005), Favata et al. (1997), Feltzing \& Gustafsson (1998), Chen et al. (2000), Thorén \& Feltzing (2000), Santos et al. (2001), Heiter \& Luck (2003), Yong \& Lambert (2003), Santos et al. (2004), Mishenina et al. (2004), Woolf \& Wallerstein (2005), Santos et al. (2005), Luck \& Heiter (2005), Bonfils et al. (2005), and Sousa et al. (2006).

Several of these 15 studies also include large numbers of dwarf stars bluer than $(b-y)=0.6$. This is especially true for Valenti \& Fischer (2005), which includes [Fe/H] for 1040 stars. Our aim is to use our compilation to the test available calibrations for, mainly, F- and G-type dwarf stars. We therefore decided that Valenti \& Fischer (2005) should be the baseline for our compilation.

Following Twarog et al. (2007), the $[\mathrm{Fe} / \mathrm{H}]$ determined in the 15 spectroscopic studies (referred to as the "original studies" below) were moved onto the system of Valenti \& Fischer (2005) in the following way. For each study, we took all stars (i.e., including stars with $(b-y)<0.6)$ in common between the study and Valenti \& Fischer (2005) and performed a least squares fit to determine the coefficients of the equation that transforms $[\mathrm{Fe} / \mathrm{H}]$ onto the metallicity-scale by Valenti \& Fischer (2005) given by

$[\mathrm{Fe} / \mathrm{H}]_{\mathrm{VF} 05}=a[\mathrm{Fe} / \mathrm{H}]+b \log T_{\mathrm{eff}}+c \log g+d$,

where $[\mathrm{Fe} / \mathrm{H}]$ is the iron abundance, $T_{\text {eff }}$ is the effective temperature, and $\log g$ is the surface gravity derived in the original study, that is being moved onto the metallicity-scale by Valenti \& Fischer (2005). $[\mathrm{Fe} / \mathrm{H}]_{\mathrm{VF} 05}$ is the $[\mathrm{Fe} / \mathrm{H}]$ derived in Valenti \& Fischer (2005). The coefficients, $a, b, c$, and $d$, together with the number of stars in common between Valenti \& Fischer (2005) and the original study are listed in Table 1.

These transformations were then used to move all entries in the 15 studies onto the common metallicity scale. We then used the General Catalogue of Photometric Data (Mermilliod et al. 1997) to find uvby photometry for these stars from the catalogues by Olsen and Schuster and collaborators. In total, 451 stars had $[\mathrm{Fe} / \mathrm{H}]$ derived from high-resolution spectroscopy and $u v b y$ photometry. As before, if a star had more than one set of uvby measurements the most recent was kept. The spectroscopic catalogue can be found in Table B.1.

Also for this catalogue we dereddened the photometry as described in Sect. 3.1. We recall that, the photometry for stars with $E(B-V)<0.02$ were not corrected. The implications of this are discussed in Sect. 4.1. Fifty stars in the catalogue have $E(B-V)>0.02$. The stellar distances are based on the reanalysed Hipparcos parallaxes (van Leeuwen 2007). Five stars HD 23261, HD 69582, HD 180890, HD 192020, and PLX 1219 do not have Hipparcos parallaxes. Their extinction was estimated using the method of Carney (1983) which is based on VJK photometry. These five stars do not have a Hipparcos number in Table B.1.

For two of the 15 studies, we note that no star redder than $(b-y)_{0}>0.6$ remained after the dereddening (see Table 1). These studies were nevertheless kept in the compilation as they provide valuable additional stars close to this border. Figure $4 \mathrm{~b}$ shows the distribution of the stars in the HR-diagram and Fig. 5 
Table 1. Coefficients for Eq. (2).

\begin{tabular}{lrrrrrrrr}
\hline \hline Study & Ref. & \# of stars & $\begin{array}{r}\text { \# of stars with } \\
(b-y)_{0}>0.6\end{array}$ & $a$ & $b$ & $c$ & $d$ & $\sigma$ \\
\hline Favata et al. (1997) & 2 & 46 & 1 & 1.0608 & -0.9662 & -0.7918 & 7.1781 & 0.07 \\
Feltzing \& Gustafsson (1998) & 3 & 23 & 2 & 0.7903 & -0.8958 & -0.1252 & 3.9841 & 0.05 \\
Chen et al. (2000) & 4 & 28 & 1 & 1.1759 & -2.0582 & -0.0767 & 8.2072 & 0.06 \\
Thorén \& Feltzing (2000) & 5 & 12 & 4 & 0.9918 & 0.0163 & -0.2020 & 0.8187 & 0.08 \\
Santos et al. (2001) & 6 & 61 & 1 & 1.0405 & -0.9088 & -0.0586 & 3.6431 & 0.04 \\
Heiter \& Luck (2003) & 7 & 75 & 0 & 0.8985 & 0.7027 & -0.1373 & -2.0303 & 0.05 \\
Yong \& Lambert (2003) & 8 & 6 & 2 & 1.1258 & 2.0980 & -0.2534 & -6.3255 & 0.05 \\
Mishenina et al. (2004) & 9 & 93 & 1 & 1.1434 & -1.8958 & -0.0888 & 7.5583 & 0.06 \\
Santos et al. (2004) & 10 & 141 & 1 & 1.0098 & -1.2361 & -0.0838 & 5.0008 & 0.04 \\
Bonfils et al. (2005) & 11 & 19 & 0 & 0.8761 & -0.5454 & -0.0422 & 2.2801 & 0.07 \\
Luck \& Heiter (2005) & 12 & 65 & 6 & 0.9736 & 0.7346 & -0.0249 & -2.6251 & 0.06 \\
Santos et al. (2005) & 13 & 64 & 7 & 1.0495 & -1.1400 & -0.0204 & 4.3450 & 0.04 \\
Woolf \& Wallerstein (2005) & 14 & 8 & 6 & 1.0192 & 0.0846 & -0.4226 & 1.5298 & 0.04 \\
Sousa et al. (2006) & 15 & 57 & 1 & 0.9360 & -0.9590 & -0.0805 & 3.9268 & 0.02 \\
\hline
\end{tabular}

Notes. Column 1 lists the study that is being moved onto the Valenti \& Fischer (2005) system and Col. 2 the reference number used in Table B.1. Column 3 lists the number of stars in common with Valenti \& Fischer (2005). These are used to obtain the coefficients. Column 4 lists the number of stars redder than $(b-y)_{0}=0.6$. Columns 5 to 8 list the coefficients used in Eq. (2), and Col. 9 lists the $\sigma$ for the difference between [Fe/H] in the study listed in Col. 1 and the $[\mathrm{Fe} / \mathrm{H}]$ derived once the data have been put on to the Valenti \& Fischer (2005) system. The difference is calculated in the sense $[\mathrm{Fe} / \mathrm{H}]_{\text {original }}$ minus $[\mathrm{Fe} / \mathrm{H}]_{\text {corrected }}$.
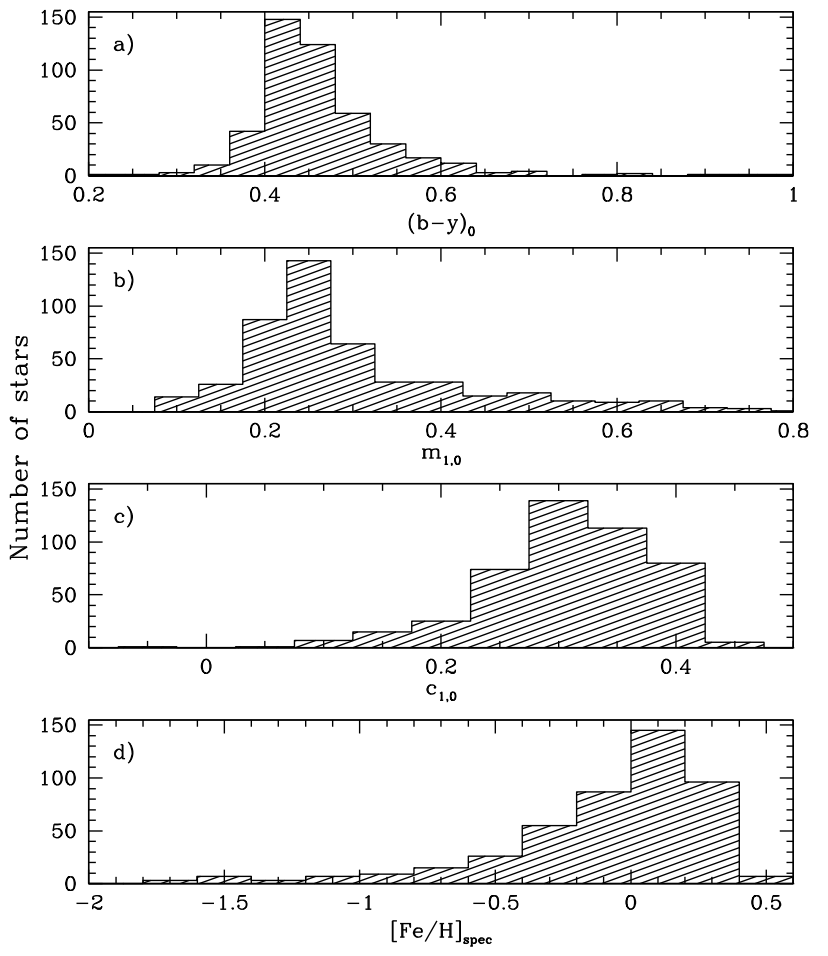

Fig. 5. Histograms showing the distribution of the photometric indices and $[\mathrm{Fe} / \mathrm{H}]$ for the spectroscopic catalogue (Table B.1 and Sect. 3.3). a) The number of stars as a function of $\left.(b-y)_{0}, \mathbf{b}\right)$ the number of stars as a function of $\left.m_{1,0}, \mathbf{c}\right)$ the number of stars as a function of $c_{1,0}$, and $\mathbf{d}$ ) the number of stars as a function of $[\mathrm{Fe} / \mathrm{H}]$.

shows the distributions of both the Strömgren indices and $[\mathrm{Fe} / \mathrm{H}]$ for the spectroscopic catalogue.

\section{Metallicities from uvby photometry - a critical evaluation}

The literature contains many calibrations that make it possible to derive metallicities from Strömgren photometry. Most of them are empirical but theoretical investigations also exist (see, e.g., Önehag et al. 2009, for a recent example). The early metallicity calibrations (Strömgren 1964; Crawford 1975; Olsen 1984) were mostly based on how much the colour indices $m_{1}$ and $c_{1}$ differed from a given standard relation, $\delta m_{1}=m_{1, \text { std }}-m_{1, \mathrm{obs}}$ and $\delta c_{1}=c_{1, \mathrm{obs}}-c_{1, \mathrm{std}}$. The $m_{1,0}-(b-y)_{0}$ and $c_{1,0}-$ $(b-y)_{0}$ relations used in these calibrations are usually derived from observations of stars belonging to the Hyades stellar cluster (for $\left.m_{1,0}-(b-y)_{0}\right)$ and from field stars that are believed to be on the ZAMS (for $\left.c_{1,0}-(b-y)_{0}\right)$. Olsen (1984) provides an example of how the preliminary standard sequences were derived.

More recent calibrations for dwarf stars have abandoned the use of standard relations (with the exception of Haywood 2002) and derive $[\mathrm{Fe} / \mathrm{H}]$ directly from the colour indices $(b-y)_{0}, m_{1,0}$, and $c_{1,0}$ (Schuster \& Nissen 1989b; Malyuto 1994; Haywood 2002; Martell \& Laughlin 2002; Martell \& Smith 2004; Nordström et al. 2004; Ramírez \& Meléndez 2005a; Holmberg et al. 2007). The metallicity calibration by Olsen (1984) is the only calibration that extends all the way to $(b-y)_{0}=1.0$. No calibration exists for dwarf stars redder than $(b-y)_{0}=1.0$.

In addition, some metallicity calibrations for dwarf stars require the use of the $\beta$ index (e.g., Nissen 1981; Twarog et al. 2007) or additional broadband photometry (Kotoneva et al. 2002; Bonfils et al. 2005; Flynn \& Morell 1997). These will be not be investigated here.

Already Bond (1980) found tentative evidence of a metallicity dependence in the Strömgren indices for red giant stars in the field, which was further investigated by Anthony-Twarog \& Twarog (1994), who also derived metallicity dependent standard sequences of red giants in the $c_{1,0}$ vs. $(b-y)_{0}$ diagram. Theoretical studies of the stellar colours of red giant stars found that the colours show clear dependencies on both metallicity and the amount of CNO in the atmospheres of the stars (Gustafsson \& Bell 1979). Hilker (2000) provided an updated calibration based on both field stars and red giant branch stars in globular clusters. However, the number of metallicity calibrations derived directly for red giant stars is limited. The list includes Bond (1980), Grebel \& Richtler (1992), Anthony-Twarog \& Twarog (1998), Hilker (2000), and Calamida et al. (2007). 
Table 2. Metallicity calibrations evaluated in Sect. 4.

\begin{tabular}{|c|c|c|c|c|c|c|}
\hline \multirow{2}{*}{ Reference } & \multicolumn{2}{|c|}{$(b-y)_{0}$} & \multicolumn{2}{|c|}{$[\mathrm{Fe} / \mathrm{H}]$} & \multirow{2}{*}{$\langle[\mathrm{Fe} / \mathrm{H}]-[\mathrm{M} / \mathrm{H}]\rangle \pm \sigma$} & \multirow[b]{2}{*}{ Comment } \\
\hline & $\min$ & $\max$ & $\min$ & $\max$ & & \\
\hline \multirow{3}{*}{ Olsen (1984) } & 0.29 & 1.00 & -2.60 & 0.39 & $0.11 \pm 0.34$ & Their Eq. (15) \\
\hline & 0.514 & 1.000 & -2.60 & 0.39 & $0.04 \pm 0.39$ & Their Eq. (15) \\
\hline & 0.514 & 1.000 & -0.25 & 0.60 & $0.02 \pm 0.17$ & Their Eq. (16) \\
\hline \multirow[t]{2}{*}{ Schuster \& Nissen (1989b) } & 0.22 & 0.38 & -3.5 & 0.2 & \multirow{2}{*}{$0.06 \pm 0.16$} & F-type dwarfs \\
\hline & 0.37 & 0.59 & -2.6 & 0.4 & & G-type dwarfs \\
\hline Haywood (2002) & 0.22 & 0.59 & -2.0 & 0.5 & $0.00 \pm 0.18$ & \\
\hline Martell \& Laughlin (2002) & 0.288 & 0.591 & -2.0 & 0.5 & $0.05 \pm 0.13$ & \\
\hline Martell \& Smith (2004) & 0.288 & 0.591 & -2.0 & 0.5 & $0.06 \pm 0.21$ & \\
\hline \multirow[t]{2}{*}{ Nordström et al. (2004) } & 0.18 & 0.38 & -2.0 & 0.8 & $-0.17 \pm 0.52$ & \\
\hline & 0.44 & 0.59 & -2.0 & 0.8 & $0.13 \pm 0.08$ & \multirow{4}{*}{$\begin{array}{l}\text { F-type dwarfs } \\
\text { G-type dwarfs }\end{array}$} \\
\hline \multirow[t]{2}{*}{ Ramírez \& Meléndez (2005a) } & 0.19 & 0.35 & -3.5 & 0.4 & \multirow{2}{*}{$0.04 \pm 0.14$} & \\
\hline & 0.35 & 0.80 & -2.5 & 0.4 & & \\
\hline Holmberg et al. (2007) & 0.24 & 0.63 & -1.00 & 0.37 & $0.08 \pm 0.16$ & \\
\hline Önehag et al. (2008) & 0.22 & 0.59 & -3.5 & 0.4 & $0.33 \pm 0.30$ & \\
\hline
\end{tabular}

Notes. Column 1 lists the reference for the calibration. In Cols. 2 to 5 we quote the ranges, for for $(b-y)_{0}$ and $[\mathrm{Fe} / \mathrm{H}]$, within which the calibrations is valid. Column 6 gives the mean difference between $[\mathrm{Fe} / \mathrm{H}]$ and $[\mathrm{M} / \mathrm{H}]$ and the associated $\sigma$. Column 7 provides additional comments.

\subsection{A test of metallicity calibrations for dwarf stars}

We now use our compilation of dwarf stars in Table B.1 to evaluate how well various metallicity calibrations can reproduce $[\mathrm{Fe} / \mathrm{H}]$. We investigate the calibrations by Olsen (1984), Schuster \& Nissen (1989b), Haywood (2002), Martell \& Laughlin (2002), Martell \& Smith (2004), Nordström et al. (2004), Ramírez \& Meléndez (2005a), and Holmberg et al. (2007). The common aspect of these calibrations is that they are relatively recent and/or have been influential. In Sect. 6.2, we discuss the ability of model atmospheres to reproduce the observed Strömgren indices (Önehag et al. 2009).

We note that there are two metallicity calibrations in Olsen (1984). Both calibrations depend on $\delta m_{1}$, but while Eq. (16) in Olsen (1984) is a linear equation in $\delta m_{1}$, Eq. (15) includes a quadratic term in $\delta m_{1}$. We investigate both calibrations.

Each calibration was applied only to stars with photometric indices in the range where the calibration is valid (as indicated in the original study). In Table 2, we list the mean difference between $[\mathrm{Fe} / \mathrm{H}]$ and $[\mathrm{M} / \mathrm{H}]$. As can be seen, the mean offset is, in most of the cases, smaller than 0.1 dex. Two calibrations yield larger offsets, Olsen (1984) (full range of Eq. (15)) and Nordström et al. (2004). These calibrations also have some of the largest scatters (compare Table 2 and Figs. 6 and 7).

Figure 6 compares the differences between $[\mathrm{Fe} / \mathrm{H}]$ and $[\mathrm{M} / \mathrm{H}]$ as a function of $[\mathrm{Fe} / \mathrm{H}]$. There is a tendency for some of the calibrations (notably Schuster \& Nissen 1989b; Martell \& Smith 2004; Holmberg et al. 2007) to show a declining trend towards lower $[\mathrm{Fe} / \mathrm{H}]$. The second and third of these calibrations also show obvious trends with $(b-y)_{0}$ when $(b-y)_{0} \geq 0.5$. Hence, even if these calibrations formally extend all the way to about 0.6 , it is clear that there are shortcomings for the redder colours.

A comparison of the difference as a function of $m_{1,0}$ (Fig. 7) indicates that two of the calibrations (Martell \& Smith 2004; Holmberg et al. 2007) fall short at the redder end of the distribution. Finally, studying the difference as function of $c_{1,0}$ we note that Holmberg et al. (2007) appears to show some real trend for the lower $c_{1,0}$ and that Martell \& Smith (2004), and possibly Haywood (2002), show an overall trend such that the metallicity is underestimated at low $c_{1,0}$ and overestimated at high $c_{1,0}$.

In summary, we find that both Schuster \& Nissen (1989b) and Ramírez \& Meléndez (2005a) perform very well in all four comparisons. However, as Ramírez \& Meléndez (2005a) covers a much larger parameter space we would recommend it over Schuster \& Nissen (1989b), but again recall that in the regions where the two calibrations overlap they perform equally well.

However, Ramírez \& Meléndez (2005a) extends only to $(b-y)_{0}=0.8$. We therefore investigated the redder calibration of Olsen (1984). In Fig. 8, we compare the $[\mathrm{Fe} / \mathrm{H}]$ with the resulting $[\mathrm{M} / \mathrm{H}]$ from that calibration, finding good agreement. In Fig. 9, we compare the results from Olsen (1984) with the results from Ramírez \& Meléndez (2005a) as a function of $(b-y)_{0}$, and again find close agreement. From these tests, we conclude that Olsen (1984) provides an adequate extension of Ramírez \& Meléndez (2005a) for stars redder than $(b-y)_{0}=0.8$.

As discussed in Sect. 3.1, if the reddening towards a star is less than 0.02 we do not apply a reddening correction (Table B.1). The effect of this omission is small. For example, if we use the calibration of Ramírez \& Meléndez (2005a) to calculate $[\mathrm{M} / \mathrm{H}]$ and assume that stars with $E(B-V)<0.02$, have an $E(B-V)=0.02$ the mean difference between $[\mathrm{Fe} / \mathrm{H}]$ and $[\mathrm{M} / \mathrm{H}]$ changes from $0.041 \pm 0.140$ to $-0.003 \pm 0.148$. The trends with $[\mathrm{Fe} / \mathrm{H}]$ and the photometric indices change very little. To the eye, it appears that, e.g., for redder $(b-y)_{0}$ the scatter increases. Similar trends are seen for the other indices.

\subsection{Metallicity calibrations for red giant branch stars}

Faria et al. (2007) undertook a detailed investigation of the calibrations then available and found that the calibration of Hilker (2000) was by far the most successful when comparing with high-resolution spectroscopy. However, Faria et al. (2007) only gives a limited comparison of metal-poor, faint red giant stars in the Draco dwarf spheroidal galaxy. Ramírez \& Meléndez (2004) undertook a comparison with field giants in the Milky Way ranging from solar all the way down to -2.5 dex. They found that the Hilker (2000) calibration underestimated the intermediate metallicities but overestimated the lowest metallicities when compared to the spectroscopically derived iron abundances. Solar metallicities were well reproduced. Ramírez \& Meléndez (2004) provide a correction formula to place the calibrations of Hilker (2000) onto the spectroscopic scale. Since then, Calamida et al. (2007) presented a new, and very comprehensive, study of metallicities of red giant stars and their iron abundance. This study 

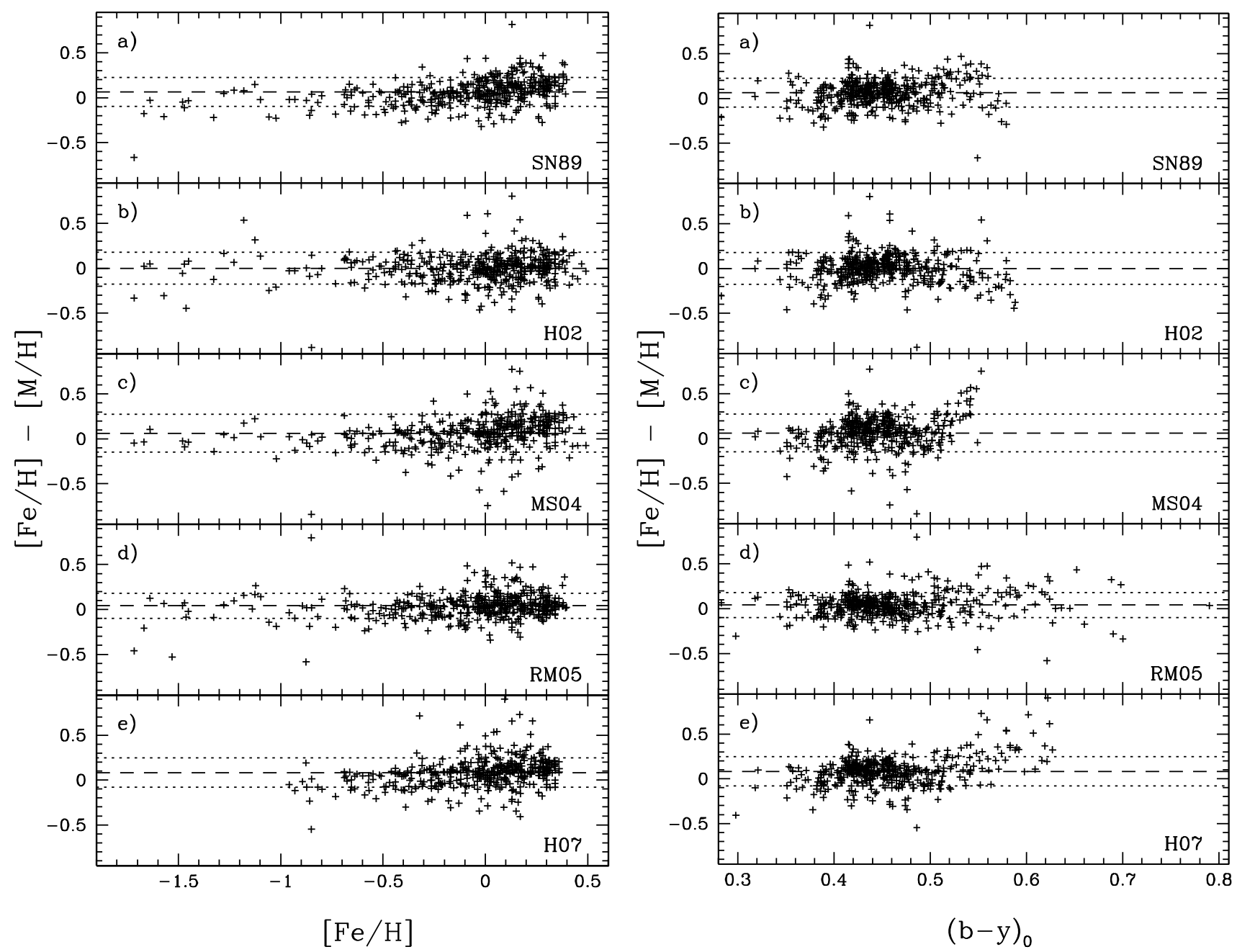

Fig. 6. The difference between $[\mathrm{Fe} / \mathrm{H}]$ and $[\mathrm{M} / \mathrm{H}]$, derived from the calibrations listed in Table 2, as a function of $[\mathrm{Fe} / \mathrm{H}]($ left hand panels) and $(b-y)_{0}$ (right hand panels). The metallicity calibrations used are labelled as follows: SN89 for Schuster \& Nissen (1989b), MS04 for Martell \& Smith (2004), H07 for Holmberg et al. (2007), H02 for Haywood (2002), and RM05 for Ramírez \& Meléndez (2005a). The mean differences (dashed lines) and the $\sigma$ (dotted lines) are listed in Table 2.

used giant stars in globular clusters as a reference for their calibration. Calamida et al. (2007) used the more metallicity sensitive index $(v-y)_{0}$, rather than $(b-y)_{0}$ used in Hilker (2000). As discussed already by Strömgren (1963), the position of the $v$ filter provides a measure of the total decrement due to the presence of metallicity lines. We refer the reader to Calamida et al. (2007) and Calamida et al. (2009) (which provides an update to Calamida et al. 2007) for an extended discussion of the derivation of their metallicity calibration for red giant stars.

Figure 10 compares the different calibrations applied to metal-poor red giant branch stars in three nearby dwarf spheroidal galaxies (Draco, Sextans, and Hercules). For this comparison, we use the calibration by Calamida et al. (2007) as reference. Data for Draco and Sextans are taken from Adén et al. (in prep.) and data for Hercules from Adén et al. (2009a). The data in Adén et al. (in prep.) will supersede those of Faria et al. (2007).

The comparison between Calamida et al. (2007) and Hilker (2000) shows the same banana shape noted by Ramírez \& Meléndez (2004). This is most prominently seen for stars in the Draco dwarf spheroidal galaxy. The difference between Calamida et al. (2007) and Calamida et al. (2009) is, as expected, very small, the major difference being at the most metal-poor end. Comparing Calamida et al. (2007) and the corrected Hilker (2000) calibration by Ramírez \& Meléndez (2004) indicates that the calibration by Ramírez \& Meléndez (2004) would produce a more metal-poor as well as more concentrated metallicity distribution function for the three galaxies than if we used the calibration by Calamida et al. (2007). Calamida et al. (2009) use $(v-y)$ and $(u-v)$ for their calibrations; although these colours are more sensitive to metallicity than $(b-y)$ they are also sensitive to $\mathrm{CH}$ and CN. It appears, however, from the comparison carried out here, that the choice of colours to use in the calibration might not be very sensitive to the presence of molecules (at least for the giant stars in the dwarf spheroidal galaxies). This should, however, be further studied.

We note that all of these calibrations are poorly constrained at the metal-poor end and more calibration data are required to improve the calibrations. Many studies currently target stars in the metal-poor dwarf spheroidal galaxies and these data will thus become available soon. We also note that to date only the calibration by Ramírez \& Meléndez (2004) extends to solar metallicity, which is an important property for investigations where more metal-rich stars can be expected.

Calibrations of $u v b y$ photometry for red giant stars with metallicities below -2 dex have not been rigorously tested 
A. S. Árnadóttir et al.: Metallicities and stellar classification from Strömgren photometry
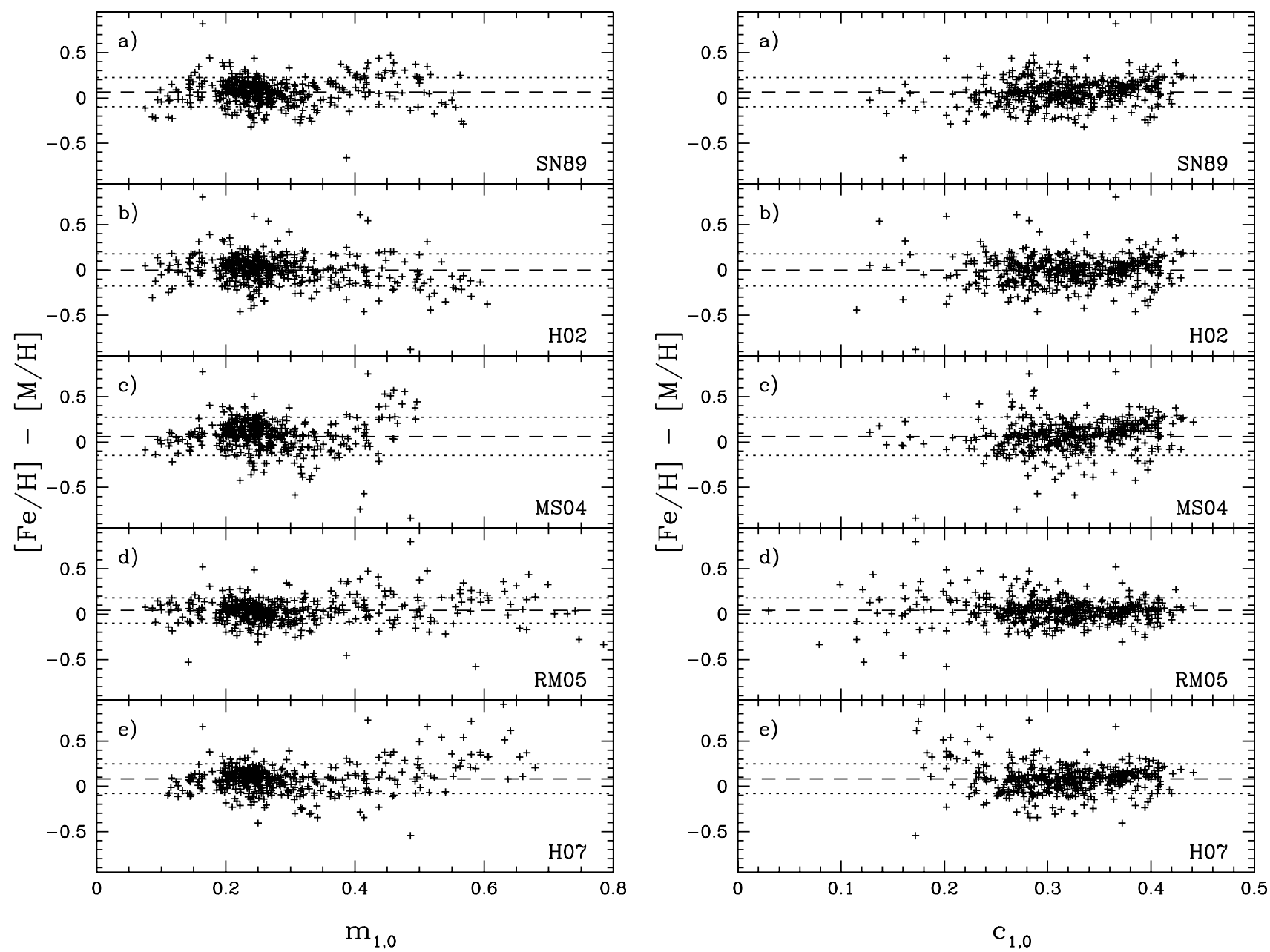

Fig. 7. The difference between $[\mathrm{Fe} / \mathrm{H}]$ and $[\mathrm{M} / \mathrm{H}]$, derived from the calibrations listed in Table 2, as a function of $m_{1,0}$ (left-hand panels) and $c_{1,0}$ (right-hand panels). The metallicity calibrations used are labelled as follows: SN89 for Schuster \& Nissen (1989b), MS04 for Martell \& Smith (2004), H07 for Holmberg et al. (2007), H02 for Haywood (2002), and RM05 for Ramírez \& Meléndez (2005a). The mean differences (dashed lines) and the $\sigma$ (dotted lines) are listed in Table 2.

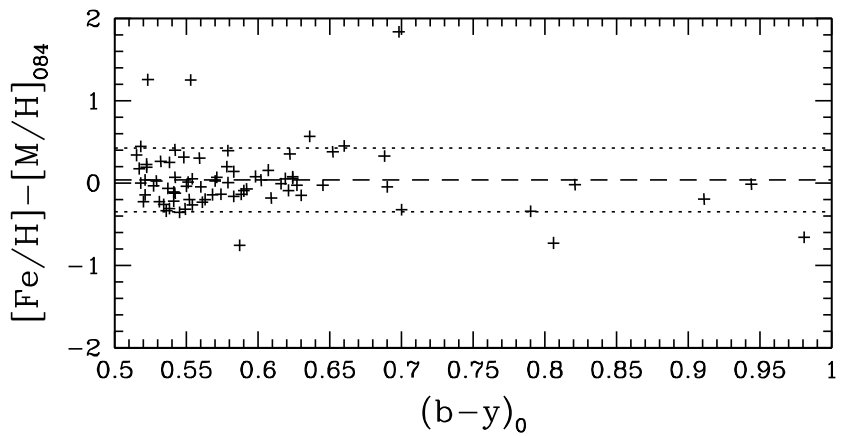

Fig. 8. The difference between $[\mathrm{Fe} / \mathrm{H}]$ and $[\mathrm{M} / \mathrm{H}]$ calculated using the calibration by Olsen $(1984)\left([\mathrm{M} / \mathrm{H}]_{\mathrm{O} 84}\right)$. The comparison is made in the colour interval $0.514<(b-y)_{0}<1.000$. The mean difference is 0.03 dex (dashed line) with a $\sigma$ of 0.39 dex (dotted lines).

because uvby photometry and iron abundances based on highresolution spectroscopy for metal-poor field red giant have been largely unavailable. However, a first look at data for Hercules (Adén et al., submitted) indicates that $[\mathrm{Fe} / \mathrm{H}]$ based on high resolution spectroscopy for about ten red giant branch stars infers lower iron abundances than predicted from photometry using

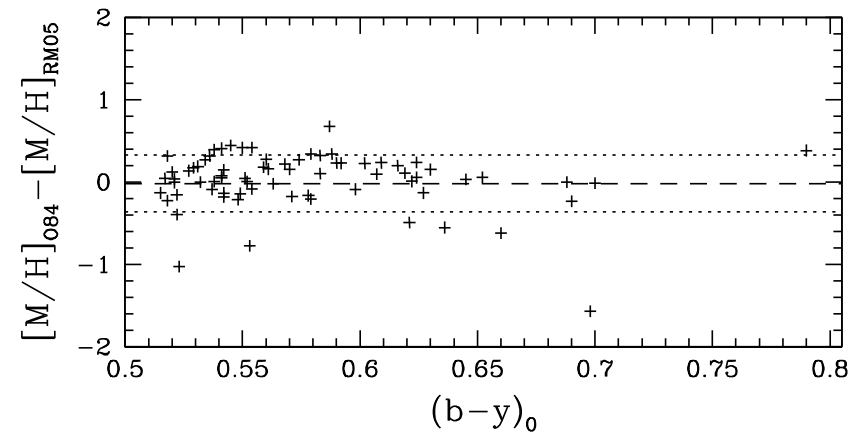

Fig. 9. A comparison of the $[\mathrm{M} / \mathrm{H}]$ calculated using the calibration by Olsen (1984) $\left([\mathrm{M} / \mathrm{H}]_{\mathrm{O} 84}\right)$ and the calibration by Ramírez \& Meléndez (2005a) $\left([\mathrm{M} / \mathrm{H}]_{\mathrm{RM} 05}\right)$. The comparison is made in the colour interval $0.514<(b-y)_{0}<0.800$. The mean difference is -0.02 dex (dashed line) with a $\sigma$ of 0.39 dex (dotted lines).

any of the metallicity calibrations discussed here. In addition, preliminary comparisons with data from Kirby et al. (2008) find the same result (Adén et al. 2009a; and Adén et al., submitted). This conclusion is supported by a comparison with the new Draco data by Cohen \& Huang (2009), who obtained highresolution spectroscopy of eight of the brighter red giants in 


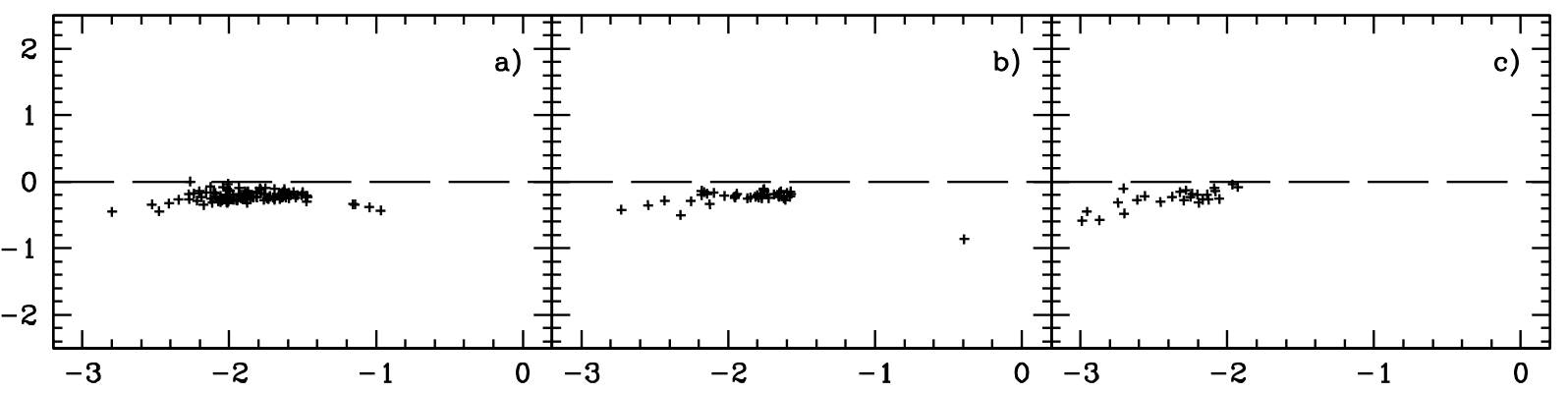

$[\mathrm{M} / \mathrm{H}]_{\text {uvby,C07 }}$
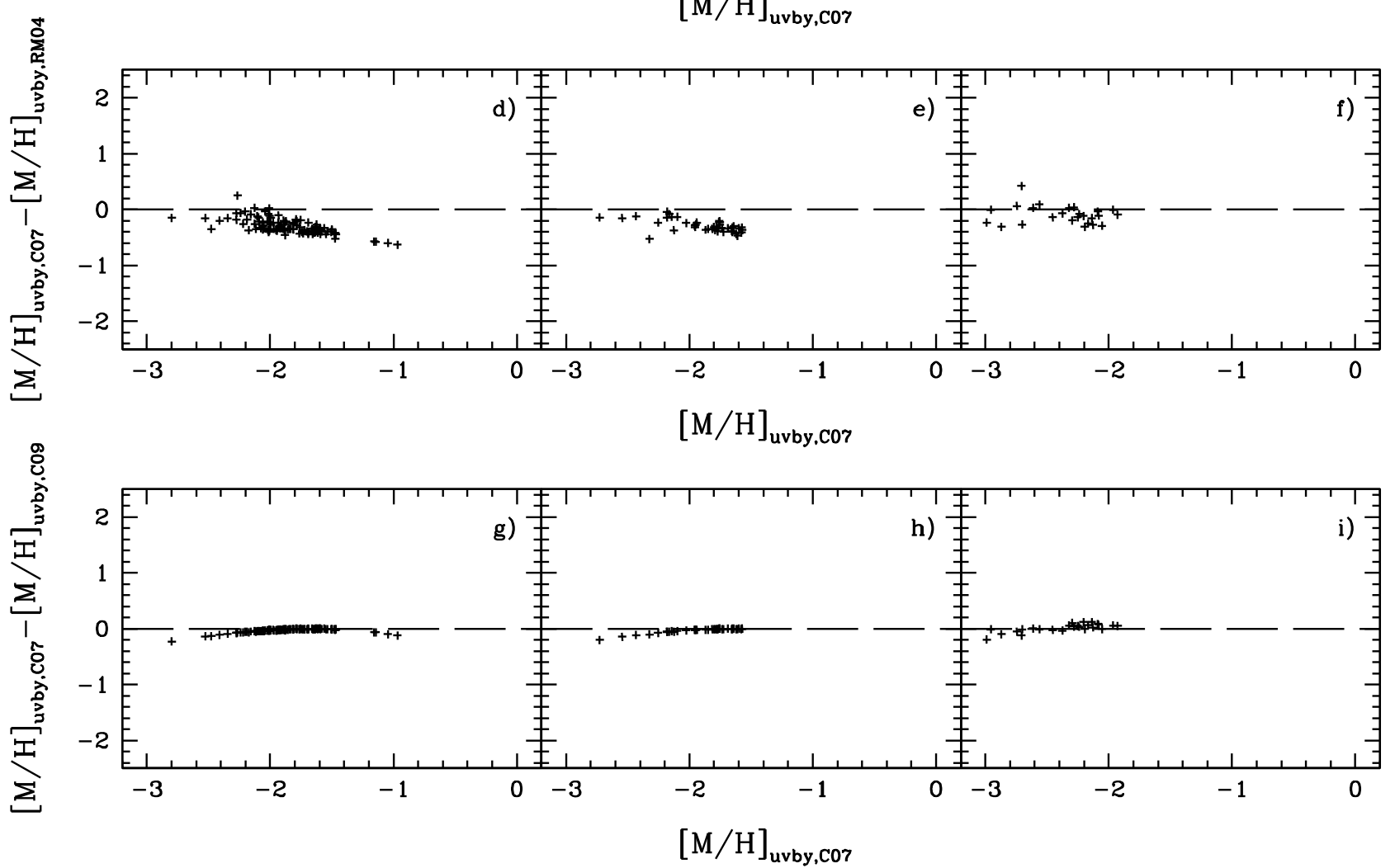

Fig. 10. A comparison of $[\mathrm{M} / \mathrm{H}]$ derived for giant stars using the four most recent metallicity calibrations for uvby photometry. We use the calibration of Calamida et al. (2007) as the reference for all comparisons. Panels a), d), and g) shows the data for stars in the Draco dwarf spheroidal galaxy, panels b), e), and h) the data for stars in the Sextans dwarf spheroidal galaxy, and panels c), f), and i) data for giant stars in the Hercules dwarf spheroidal galaxy.

the Draco dwarf spheroidal. We have Strömgren photometry for six of these stars. A comparison with $[\mathrm{M} / \mathrm{H}]$ derived using the calibration of Calamida et al. (2009) gives a mean difference of -0.21 dex and a $\sigma$ of 0.19 dex. A similar comparison but using the calibration by Ramírez \& Meléndez (2004) gives a mean difference of -0.25 dex and a $\sigma$ of 0.22 dex. Cohen \& Huang (2009) noted a similar difference when they compared their spectroscopic $[\mathrm{Fe} / \mathrm{H}]$ with those metallicities derived using the calibration of Hilker (2000). We note that the most metalpoor stars in the sample cause the largest deviations. Above about -2 dex, the comparison is very favourable. As part of our ongoing work on uvby photometry for red giant stars in dwarf spheroidal galaxies, we are evaluating the possibilities to extend current metallicity calibrations for uvby photometry to metallicities below -2 dex.

We also compared the iron abundances of giant stars in the Draco dwarf spheroidal galaxy determined in Cohen \& Huang (2009) with metallicities derived from ugriz photometry using the calibration of Ivezić et al. (2008). The scatter is very large and some of the metallicities are clearly incorrect. The differences are such that even with a very large sample and considering, e.g., only the mean metallicity of the sample the conclusions would be at best indicative (see also Sect. 4.3 below).

\subsection{A comparison with photometric metallicities from SDSS - both dwarf and giant stars}

The SDSS (York et al. 2000) is one of the most influential studies covering a very large portion of the sky. The stellar part contains not only ugriz photometry but also spectra for a large fraction of the objects. This and additional spectroscopic campaigns provide $[\mathrm{M} / \mathrm{H}]$ (e.g., Lee et al. 2008). It is of great interest to attempt to derive calibrations to use the ugriz photometry to provide stellar parameters and in particular [M/H] (Ivezić et al. 2008). If good calibrations can be obtained, much new information about 

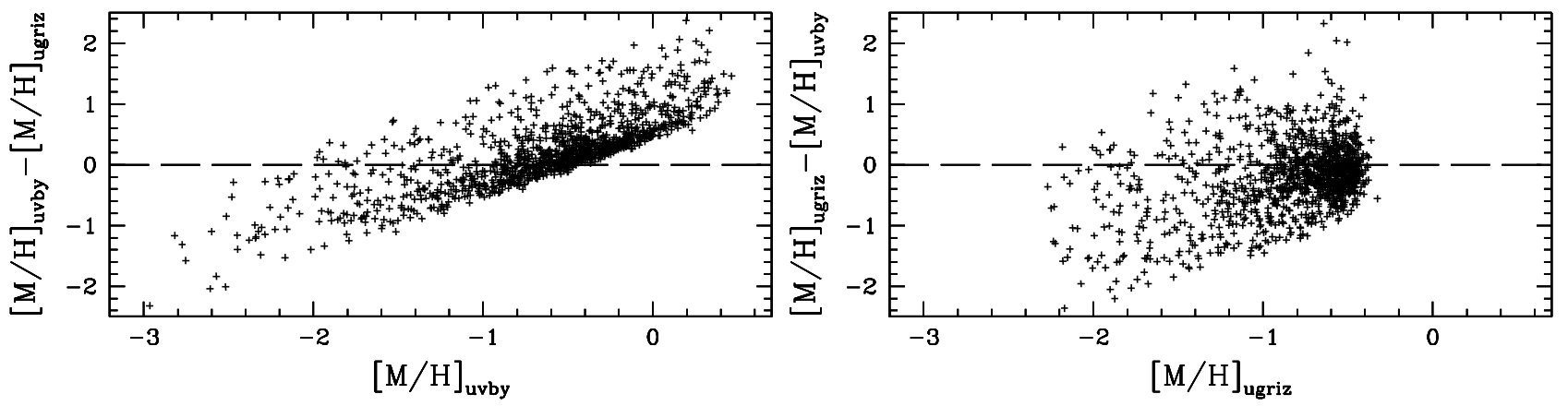

Fig. 11. A comparison of metallicities for dwarf stars derived from $u v b y$ photometry $\left([\mathrm{M} / \mathrm{H}]_{\text {uvby }}\right)$ using the calibrations by Ramírez \& Meléndez (2005a) and Olsen (1984) and metallicities derived from SDSS ugriz photometry ([M/H $]_{\text {ugriz }}$ ) using the calibration of Ivezić et al. (2008). The stars are along the lines-of-sight in the directions of the Hercules, Draco, and Sextans dwarf spheroidal galaxies. A full description of how these stars were selected will be provided in Árnadóttir et al. (in prep.). All stars have $15<V_{0}<18.5$. The dashed line indicates a metallicity difference of zero. On the abscissa the left-hand panel has $[\mathrm{M} / \mathrm{H}]_{\text {wby }}$ and the right-hand panel has $[\mathrm{M} / \mathrm{H}]_{\text {ugriz }}$.

the thick disk and the halo can be obtained (see, e.g., Carollo et al. 2008). Because of the potential impact of SDSS, it remains important to test the calibrations against independent metallicity measures. Our Strömgren photometry provides an opportunity to do so for a large sample of fairly faint dwarf and giant stars.

To perform these comparisons we use uvby photometry of dwarf stars from Árnadóttir et al. (in preparation) and data for red giant stars from Faria (2006), Faria et al. (2007), Lagerholm (2008), Adén et al. (2009a), and Adén et al. (in prep.). The identification of dwarfs and giants is unambiguous for the stars we use (see e.g., Faria et al. 2007; Adén et al. 2009a). The ugriz photometry is from SDSS DR7 (Abazajian et al. 2009).

For the uvby photometry, we use the calibrations of Ramírez \& Meléndez (2005a) and Olsen (1984) (for dwarf stars) and Calamida et al. (2007) (for giant stars) to calculate $[\mathrm{M} / \mathrm{H}]_{\text {uvby }}$. For the ugriz photometry, we use the calibration of Ivezic et al. (2008) to calculate $[\mathrm{M} / \mathrm{H}]_{\text {ugriz }}$. The comparisons between $[\mathrm{M} / \mathrm{H}]_{\text {uvby }}$ and $[\mathrm{M} / \mathrm{H}]_{\text {ugriz }}$ are shown in Figs. 11 and 12.

We first note that for the dwarf stars in Fig. 11 there is good agreement at metallicities around -1 dex, but that agreement quickly deteriorates as we move to higher or lower metallicities. There is some scatter but there is a distinctive linear relation such that $[\mathrm{M} / \mathrm{H}]_{\text {ugriz }}$ is higher than $[\mathrm{M} / \mathrm{H}]_{\text {uvby }}$ at low metallicities and the opposite is true for solar metallicities. At solar metallicity, the offset is about $0.5 \mathrm{dex}$ and at $[\mathrm{M} / \mathrm{H}]_{u v b y}=-2$ the offset is about 1.5 dex. Given the fairly extensive tests that have been performed to compare $[\mathrm{M} / \mathrm{H}]_{u v b y}$ to $[\mathrm{Fe} / \mathrm{H}]$ derived from stellar spectroscopy provided both in this study (see Sect. 4 and Figs. 6 and 7) and elsewhere, these differences are a concern.

A comparison for metallicities for giants presented in Fig. 12 is perhaps even less encouraging. For $-3<[\mathrm{M} / \mathrm{H}]_{\text {uvby }}<-2$, there is a trend similar to that for the dwarf stars, but at higher metallicities the relation appears to break down completely. We note that our datasets for the giant stars are small but we believe that the more populated red giant branch of the Draco dwarf spheroidal galaxy provides a fairly unambiguous result. It is beyond the scope of this paper to explain these differences. However, given the very large discrepancies in some cases caution is required when using $[\mathrm{M} / \mathrm{H}]_{\text {ugriz }}$ to infer the properties of the halo, where clearly many of the targets will be giants. Given the overall scatter for giant stars of metallicity -2 dex, a typical halo metallicity, in Fig. 12 these inferences must be regarded as only indicative.

The comparison between $[\mathrm{M} / \mathrm{H}]_{u v b y}$ and $[\mathrm{Fe} / \mathrm{H}]$ from high resolution spectroscopy indicates that $[\mathrm{M} / \mathrm{H}]_{u v b y}$ is overestimated
(Sect. 4.2). If $[\mathrm{M} / \mathrm{H}]_{\text {uvby }}$ were corrected to more closely match $[\mathrm{Fe} / \mathrm{H}]$, then the difference between $[\mathrm{M} / \mathrm{H}]_{\text {ugriz }}$ and $[\mathrm{M} / \mathrm{H}]_{\text {uvby }}$ would be even greater.

\section{The uvby system's ability to distinguish between dwarf, sub-giant, and giant stars - New stellar sequences}

The Strömgren $u v b y$ system has a proven ability to distinguish between dwarf and giant stars for certain colour ranges. We have used this in two studies of dwarf spheroidal galaxies to remove the foreground contamination by Milky Way dwarf stars (Faria et al. 2007; Adén et al. 2009a). In the most recent paper, we showed that about $30 \%$ of the stars that would otherwise be assumed to be radial velocity members of the Hercules dwarf spheroidal galaxy are instead foreground dwarf stars. This result has lead to a re-evaluation of the minimum common mass for such galaxies (compare, e.g., Strigari et al. 2008; Adén et al. 2009b).

A significant drawback is that the stellar sequences merge around $(b-y)_{0}=0.55$ in the $c_{1,0}$ vs. $(b-y)_{0}$ diagram. For bluer colours, the lower red giant branch almost meets the main sequence and the subgiant branch and turn-off forms a loop (see Fig. 2). Twarog et al. (2007) investigated whether a new index could be developed to distinguish between dwarf, sub-giant, and giant stars at bluer colours. We also performed fairly extensive tests with our datasets described in Sect. 3 based on our studies of dwarf spheroidal galaxies (Faria et al. 2007; Adén et al. 2009a); we found that for larger datasets the proposed new index does not appear to have the desired ability to distinguish between the bluer dwarf, sub-giant, and giant stars.

\subsection{Metallicity-dependent dwarf star sequences}

Dwarf star sequences in the Strömgren $c_{1,0}-\left(b-y_{0}\right)$ plane were introduced for F-type dwarf stars by Crawford (1975) and later extended to $(b-y)=1.0$ by Olsen (1984). These sequences were drawn by hand tracing the lower envelope of field stars in the relevant diagram. No attempts were made to investigate if the stellar sequences were metallicity dependent, although this possibility was discussed already by Strömgren (1964). It is clear, in the $c_{1,0}$ vs. ( $\left.b-y_{0}\right)$ diagram, when we compare the dwarf star sequence of Olsen (1984) to the dwarf region for metal-poor stars, given by Schuster et al. (2004), that the metal-poor dwarf stars have lower $c_{1,0}$ indices than the, mainly, solar metallicity stars 

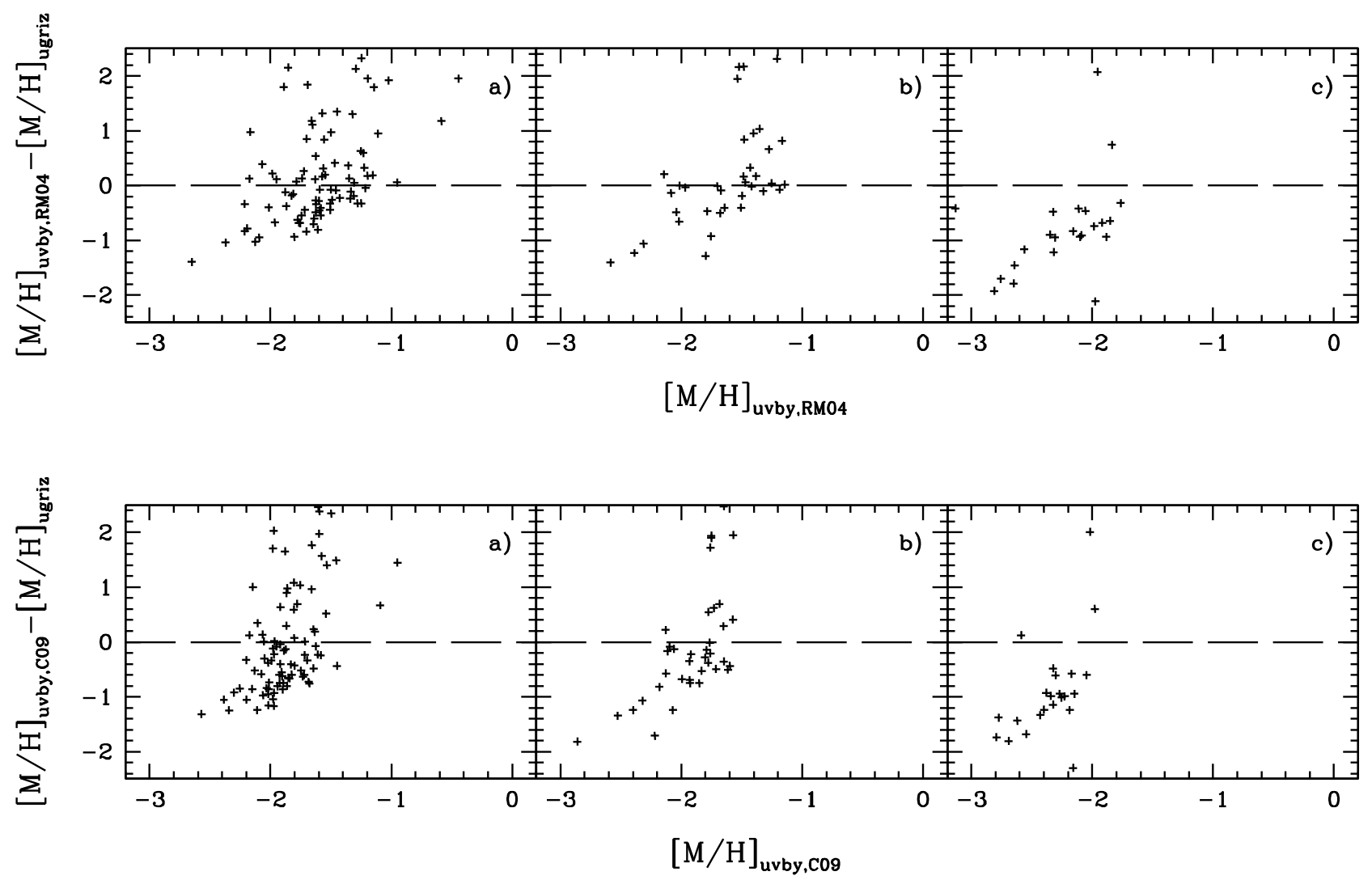

Fig. 12. A comparison of metallicities derived from uvby photometry and ugriz photometry (using the calibration of Ivezić et al. 2008), respectively, for giant stars in dwarf spheroidal galaxies. The top panels uses the calibration by Ramírez \& Meléndez (2004) and the bottom panels the calibration by Calamida et al. (2009) to obtain metallicities from uvby photometry. a) Comparison for red giant branch stars in the Draco dwarf spheroidal galaxy (uvby photometry: Adén et al., in prep.). b) Comparison for red giant branch stars in the Sextans dwarf spheroidal galaxy (uvby photometry: Adén et al., in prep.; and Lagerholm 2008). c) Comparison for red giant branch stars in the Hercules dwarf spheroidal galaxy (vby photometry: Adén et al. 2009a).

used to define the sequence in Olsen (1984). This can be seen, e.g., in Fig. 2.

We are now in a position to extend the study of Olsen (1984) and investigate the metallicity dependence of dwarf star sequences in both the $c_{1,0}$ vs. $(b-y)_{0}$ diagram and the $c_{1,0}$ vs. $(v-y)_{0}$ diagram. For stars in our photometric catalogue $[\mathrm{M} / \mathrm{H}]$ were calculated (see Sect. 3.2) using the metallicity calibrations by Ramírez \& Meléndez (2005a) for dwarf and subgiant stars with $(b-y)_{0}<0.80$ and the calibration by Olsen (1984) for dwarf stars with $0.80<(b-y)_{0}<1.00$.

To trace the stellar (standard) sequences, we plotted $c_{1,0}$ vs. $(b-y)_{0}$ and $c_{1,0}$ vs. $(v-y)_{0}$ for the dwarf stars, but each time only for a narrow range in metallicity. Following the procedure in Olsen (1984), we trace the lower envelope of the stellar distribution in both the $c_{1,0}$ vs. $(b-y)_{0}$ and $c_{1,0}$ vs. $(v-y)_{0}$ diagrams. This lower envelope is sensitive to metallicity. For $(b-y)_{0}>0.7$, all dwarf stars fall on a tight relation without any dependence on metallicity. We used all stars redder than $(b-y)_{0} \sim 0.7$ to define the sequence up to $(b-y)_{0}=1.0$. Our data set has no stars redder than 1.0. Figure 13 shows two examples of how these tracings were done. Figures A.1 to A.14 in Appendix A show all tracings. The sequences are tabulated in Tables 3 and 4.

Although we have extended the tracings to as blue colours as possible in Figs. A.1 to A.14, it is clear that for colours bluer than $(b-y)_{0}=0.4$ the data are not substantial enough in quantity at any metallicity to provide a secure tracing. Moreover, we use only stars classified as GKV in Olsen (1993), Olsen (1994a), and Olsen (1994b), and therefore exclude bluer main sequence stars. This exclusion is also colour dependent because it depends on the metallicity of the stars. Because of these limitations we refrain from listing the tracings bluer than $(b-y)_{0}=0.4$ and $(v-y)_{0}=1.1$.

We also traced a global upper envelope for all dwarf stars. This upper envelope is listed in Table 5.

\subsection{The ability of the ugriz photometric system to identify giant stars}

Helmi et al. (2003)used ugriz photometry to identify metal-poor giant stars. We test this method using stars in the direction of the Draco dwarf spheroidal galaxy. The field contains both foreground dwarf stars in the Milky Way as well as metal-poor giant stars in the dwarf spheroidal galaxy (Faria 2006; Faria et al. 2007; Árnadóttir et al., in prep.; Adén et al., in prep.).

Helmi et al. (2003) define a new colour index, $s=-0.249 u+$ $0.794 g-0.555 r+0.24$ which is used to identify the metal-poor giant stars. They find that metal-poor giant stars in general have larger $s$-indices than the dwarf stars and define a giant star as a star with an $s$-index more than 0.05 mag above the median $s$-index for the field.

We use metal-poor giant stars in the Draco dwarf spheroidal galaxy and foreground stars along the same line-of-sight to test the ability of the $s$-index to distinguish dwarf from giant stars. The ugriz colour-magnitude diagram for the field used is shown in Fig. 14a. For the comparison, we only use stars in the colour 
A. S. Árnadóttir et al.: Metallicities and stellar classification from Strömgren photometry
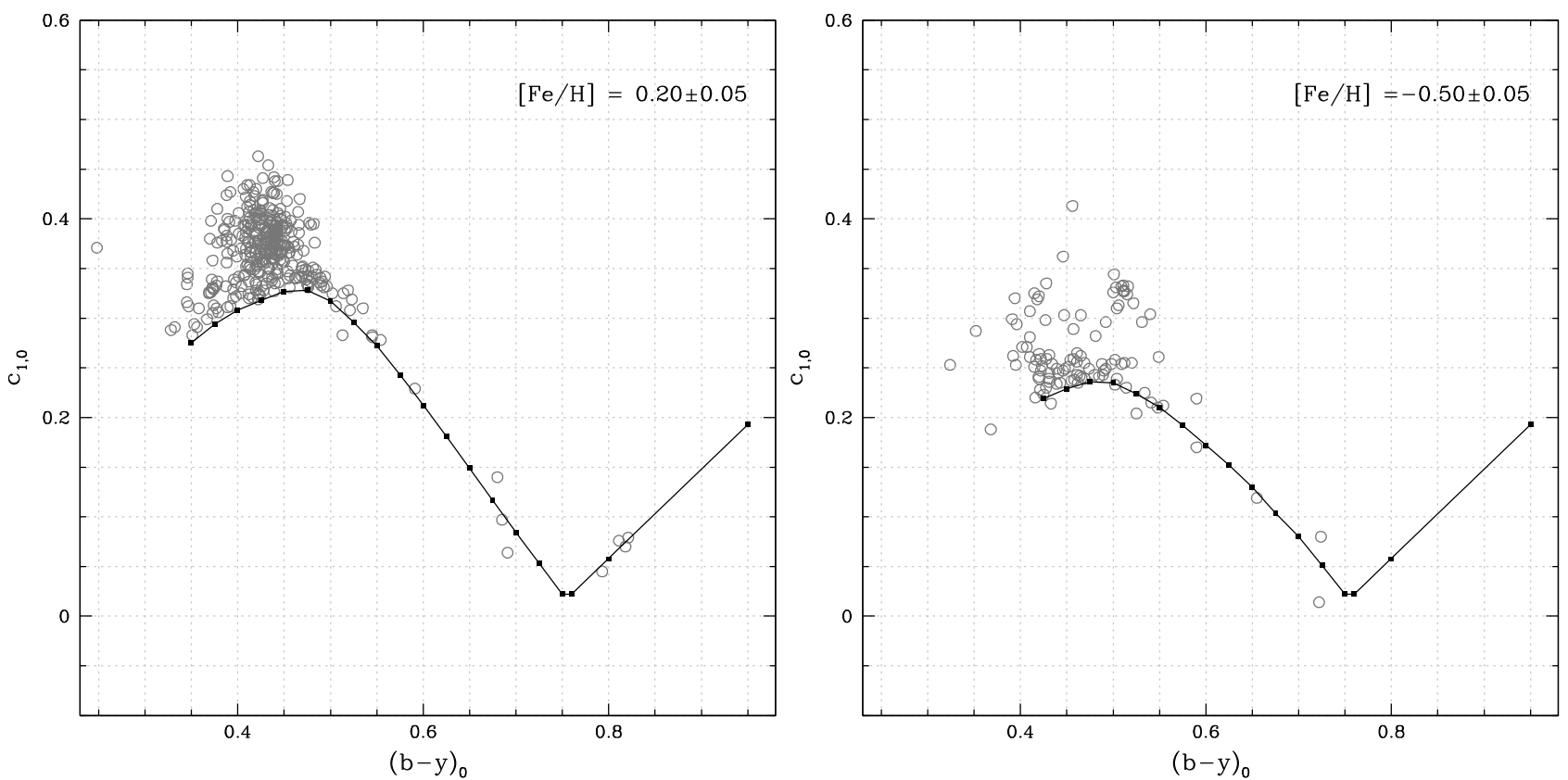

Fig. 13. Two examples of how the dwarf sequences in the $c_{1}$ vs. $(b-y)$ diagram, discussed in Sect. 5.1, were established. The left hand panel shows dwarf stars with $0.15<[\mathrm{M} / \mathrm{H}]<0.25$ and the right hand panel dwarf stars with $-0.55<[\mathrm{M} / \mathrm{H}]<-0.45$. A complete set of similar plots for all metallicities can be found in Appendix A (available online). The standard relations are listed in Tables 3 and 4.

Table 3. New metallicity-dependent sequences for dwarf stars (see Sect. 5.1 and Figs. 13, and A.1 to A.14). For each range of metallicity (as indicated in the top two rows), we list the $c_{1,0}$ value for each $(b-y)_{0}$, as listed in the first column.

\begin{tabular}{|c|c|c|c|c|c|c|c|c|c|c|c|c|c|c|}
\hline $\begin{array}{l}{[\mathrm{M} / \mathrm{H}]} \\
\pm\end{array}$ & $\begin{array}{l}0.50 \\
0.05\end{array}$ & $\begin{array}{l}0.40 \\
0.05\end{array}$ & $\begin{array}{l}0.30 \\
0.05\end{array}$ & $\begin{array}{l}0.20 \\
0.05\end{array}$ & $\begin{array}{l}0.10 \\
0.05\end{array}$ & $\begin{array}{l}0.00 \\
0.05\end{array}$ & $\begin{array}{r}-0.10 \\
0.05\end{array}$ & $\begin{array}{r}-0.20 \\
0.05\end{array}$ & $\begin{array}{r}-0.30 \\
0.05\end{array}$ & $\begin{array}{r}-0.40 \\
0.05\end{array}$ & $\begin{array}{r}-0.50 \\
0.05\end{array}$ & $\begin{array}{r}-0.60 \\
0.10\end{array}$ & $\begin{array}{r}-0.80 \\
0.15\end{array}$ & $\begin{array}{r}-1.00 \\
0.20\end{array}$ \\
\hline$(b-y)_{0}$ & $c_{1,0}$ & $c_{1,0}$ & $\overline{c_{1,0}}$ & $c_{1,0}$ & $c_{1,0}$ & $c_{1,0}$ & $c_{1,0}$ & $c_{1,0}$ & $c_{1,0}$ & $c_{1,0}$ & $c_{1,0}$ & $c_{1,0}$ & $c_{1,0}$ & $c_{1,0}$ \\
\hline 0.400 & 0.380 & 0.345 & 0.326 & 0.308 & 0.288 & 0.271 & 0.252 & 0.244 & 0.242 & 0.228 & - & 0.217 & - & - \\
\hline 0.425 & 0.398 & 0.363 & 0.338 & 0.318 & 0.300 & 0.284 & 0.265 & 0.252 & 0.242 & 0.229 & 0.219 & 0.211 & 0.192 & 0.140 \\
\hline 0.450 & 0.395 & 0.378 & 0.347 & 0.327 & 0.310 & 0.293 & 0.278 & 0.267 & 0.249 & 0.238 & 0.229 & 0.217 & 0.194 & 0.146 \\
\hline 0.475 & 0.371 & 0.371 & 0.347 & 0.328 & 0.310 & 0.298 & 0.285 & 0.274 & 0.256 & 0.247 & 0.236 & 0.227 & 0.196 & 0.154 \\
\hline 0.500 & 0.341 & 0.341 & 0.336 & 0.317 & 0.305 & 0.292 & 0.280 & 0.272 & 0.259 & 0.249 & 0.235 & 0.228 & 0.196 & 0.161 \\
\hline 0.525 & 0.309 & 0.309 & 0.307 & 0.296 & 0.290 & 0.276 & 0.266 & 0.262 & 0.254 & 0.243 & 0.224 & 0.220 & 0.193 & 0.165 \\
\hline 0.550 & 0.277 & 0.277 & 0.276 & 0.272 & 0.267 & 0.258 & 0.245 & 0.243 & 0.238 & 0.230 & 0.210 & 0.207 & 0.186 & 0.165 \\
\hline 0.575 & 0.245 & 0.245 & 0.245 & 0.243 & 0.240 & 0.235 & 0.223 & 0.221 & 0.219 & 0.211 & 0.192 & 0.190 & 0.175 & 0.163 \\
\hline 0.600 & 0.213 & 0.213 & 0.213 & 0.212 & 0.210 & 0.209 & 0.198 & 0.196 & 0.195 & 0.186 & 0.172 & 0.172 & 0.162 & 0.153 \\
\hline 0.625 & 0.181 & 0.181 & 0.181 & 0.181 & 0.180 & 0.180 & 0.173 & 0.171 & 0.171 & 0.162 & 0.152 & 0.152 & 0.145 & 0.139 \\
\hline 0.650 & 0.149 & 0.149 & 0.149 & 0.149 & 0.149 & 0.149 & 0.145 & 0.144 & 0.144 & 0.136 & 0.130 & 0.130 & 0.126 & 0.120 \\
\hline 0.675 & 0.117 & 0.117 & 0.117 & 0.117 & 0.117 & 0.117 & 0.115 & 0.115 & 0.115 & 0.109 & 0.104 & 0.104 & 0.103 & 0.097 \\
\hline 0.700 & 0.084 & 0.084 & 0.084 & 0.084 & 0.084 & 0.084 & 0.084 & 0.084 & 0.084 & 0.082 & 0.080 & 0.080 & 0.077 & 0.072 \\
\hline 0.725 & 0.053 & 0.053 & 0.053 & 0.053 & 0.053 & 0.053 & 0.053 & 0.053 & 0.053 & 0.052 & 0.051 & 0.051 & 0.049 & 0.047 \\
\hline 0.750 & 0.022 & 0.022 & 0.022 & 0.022 & 0.022 & 0.022 & 0.022 & 0.022 & 0.022 & 0.022 & 0.022 & 0.022 & 0.022 & 0.022 \\
\hline 0.760 & 0.022 & 0.022 & 0.022 & 0.022 & 0.022 & 0.022 & 0.022 & 0.022 & 0.022 & 0.022 & 0.022 & 0.022 & 0.022 & 0.022 \\
\hline 0.800 & 0.058 & 0.058 & 0.058 & 0.058 & 0.058 & 0.058 & 0.058 & 0.058 & 0.058 & 0.058 & 0.058 & 0.058 & 0.058 & 0.058 \\
\hline 0.950 & 0.193 & 0.193 & 0.193 & 0.193 & 0.193 & 0.193 & 0.193 & 0.193 & 0.193 & 0.193 & 0.193 & 0.193 & 0.193 & 0.193 \\
\hline
\end{tabular}

range $1.1<(u-g)<2.0$ and $0.3<(g-r)<0.8$, where the $s$-index is defined (Helmi et al. 2003). We identify metal-poor giant stars in the direction of the Draco dwarf spheroidal galaxy with $16.0<V_{0}<19.2$ using the Strömgren $c_{1,0}-(b-y)_{0}$ diagram (see Fig. 14c). In Fig. 14c, the dwarf region is indicated as a shaded region (Árnadóttir et al., in prep.).

Figure $14 \mathrm{~d}$ shows the $V_{0}-s$ diagram for stars selected as dwarf and giant stars using the Strömgren $c_{1,0}-(b-y)_{0}$ diagram. The metal-poor giant stars that we identify in the $c_{1,0}$ vs. $(b-y)_{0}$ plane have a wide range of $s$-index values. The dotted line indicates the $s$-value above which metal-poor giant stars should be found. Figure 14d shows that metal-poor giant stars can not be distinguished from the dwarf stars using the $s$-index. Although the stars identified using the $s$-index are predominantly metal-poor giant stars, the $s$-index is unable to reliably differentiate between metal-poor giant stars and the foreground dwarf stars to good accuracy. More importantly, the majority of the giant stars can not be identified by the $s$-index.

\section{A comparison of stellar sequences and model predictions}

The stellar sequences for dwarf stars constructed in Sect. 5.1 can be compared with model predictions based on stellar evolutionary tracks and stellar model atmospheres. Such comparisons are important for two reasons, they help us to 
Table 4. New metallicity-dependent sequences for dwarf stars (see Sect. 5.1). For each range of metallicity (as indicated in the top two rows), we list the $c_{1,0}$ value for each $(v-y)_{0}$, as listed in the first column.

\begin{tabular}{|c|c|c|c|c|c|c|c|c|c|c|c|c|c|c|}
\hline$[\mathrm{M} / \mathrm{H}]$ & 0.50 & 0.40 & 0.30 & 0.20 & 0.10 & 0.00 & -0.10 & -0.20 & -0.30 & -0.40 & -0.50 & -0.60 & -0.80 & -1.00 \\
\hline \pm & 0.05 & 0.05 & 0.05 & 0.05 & 0.05 & 0.05 & 0.05 & 0.05 & 0.05 & 0.05 & 0.05 & 0.10 & 0.15 & 0.20 \\
\hline$(v-y)_{0}$ & $c_{1,0}$ & $\overline{c_{1,0}}$ & $\overline{c_{1,0}}$ & $c_{1,0}$ & $c_{1,0}$ & $c_{1,0}$ & $c_{1,0}$ & $c_{1,0}$ & $c_{1,0}$ & $c_{1,0}$ & $c_{1,0}$ & $c_{1,0}$ & $c_{1,0}$ & $c_{1,0}$ \\
\hline 1.100 & 0.381 & 0.336 & 0.321 & 0.308 & 0.292 & 0.273 & 0.260 & 0.254 & 0.245 & 0.231 & 0.226 & 0.211 & 0.190 & 0.140 \\
\hline 1.150 & 0.393 & 0.349 & 0.332 & 0.315 & 0.299 & 0.281 & 0.271 & 0.260 & 0.247 & 0.237 & 0.230 & 0.215 & 0.195 & 0.147 \\
\hline 1.200 & 0.400 & 0.359 & 0.339 & 0.322 & 0.306 & 0.289 & 0.278 & 0.265 & 0.253 & 0.241 & 0.233 & 0.219 & 0.198 & 0.152 \\
\hline 1.250 & 0.395 & 0.365 & 0.342 & 0.327 & 0.309 & 0.294 & 0.283 & 0.271 & 0.257 & 0.245 & 0.236 & 0.227 & 0.201 & 0.156 \\
\hline 1.300 & 385 & 0.367 & 0.345 & 0.328 & 0.310 & 0.297 & 0.285 & 0.274 & 0.260 & 0.247 & 0.237 & 0.233 & 0.202 & 0.159 \\
\hline 1.350 & 375 & 0.364 & 0.345 & 0.326 & 0.308 & 0.296 & 0.284 & 0.272 & 0.261 & 0.248 & 0.237 & 0.234 & 0.203 & 0.162 \\
\hline 1.400 & 0.363 & 0.358 & 0.340 & 0.322 & 0.305 & 0.294 & 0.282 & 0.271 & 0.260 & 0.247 & 0.236 & 0.232 & 0.202 & 0.164 \\
\hline 1.450 & 0.350 & 0.348 & 0.332 & 0.314 & 0.298 & 0.288 & 0.276 & 0.266 & 0.255 & 0.246 & 0.232 & 0.228 & 0.200 & 0.166 \\
\hline 1.500 & 0.337 & 0.335 & 0.323 & 0.304 & 0.288 & 0.281 & 0.268 & 0.259 & 0.249 & 0.243 & 0.225 & 0.222 & 0.196 & 0.166 \\
\hline 1.550 & 0.324 & 0.322 & 0.312 & 0.293 & 0.279 & 0.271 & 0.260 & 0.251 & 0.242 & 0.238 & 0.219 & 0.215 & 0.192 & 0.165 \\
\hline 1.600 & 0.309 & 0.308 & 0.301 & 0.282 & 0.268 & 0.261 & 0.250 & 0.242 & 0.232 & 0.229 & 0.211 & 0.207 & 0.186 & 0.163 \\
\hline 1.650 & 0.296 & 0.294 & 0.289 & 0.270 & 0.257 & 0.250 & 0.239 & 0.232 & 0.221 & 0.219 & 0.202 & 0.198 & 0.177 & 0.161 \\
\hline 1.700 & 0.279 & 0.278 & 0.275 & 0.258 & 0.245 & 0.239 & 0.227 & 0.221 & 0.208 & 0.207 & 0.192 & 0.188 & 0.168 & 0.155 \\
\hline 1.750 & 0.264 & 0.263 & 0.261 & 0.245 & 0.232 & 0.227 & 0.215 & 0.210 & 0.195 & 0.194 & 0.181 & 0.177 & 0.159 & 0.148 \\
\hline 1.800 & 0.246 & 0.246 & 0.245 & 0.231 & 0.218 & 0.214 & 0.202 & 0.198 & 0.183 & 0.181 & 0.170 & 0.166 & 0.150 & 0.140 \\
\hline 1.850 & 0.229 & 0.229 & 0.228 & 0.215 & 0.203 & 0.200 & 0.188 & 0.185 & 0.170 & 0.168 & 0.158 & 0.154 & 0.140 & 0.130 \\
\hline 1.900 & 0.211 & 0.211 & 0.210 & 0.198 & 0.187 & 0.185 & 0.174 & 0.172 & 0.157 & 0.154 & 0.146 & 0.141 & 0.129 & 0.120 \\
\hline 1.950 & 0.191 & 0.191 & 0.190 & 0.181 & 0.171 & 0.169 & 0.159 & 0.158 & 0.143 & 0.140 & 0.133 & 0.128 & 0.118 & 0.110 \\
\hline 2.000 & 0.169 & 0.169 & 0.169 & 0.162 & 0.154 & 0.152 & 0.143 & 0.142 & 0.129 & 0.126 & 0.120 & 0.115 & 0.107 & 0.099 \\
\hline 2.050 & 0.146 & 0.146 & 0.146 & 0.142 & 0.136 & 0.135 & 0.126 & 0.125 & 0.116 & 0.111 & 0.106 & 0.102 & 0.095 & 0.088 \\
\hline 2.100 & 0.122 & 0.122 & 0.122 & 0.120 & 0.116 & 0.115 & 0.108 & 0.107 & 0.101 & 0.095 & 0.091 & 0.087 & 0.083 & 0.076 \\
\hline 2.150 & 0.097 & 0.098 & 0.098 & 0.097 & 0.097 & 0.094 & 0.090 & 0.089 & 0.085 & 0.080 & 0.077 & 0.074 & 0.070 & 0.064 \\
\hline 2.200 & 0.074 & 0.074 & 0.074 & 0.073 & 0.073 & 0.072 & 0.070 & 0.069 & 0.067 & 0.063 & 0.061 & 0.059 & 0.056 & 0.051 \\
\hline 2.250 & 0.050 & 0.050 & 0.050 & 0.050 & 0.050 & 0.050 & 0.049 & 0.049 & 0.048 & 0.044 & 0.042 & 0.041 & 0.040 & 0.037 \\
\hline 2.310 & 0.022 & 0.022 & 0.022 & 0.022 & 0.022 & 0.022 & 0.022 & 0.022 & 0.022 & 0.022 & 0.022 & 0.022 & 0.022 & 0.022 \\
\hline 2.350 & .120 & 0.120 & 0.120 & 0.120 & 0.120 & 0.120 & 0.120 & 0.120 & 0.120 & 0.120 & 0.120 & 0.120 & 0.120 & 0.120 \\
\hline 2.380 & 0.200 & 0.200 & 0.200 & 0.200 & 0.200 & 0.200 & 0.200 & 0.200 & 0.200 & 0.200 & 0.200 & 0.200 & 0.200 & 0.200 \\
\hline
\end{tabular}

Table 5. The upper envelope for dwarf stars in the solar neighbourhood.

\begin{tabular}{lrr}
\hline \hline$c_{1,0}$ & $(b-y)_{0}$ & $(v-y)_{0}$ \\
\hline 0.396 & 0.350 & 0.895 \\
0.423 & 0.375 & 0.947 \\
0.448 & 0.400 & 1.010 \\
0.458 & 0.410 & 1.044 \\
0.461 & 0.430 & 1.085 \\
0.450 & 0.450 & 1.131 \\
0.424 & 0.470 & 1.205 \\
0.385 & 0.490 & 1.305 \\
\hline
\end{tabular}

understand the physical processes occurring inside stars (stellar evolution) and the processes in the stellar photospheres (e.g., how well we can model the lines in the resulting stellar spectra). Additionally, after ensuring that we understand these processes (to a certain level), we may utilise the resulting stellar isochrones and theoretically calculate indices to infer, e.g., the age of a globular cluster.

In Fig. 15, we compare our new stellar sequences for dwarf stars with the preliminary relations of Olsen (1984) and Crawford (1975). As can be seen, the metallicity dependence is significant and the lower envelope changes by about 0.1 in $c_{1}$ as we change the metallicity with 0.5 dex. For the reddest part, we agree with the preliminary sequences in that there is only a single relation (see discussion in Sect. 5.1), although the slopes of the sequences differ.

\subsection{A comparison with stellar isochrones}

Few isochrones have been calculated for the Strömgren photometric system, the most important set is probably that provided by Vandenberg \& Bell (1985) and derivations from that work. To convert theoretical stellar evolutionary sequences into stellar isochrones, a colour-temperature relation is required (e.g., Lester et al. 1986; Clem et al. 2004). The empirical calibration of Clem et al. (2004) is the most recent and is used to convert, e.g., the isochrones of Vandenberg \& Bell (1985) and their derivatives onto the observed plane. Clem et al. (2004) performed a detailed comparison between stellar isochrones produced using their colour-temperature relation and sequences of, e.g., red giant branches for globular clusters with different metallicities, finding a good agreement.

Faria et al. (2007) preformed an additional comparison of the stellar isochrones produced using the colour-temperature relation by Clem et al. (2004) with uvby photometry for field stars for which $[\mathrm{Fe} / \mathrm{H}]$ had been determined by high-resolution spectroscopy. Their dataset is essentially identical to that used by Clem et al. (2004) to obtain the, interpolated, colour-temperature relation for metallicities between -2 dex and super-solar metallicities. The comparison found some (still) unexplained discrepancies between the data and the isochrones. However, it was confirmed that the isochrones for about -2 dex and solar metallicity fit the field stars, with those metallicities, very well. Hence, there might be some problems with the empirical calibration needed for the colour transformation at intermediate metallicities. Here, we therefore repeat the comparison, this time as a comparison between our stellar sequences for dwarf stars and the isochrones derived using the colour-temperature relation by Clem et al. (2004). 
A. S. Árnadóttir et al.: Metallicities and stellar classification from Strömgren photometry
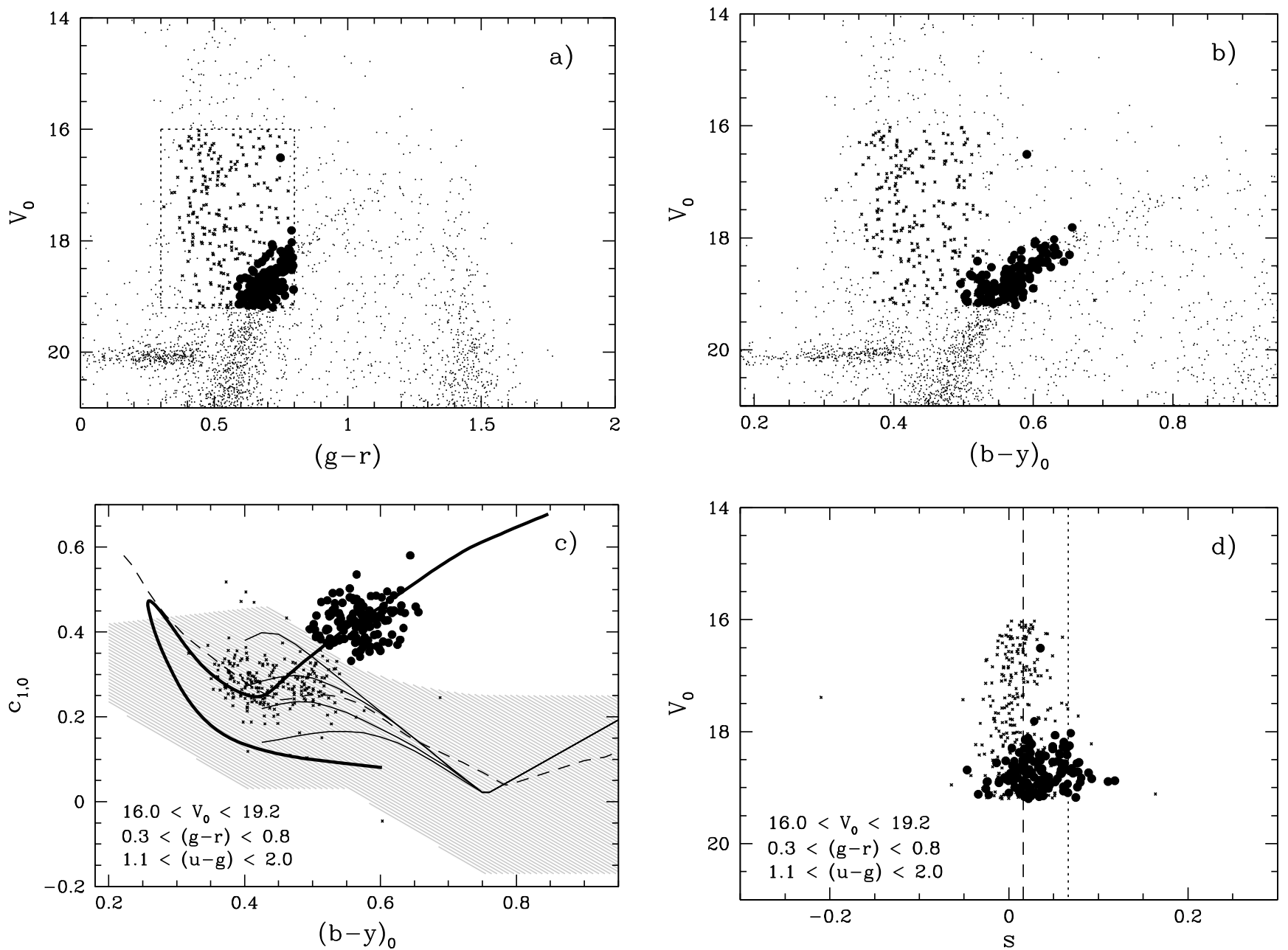

Fig. 14. a) Colour-magnitude diagram showing the selection of stars along the line of sight towards the Draco dSph galaxy used for testing the giant star identification of Helmi et al. (2003). These have $16.0<V_{0}<19.2,1.1<(u-g)<2.0$ and $0.3<(g-r)<0.8$ (marked with a box). Stars identified as giant stars in the $c_{1,0}$ vs. $(b-y)_{0}$ plane are shown as filled dots. b) The same stars but in a colour-magnitude diagram based on Strömgren photometry. Same symbols as in panel a). The box indicated by a dotted line in a) is not included as it is a non-square area once mapped into this colour-magnitude plane. c) Identification of giant stars (filled dots) in the $c_{1,0}$ vs. $(b-y)_{0}$ plane. Grey hashed area shows the dwarf region used in Árnadóttir et al. (in prep.). Our new dwarf star sequences (solid lines) are shown along with the preliminary relations by Olsen (1984) and Crawford (1975) (dashed line), and an isochrone with an age of $12 \mathrm{Gyr}$ and $[\mathrm{Fe} / \mathrm{H}]=-2.3$ (thick solid line, Vandenberg \& Bell 1985; Clem et al. 2004). d) The distribution of identified giant stars (filled dots) in the $s$-index of Helmi et al. (2003). Dashed line indicates the median $s$ of the selected stars (0.016) and the dotted line indicates the limit above which metal-poor giant stars are identified according to Helmi et al. (2003).

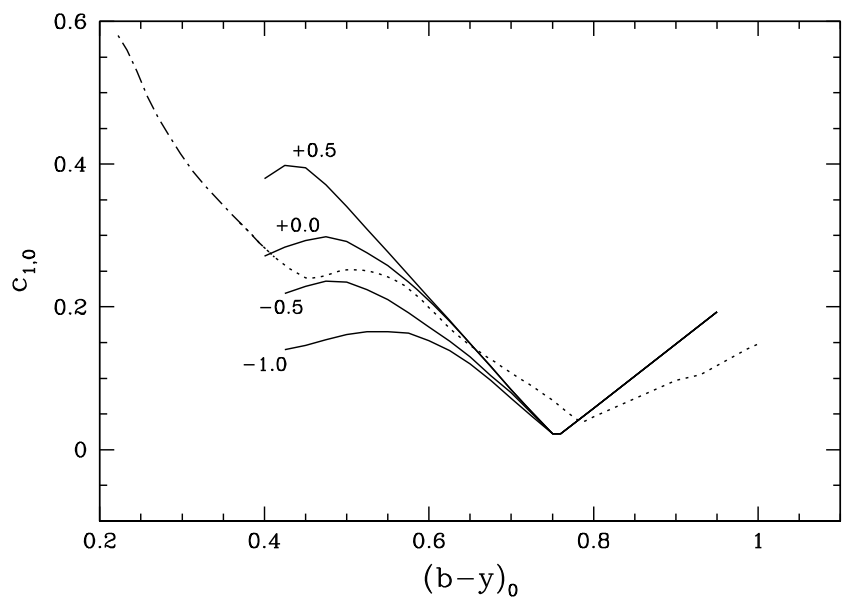

Fig. 15. Comparison of the new dwarf star sequences (solid lines), metallicities as indicated, to the preliminary relations by Olsen (1984) (dotted line) and by Crawford (1975) (dot-dashed line).
The comparison is shown in Fig. 16. The stellar sequences and the isochrones in general agree well with our sequences for dwarf stars at $0.45<(b-y)_{0}<0.7$. We note, however, that the stellar sequences trace the lower envelope of all stars that have a narrow range of metallicities (see Table 3 ) and the isochrones should reproduce the mean metallicity. Hence, there might be some offset with respect to the $c_{1,0}$ index, but otherwise the agreement is good for this fairly narrow magnitude range of dwarf stars. This comparison spans the main sequence from the turn-off, late F-type dwarf stars to three magnitudes down the main sequence to $M_{V} \sim 8$ (compare with Fig. $4 \mathrm{~b}$ ).

We performed a comparison between our sequences for dwarf stars, the stellar isochrones, and the calculated indices in the $c_{1,0}$ vs. $(b-y)_{0}$ diagram. This makes for an easy comparison with earlier works that often used $(b-y)_{0}$ as the colour along the $x$-axis. However, the $(v-y)_{0}$ colour is more sensitive to metallicity, as shown, e.g., by Calamida et al. (2007). This is true for both giant and dwarf stars. Although the $(v-y)_{0}$ is more sensitive to metallicity than $(b-y)_{0}$, it has the disadvantage that is is also 


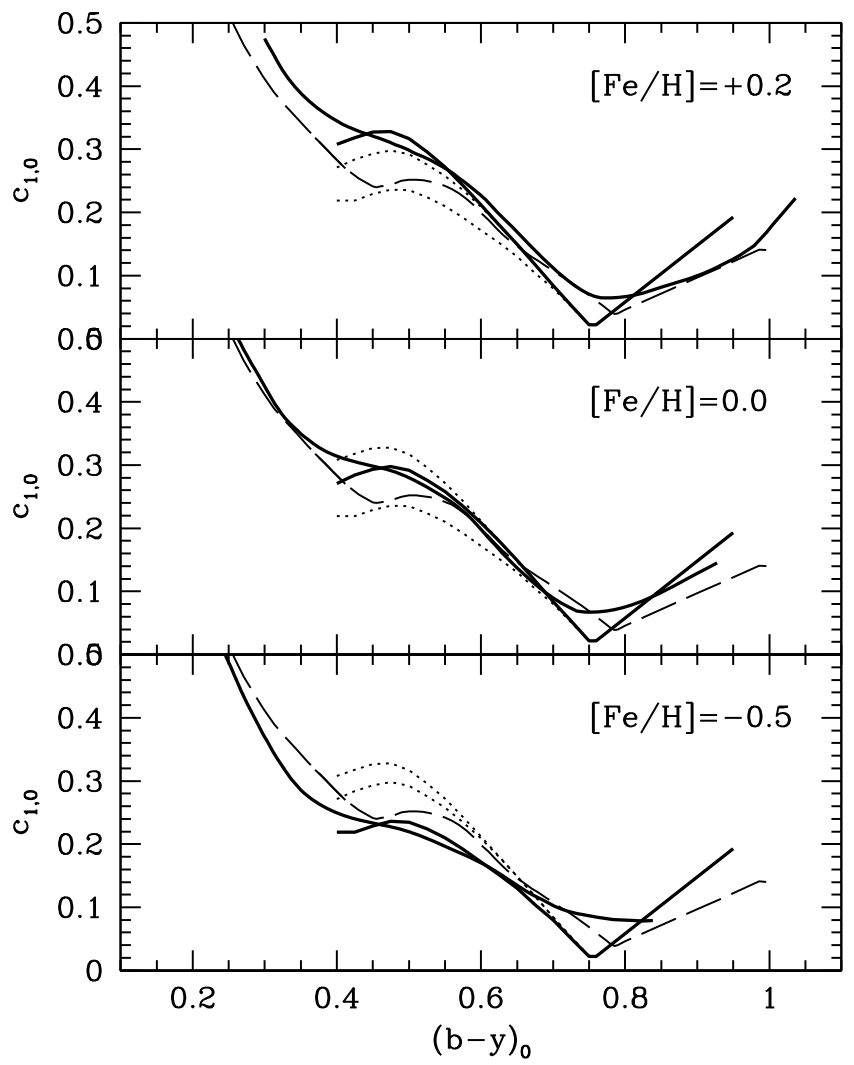

Fig. 16. A comparison of dwarf star sequences, as derived in this paper with stellar isochrones. In each panel, we show three of our sequences for dwarf stars for $[\mathrm{Fe} / \mathrm{H}]=+0.20,0.0$, and -0.5 . In each panel, the sequence with the metallicity indicated in the panel is shown with a thick solid line, the other two sequences are shown with dotted lines. The preliminary sequences by Olsen (1984) and by Crawford (1975) are shown with long dashes. An isochrone with the correct metallicity is also shown in each panel (thin, solid line). These isochrones are indicated with thick lines and all have an age of 1 Gyr (Vandenberg \& Bell 1985; Clem et al. 2004).

sensitive to the presence of $\mathrm{CH}$ and $\mathrm{CN}$ molecules in the stellar atmosphere.

\subsection{A note about calculated indices}

Theoretical indices in the Strömgren system have been studied in several articles, including Lester et al. (1986), Gustafsson \& Bell (1979), and Önehag et al. (2009). In Fig. 17, we perform a nonexhaustive comparison between our stellar sequences for dwarf stars and indices calculated by Önehag et al. (2009) for stars with $\log g=4.5$. We show stellar sequences for 0 and $-0.5 \mathrm{dex}$ because we believe that the sequence for -1 dex is less robust (compare Fig. A.14). It is clear from this comparison that the calculated indices do not reproduce the colours found for field dwarf stars in the solar neighbourhood for $(b-y)_{0}>0.45$.

Based on the calculated indices, Önehag et al. (2009) derive a metallicity calibration that is nominally valid for stars with $0.22<(b-y)_{0}<0.59$. In Table 2 , we compare this calibration with the spectroscopic catalogue, in the same way as for the empirically derived metallicity calibrations. We find an offset of 0.33 dex with a scatter of 0.3 dex. This calibration clearly reproduces the spectroscopically derived iron abundances more poorly than the empirical calibrations available in the literature. This shortcoming of the theoretical calibrations was already noted and discussed by Önehag et al. (2009).

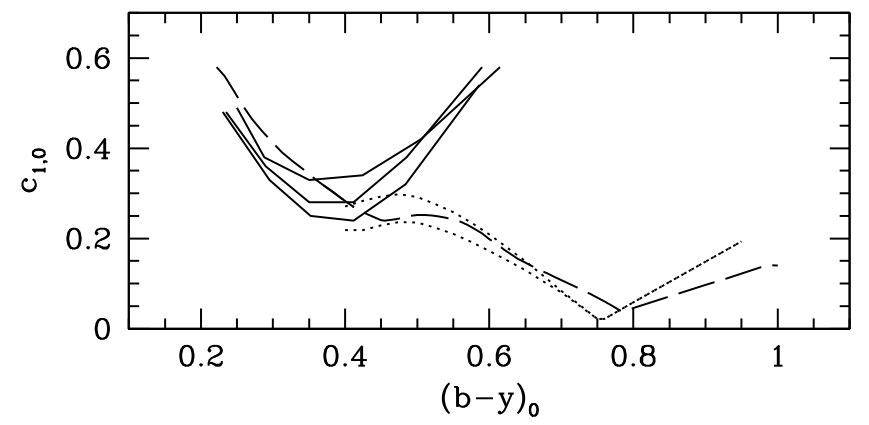

Fig. 17. A comparison of dwarf star sequences, as derived in this paper, for $[\mathrm{Fe} / \mathrm{H}]$ of 0 , and -0.5 dex (dotted lines) with stellar indices, for stars with $\log g=4.5$ and $[\mathrm{Fe} / \mathrm{H}]$ of $0,-0.5$, and $-1.0 \mathrm{dex}$, from Önehag et al. (2009) (solid lines). The preliminary sequences by Olsen (1984) and by Crawford (1975) are also shown (long dashed line).
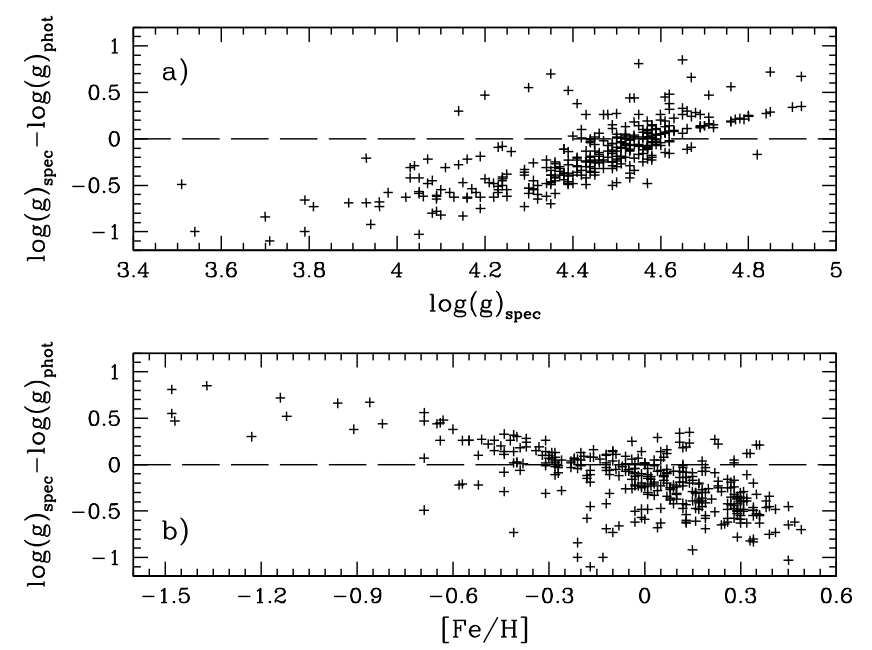

Fig. 18. A comparison of $\log g$ determined using the photometric calibration by Olsen (1984) and $\log g$ determined from an abundance analysis based on high resolution spectroscopy (Valenti \& Fischer 2005). a) The difference as a function of $\log g$ determined in the spectroscopic analysis. b) The difference as a function of $[\mathrm{Fe} / \mathrm{H}]$.

\section{3. log g from uvby photometry - a critical evaluation}

Although the Strömgren system is clearly capable of distinguishing between dwarf and giant stars for colours redder than $(b-y)_{0} \sim 0.55$, the situation is far less clear when we consider the turn-off and sub-giant region. To separate, e.g., field dwarf stars from field sub-giants, we need a measure of their surface gravity for which any metallicity dependence has been taken into account, before being able to distinguish between the dwarf, sub-giant, and giant stars in this narrow colour space (compare Fig. 2).

Hence, it would be desirable to derive $\log g$ directly from the photometry itself. To our knowledge, the only $\log g$ calibration based only on uvby photometry is that of Olsen (1984). If $\beta$ were to be included, additional calibrations would be available (including van Leeuwen 2009; Edvardsson et al. 1993, where the calibration is only shown graphically).

Using the stars in Table B.1 with $\log g$ determinations from Valenti \& Fischer (2005), we test the calibration of Olsen (1984). Figure 18 shows the $\log g$ derived in the spectroscopic study of Valenti \& Fischer (2005) $\left(\log g_{\text {spec }}\right)$ minus the $\log g$ derived from the photometry $\left(\log g_{\text {phot }}\right)$. As can be seen, the calibration has a strong dependence on $[\mathrm{Fe} / \mathrm{H}]$. 

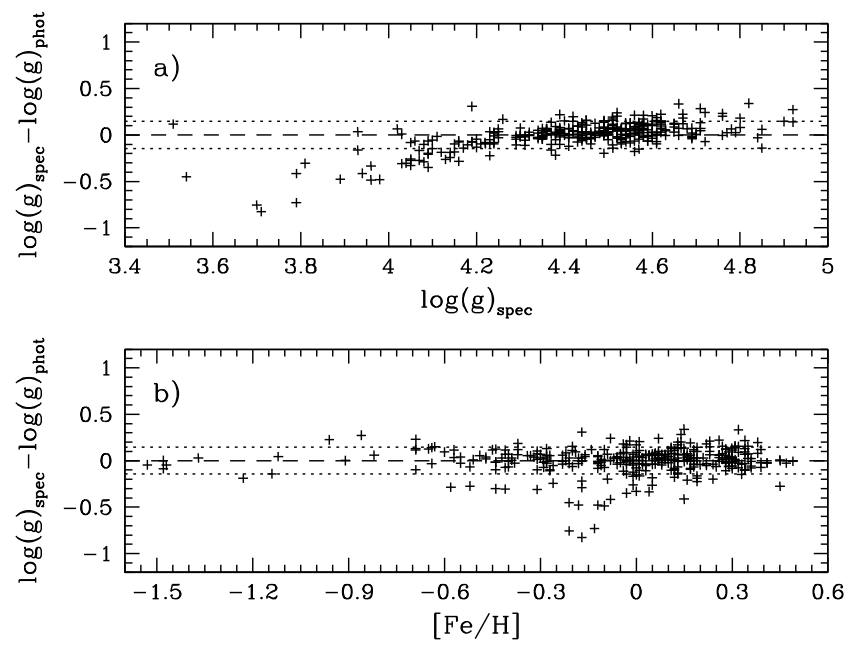

Fig. 19. We show our best attempt at deriving a new $\log g$ calibration from uvby photometry. a) A comparison of $\log g$ determined using Eq. (3) and $\log g$ determined from abundance analysis based on high resolution spectroscopy (Valenti \& Fischer 2005), plotted as a function of spectroscopically determined $\log g$. The mean difference (dashed line) is 0.00 with a $\sigma=0.15$ (dotted line). b) A comparison of $\log g$ determined using Eq. (3) and $\log g$ determined from high resolution spectroscopy (Valenti \& Fischer 2005) as a function of $[\mathrm{Fe} / \mathrm{H}]$. The mean difference (dashed line) is 0.00 with a $\sigma=0.15$ (dotted line).

We now attempt the construction of a new calibration to derive $\log g$ directly from dereddened $u v b y$ photometry, using the spectroscopic catalogue in Table B.1. We start with a third order polynomial in $(b-y)_{0}, m_{1,0}$, and $c_{1,0}$. We note that some calibrations include terms in $[\mathrm{Fe} / \mathrm{H}]$, which we do not because we derive $[\mathrm{M} / \mathrm{H}]$ from the same photometry and hence adding $[\mathrm{M} / \mathrm{H}]$ terms would only mean adding yet more terms to the equation without gaining any further knowledge.

After removing terms that do not contribute significantly, we obtain the fifteenth order polynomial

$$
\begin{aligned}
\log g= & -178.0420(b-y)_{0}+109.7056 m_{1,0} \\
& +47.4263 c_{1,0}+615.0911(b-y)_{0}^{2} \\
& +47.0152 m_{1,0}^{2}-114.8399 c_{1,0}^{2} \\
& -525.0138(b-y)_{0} m_{1,0}-112.5602 m_{1,0} c_{1,0} \\
& -598.8569(b-y)_{0}^{3}+674.8341(b-y)_{0}^{2} m_{1,0} \\
& -267.7717(b-y)_{0}^{2} c_{1,0}-147.5764 m_{1,0}^{2}(b-y)_{0} \\
& +265.3608 c_{1,0}^{2}(b-y)_{0}+266.5860(b-y)_{0} m_{1,0} c_{1,0} \\
& +14.3503 .
\end{aligned}
$$

If we were to include $[\mathrm{Fe} / \mathrm{H}]$ terms the result was a ninth order polynomial. However, as we want to derive both metallicity and surface gravity from the photometry itself, the 15 th order polynomial presented above is a better choice.

Figure 19 shows a comparison with $\log g$ from Table B.1. The comparison is good for stars with $\log g \gtrsim 4.0$ but is progressively poorer towards more evolved star, including the regime where we would most need a good calibration to separate dwarf and sub-giant stars with similar colours! Hence, the use of our new calibration is limited to $\log g>4.0$. Equation (3) is calibrated using dwarf stars in the parameter ranges $0.236<(b-$ $y)_{0}<0.616,0.122<c_{1,0}<0.441,0.075<m_{1,0}<0.679$, and $-1.64<[\mathrm{Fe} / \mathrm{H}]<0.49$.
We also considered restricting ourselves to the region of the $c_{1,0}-(b-y)_{0}$ plane where we most need a calibration $\left((b-y)_{0}<\right.$ $0.55,0.24<c_{1,0}<0.44$, and $\left.c_{1,0}<-1.504 *(b-y)_{0}+1.147\right)$. This also failed in the same way as described for the wider parameter ranges, i.e. we were not able to reliably determine the $\log g$ s for subgiant stars. We also attempted to make a calibration that would retrieve the original $\log g \mathrm{~s}$ in a synthetic stellar population, this also failed. Hence, there does not appear to be an easy, straightforward way to derive $\log g$ directly from the Strömgren $u v b y$ photometry for turn-off and subgiant stars.

Based on their theoretical investigation, Önehag et al. (2009) find that for dwarf stars cooler than the Sun $c_{1,0}$ is not a good measure of stellar gravity. However, from our empirical comparison of $\log g$ derived using the calibration by Olsen (1984) and from spectroscopy we find that for stars redder than $(b-y)_{0} \sim 0.5$ the spectroscopic $\log g$ compares very well with the photometric $\log g$. For stars with $\log g \sim 4.5$, the comparison is also good. It thus seems that for main sequence, cool dwarf stars the Strömgren system is able to predict the surface gravity of the star.

\section{Summary}

As part of our studies of the properties of the Milky Way disk system we have undertaken a critical evaluation of the Strömgren system's ability to provide accurate stellar parameters and to distinguish between dwarf, sub-giant, and giant stars.

We have found that the metallicity calibration for dwarf stars by Ramírez \& Meléndez (2005a) is the most widely applicable calibration for determining metallicities for dwarf and subgiant stars. The calibration of Olsen (1984) provides an extension from $(b-y)_{0}=0.8$ to $(b-y)_{0}=1.0$. We also note that the older calibration of Schuster \& Nissen (1989b) performs almost equally well, but it does not extend to as red colours as the calibration of Ramírez \& Meléndez (2005a).

Although we have found that $u v b y$ photometry can readily distinguish between giant and dwarf stars for redder colours, it is disconcerting that no calibration of $\log g$, for dwarf and subgiant stars, is able to reproduce $\log g$ derived from either spectra or Hipparcos parallaxes. In his provisional calibration van Leeuwen (2009) also notes the same.

Using the catalogues of Olsen (1993), Olsen (1994a), and Olsen (1994b) and the metallicity calibration of Ramírez \& Meléndez (2005a), we have traced new, improved standard sequences for dwarf stars. These new sequences are metallicity dependent and provide crucial calibrations for, e.g., stellar isochrones.

Even though we have found that stellar isochrones in the uvby system reasonably well reproduce empirical stellar sequences it is clear that the disagreement between theoretically calculated Strömgren indices and observed ones can be large. This appears somewhat surprising as stellar isochrones employ the same type of model atmospheres to get the predicted colours as is often used for the elemental abundance studies. This state of affairs is unsatisfactory and we encourage future theoretical studies to resolve these problems.

As part of this work, we have compiled a catalogue of dwarf stars with uvby photometry as well as $[\mathrm{Fe} / \mathrm{H}]$ derived from high-resolution, high $\mathrm{S} / \mathrm{N}$ spectroscopy. The iron abundances have been homogenised to the scale provided by Valenti $\&$ Fischer (2005). This catalogue is provided in full (in electronic form) with this paper.

Acknowledgements. We would like to thank the anonymous referee for pointing out the work done by Ramírez \& Meléndez (2004) on the metallicity calibration 
of Strömgren photometry for giant stars. Bengt Gustafsson is thanked for a careful reading of the penultimate manuscript and the provision of numerous detailed comments and discussions that improved both the content and the style of the paper. S.F. is a Royal Swedish Academy of Sciences Research Fellow supported by a grant from the Knut and Alice Wallenberg Foundation. This research has made use of the SIMBAD database, operated at CDS, Strasbourg, France.

\section{References}

Abazajian, K. N., Adelman-McCarthy, J. K., Agüeros, M. A., et al. 2009, ApJS, 182,543

Adén, D., Feltzing, S., Koch, A., et al. 2009a, A\&A, 506, 1147

Adén, D., Wilkinson, M. I., Read, J. I., et al. 2009b, ApJ, 706, L150

Alonso, A., Arribas, S., \& Martinez-Roger, C. 1996, A\&AS, 117, 227

Alonso, A., Arribas, S., \& Martínez-Roger, C. 1999, A\&AS, 140, 261

Anthony-Twarog, B. J., \& Twarog, B. A. 1994, AJ, 107, 1577

Anthony-Twarog, B. J., \& Twarog, B. A. 1998, AJ, 116, 1922

Arce, H. G., \& Goodman, A. A. 1999, ApJ, 512, L135

Arellano Ferro, A., \& Mendoza V., E. E. 1993, AJ, 106, 2516

Beers, T. C., Drilling, J. S., Rossi, S., et al. 2002, AJ, 124, 931

Bell, R. A., \& Gustafsson, B. 1978, A\&AS, 34, 229

Bensby, T., Feltzing, S., Lundström, I., \& Ilyin, I. 2005, A\&A, 433, 185

Bond, H. E. 1970, ApJS, 22, 117

Bond, H. E. 1980, ApJS, 44, 517

Bonfils, X., Delfosse, X., Udry, S., et al. 2005, A\&A, 442, 635

Bonifacio, P., Caffau, E., \& Molaro, P. 2000, A\&AS, 145, 473

Calamida, A., Bono, G., Stetson, P. B., et al. 2007, ApJ, 670, 400

Calamida, A., Bono, G., Stetson, P. B., et al. 2009, ApJ, 706, 1277

Carney, B. W. 1983, AJ, 88, 623

Carollo, D., Beers, T. C., Lee, Y. S., et al. 2008, Nature, 451, 216

Chen, Y. Q., Nissen, P. E., Zhao, G., Zhang, H. W., \& Benoni, T. 2000, A\&AS, 141,491

Clausen, J. V., Larsen, S. S., Garcia, J. M., Gimenez, A., \& Storm, J. 1997, A\&AS, 122, 559

Clem, J. L., VandenBerg, D. A., Grundahl, F., \& Bell, R. A. 2004, AJ, 127, 1227

Cohen, J. G., \& Huang, W. 2009, ApJ, 701, 1053

Cousins, A. W. J. 1987, South African Astron. Obs. Circ., 11, 93

Crawford, D. L. 1975, AJ, 80, 955

Crawford, D. L., \& Barnes, J. V. 1970, AJ, 75, 978

Edvardsson, B., Andersen, J., Gustafsson, B., et al. 1993, A\&A, 275, 101

ESA 1997, VizieR Online Data Catalog, 1239, 0

Faria, D. 2006, Photometry of resolved stellar populations in local group galaxies (Ph.D. Thesis), Lund Observatory

Faria, D., Feltzing, S., Lundström, I., et al. 2007, A\&A, 465, 357

Favata, F., Micela, G., \& Sciortino, S. 1997, A\&A, 323, 809

Feltzing, S., \& Gustafsson, B. 1998, A\&AS, 129, 237

Ferguson, A. M. N., Johnson, R. A., Faria, D. C., et al. 2005, ApJ, 622, L109

Flynn, C., \& Morell, O. 1997, MNRAS, 286, 617

Gilmore, G., \& Reid, N. 1983, MNRAS, 202, 1025

Golay, M. 1974, Introduction to astronomical photometry, Astrophys. Space Sci. Libr., 41

Grebel, E. K., \& Richtler, T. 1992, A\&A, 253, 359

Grønbech, B., Olsen, E. H., \& Strömgren, B. 1976, A\&AS, 26, 155

Grundahl, F., Stetson, P. B., \& Andersen, M. I. 2002, A\&A, 395, 481

Gustafsson, B., \& Ardeberg, A. 1978, in Astronomical Papers Dedicated to Bengt Stromgren, ed. A. Reiz, \& T. Andersen, 145

Gustafsson, B., \& Bell, R. A. 1979, A\&A, 74, 313

Gustafsson, B., \& Nissen, P. E. 1972, A\&A, 19, 261

Haywood, M. 2002, MNRAS, 337, 151

Heiter, U., \& Luck, R. E. 2003, AJ, 126, 2015

Helmi, A., Ivezić, Ž., Prada, F., et al. 2003, ApJ, 586, 195

Hilker, M. 2000, A\&A, 355, 994

Holmberg, J., Nordström, B., \& Andersen, J. 2007, A\&A, 475, 519

Ibata, R., Irwin, M., Lewis, G., Ferguson, A. M. N., \& Tanvir, N. 2001, Nature, 412,49

Ivezić, Ž., Sesar, B., Jurić, M., et al. 2008, ApJ, 684, 287
Johnson, H. L., \& Morgan, W. W. 1953, ApJ, 117, 313

Jønch-Sørensen, H. 1995, A\&A, 298, 799

Kirby, E. N., Simon, J. D., Geha, M., Guhathakurta, P., \& Frebel, A. 2008, ApJ, 685, L43

Kotoneva, E., Flynn, C., Chiappini, C., \& Matteucci, F. 2002, MNRAS, 336, 879 Lagerholm, C. 2008, An investigation of metallicity and memberships of the Sextans dSph galaxy

Landolt, A. U. 1992, AJ, 104, 340

Lee, Y. S., Beers, T. C., Sivarani, T., et al. 2008, AJ, 136, 2050

Lester, J. B., Gray, R. O., \& Kurucz, R. L. 1986, ApJS, 61, 509

Luck, R. E., \& Heiter, U. 2005, AJ, 129, 1063

Malyuto, V. 1994, A\&AS, 108, 441

Martell, S., \& Laughlin, G. 2002, ApJ, 577, L45

Martell, S. L., \& Smith, G. H. 2004, PASP, 116, 920

Mermilliod, J.-C., Mermilliod, M., \& Hauck, B. 1997, A\&AS, 124, 349

Mishenina, T. V., Soubiran, C., Kovtyukh, V. V., \& Korotin, S. A. 2004, A\&A, 418,551

Nissen, P. E. 1981, A\&A, 97, 145

Nordström, B., Mayor, M., Andersen, J., et al. 2004, A\&A, 418, 989

Ochsenbein, F., Bauer, P., \& Marcout, J. 2000, A\&AS, 143, 23

Olsen, E. H. 1983, A\&AS, 54, 55

Olsen, E. H. 1984, A\&AS, 57, 443

Olsen, E. H. 1993, A\&AS, 102, 89

Olsen, E. H. 1994a, A\&AS, 104, 429

Olsen, E. H. 1994b, A\&AS, 106, 257

Olsen, E. H. 1995, A\&A, 295, 710

Önehag, A., Gustafsson, B., Eriksson, K., \& Edvardsson, B. 2009, A\&A, 498, 527

Perry, C. L., Olsen, E. H., \& Crawford, D. L. 1987, PASP, 99, 1184

Perryman, M. A. C., Lindegren, L., Kovalevsky, J., et al. 1997, A\&A, 323, L49

Ramírez, I., \& Meléndez, J. 2004, A\&A, 417, 301

Ramírez, I., \& Meléndez, J. 2005a, ApJ, 626, 446

Ramírez, I., \& Meléndez, J. 2005b, ApJ, 626, 465

Santos, N. C., Israelian, G., \& Mayor, M. 2001, A\&A, 373, 1019

Santos, N. C., Israelian, G., \& Mayor, M. 2004, A\&A, 415, 1153

Santos, N. C., Israelian, G., Mayor, M., et al. 2005, A\&A, 437, 1127

Schlegel, D. J., Finkbeiner, D. P., \& Davis, M. 1998, ApJ, 500, 525

Schuster, W. J., \& Nissen, P. E. 1988, A\&AS, 73, 225

Schuster, W. J., \& Nissen, P. E. 1989a, A\&A, 222, 69

Schuster, W. J., \& Nissen, P. E. 1989b, A\&A, 221, 65

Schuster, W. J., Nissen, P. E., Parrao, L., Beers, T. C., \& Overgaard, L. P. 1996, A\&AS, 117, 317

Schuster, W. J., Beers, T. C., Michel, R., Nissen, P. E., \& García, G. 2004, A\&A, 422, 527

Schuster, W. J., Moitinho, A., Márquez, A., Parrao, L., \& Covarrubias, E. 2006, A\&A, 445, 939

Sousa, S. G., Santos, N. C., Israelian, G., Mayor, M., \& Monteiro, M. J. P. F. G. 2006, A\&A, 458, 873

Strigari, L. E., Bullock, J. S., Kaplinghat, M., et al. 2008, Nature, 454, 1096

Strömgren, B. 1963, QJRAS, 4, 8

Strömgren, B. 1964, Astrophys. Norvegica, 9, 333

Thorén, P., \& Feltzing, S. 2000, A\&A, 363, 692

Twarog, B. A., Anthony-Twarog, B. J., \& De Lee, N. 2003, AJ, 125, 1383

Twarog, B. A., Vargas, L. C., \& Anthony-Twarog, B. J. 2007, AJ, 134, 1777

Valenti, J. A., \& Fischer, D. A. 2005, ApJS, 159, 141

van Leeuwen, F. 2007, Hipparcos, the New Reduction of the Raw Data, Institute of Astronomy, Cambridge University, Cambridge, UK Series, Astrophys. Space Sci. Libr. (Dordrecht: Springer), 350, 20

van Leeuwen, F. 2009, A\&A, 497, 209

Vandenberg, D. A., \& Bell, R. A. 1985, ApJS, 58, 561

VandenBerg, D. A., Bergbusch, P. A., \& Dowler, P. D. 2006, ApJS, 162, 375

von Hippel, T. A. 1992, AJ, 104, 1765

von Hippel, T., \& Bothun, G. D. 1993, ApJ, 407, 115

Woolf, V. M., \& Wallerstein, G. 2005, MNRAS, 356, 963

Yasuda, N., Fukugita, M., \& Schneider, D. P. 2007, AJ, 134, 698

Yong, D., \& Lambert, D. L. 2003, PASP, 115, 22

York, D. G., Adelman, J., Anderson, Jr., J. E., et al. 2000, AJ, 120, 1579

Pages 19 to 34 are available in the electronic edition of the journal at http: //www . aanda. org 
A. S. Árnadóttir et al.: Metallicities and stellar classification from Strömgren photometry

Appendix A: Stellar sequences

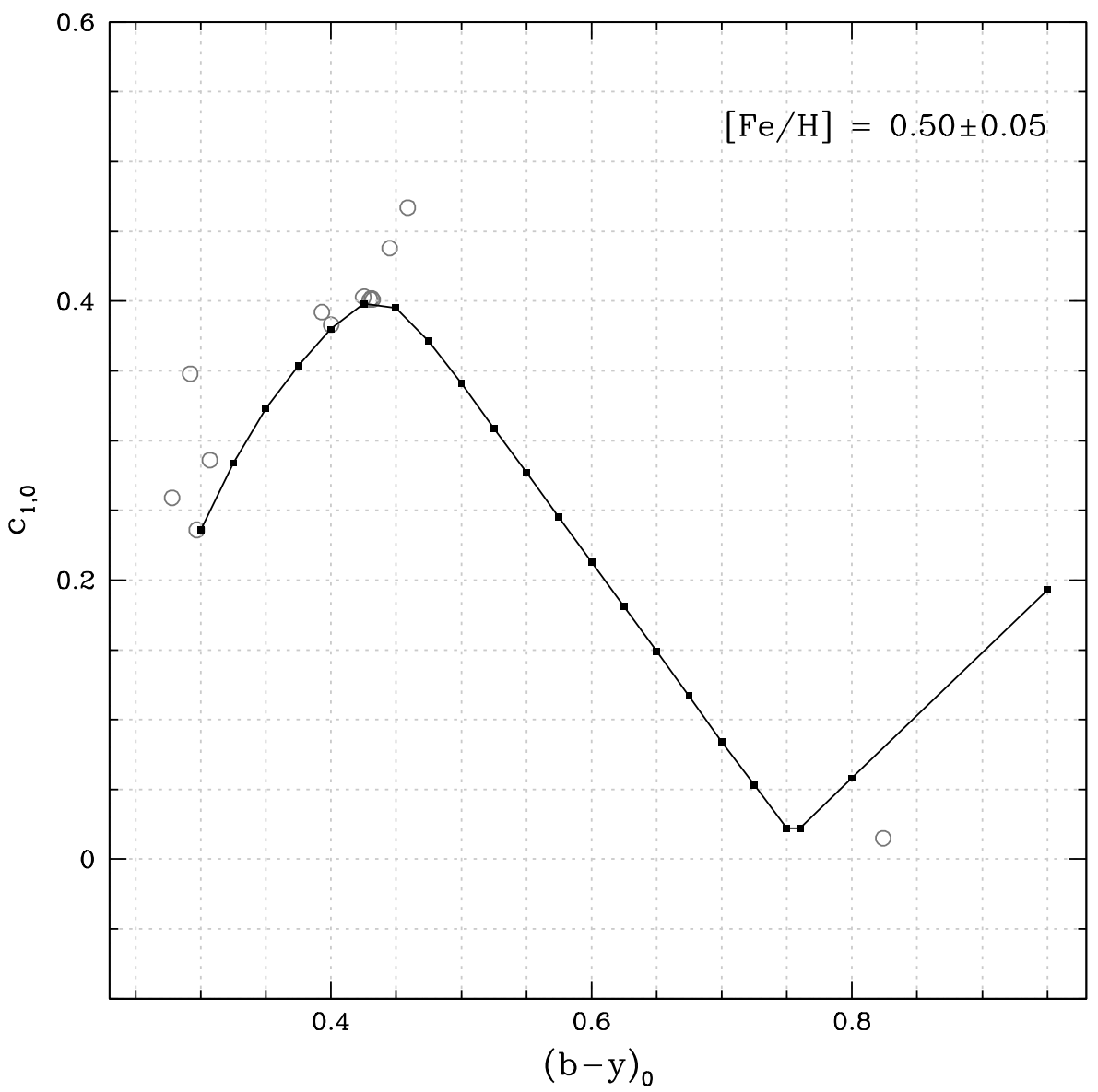

Fig. A.1. The figure shows how the dwarf star sequence was traced from nearby dwarf stars with $[\mathrm{Fe} / \mathrm{H}]=0.50 \pm 0.05$ plotted in the $c_{1,0}$ vs. $(b-y)_{0}$ diagram. 
A\&A 521, A40 (2010)
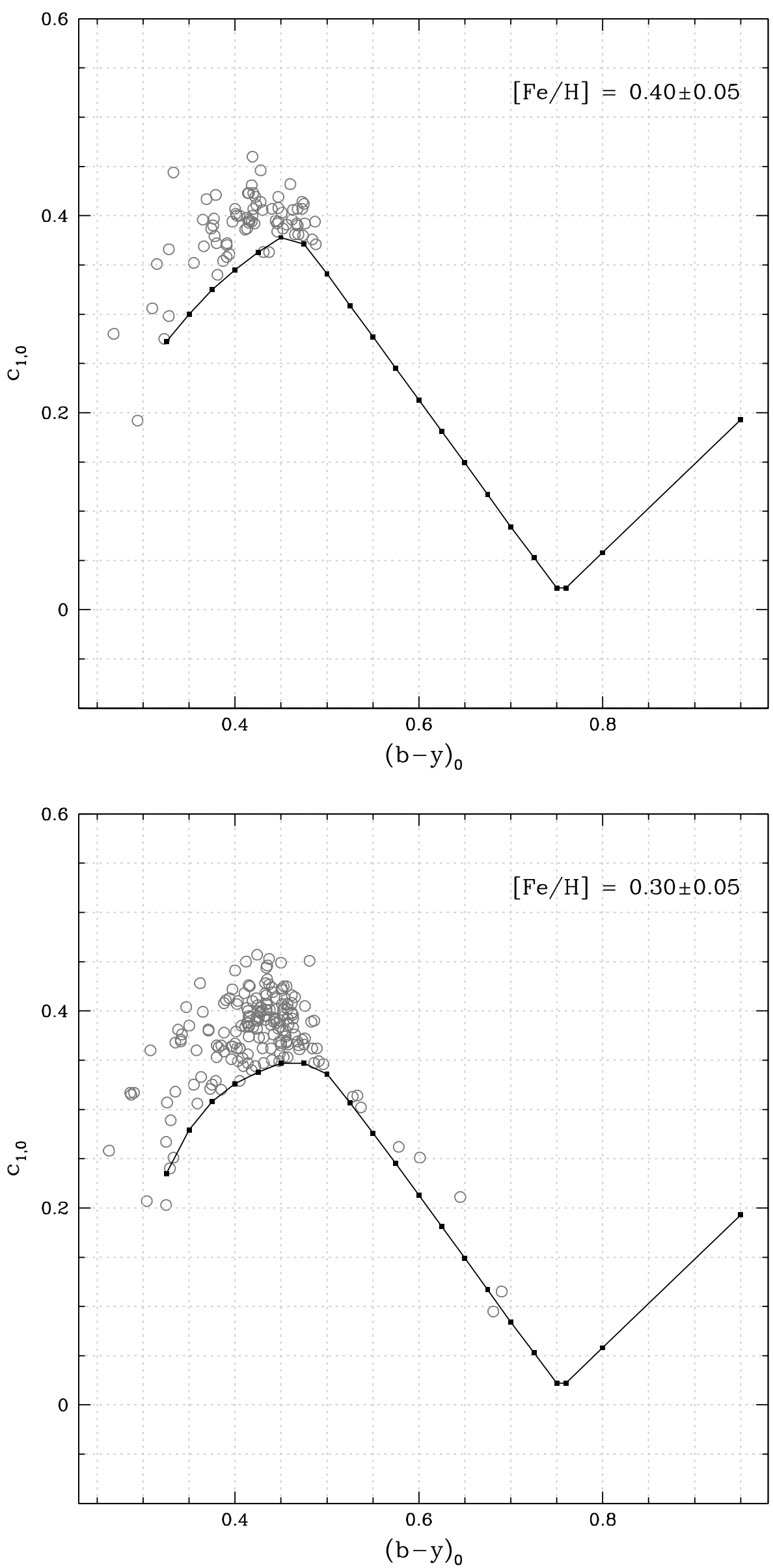

Fig. A.2. The figure shows how the dwarf star sequence was traced from nearby dwarf stars with $[\mathrm{Fe} / \mathrm{H}]=0.40 \pm 0.05$ plotted in the $c_{1,0}$ vs. $(b-y)_{0}$ diagram.
Fig. A.3. The figure shows how the dwarf star sequence was traced from nearby dwarf stars with $[\mathrm{Fe} / \mathrm{H}]=0.30 \pm 0.05$ plotted in the $c_{1,0}$ vs. $(b-y)_{0}$ diagram. 
A. S. Árnadóttir et al.: Metallicities and stellar classification from Strömgren photometry
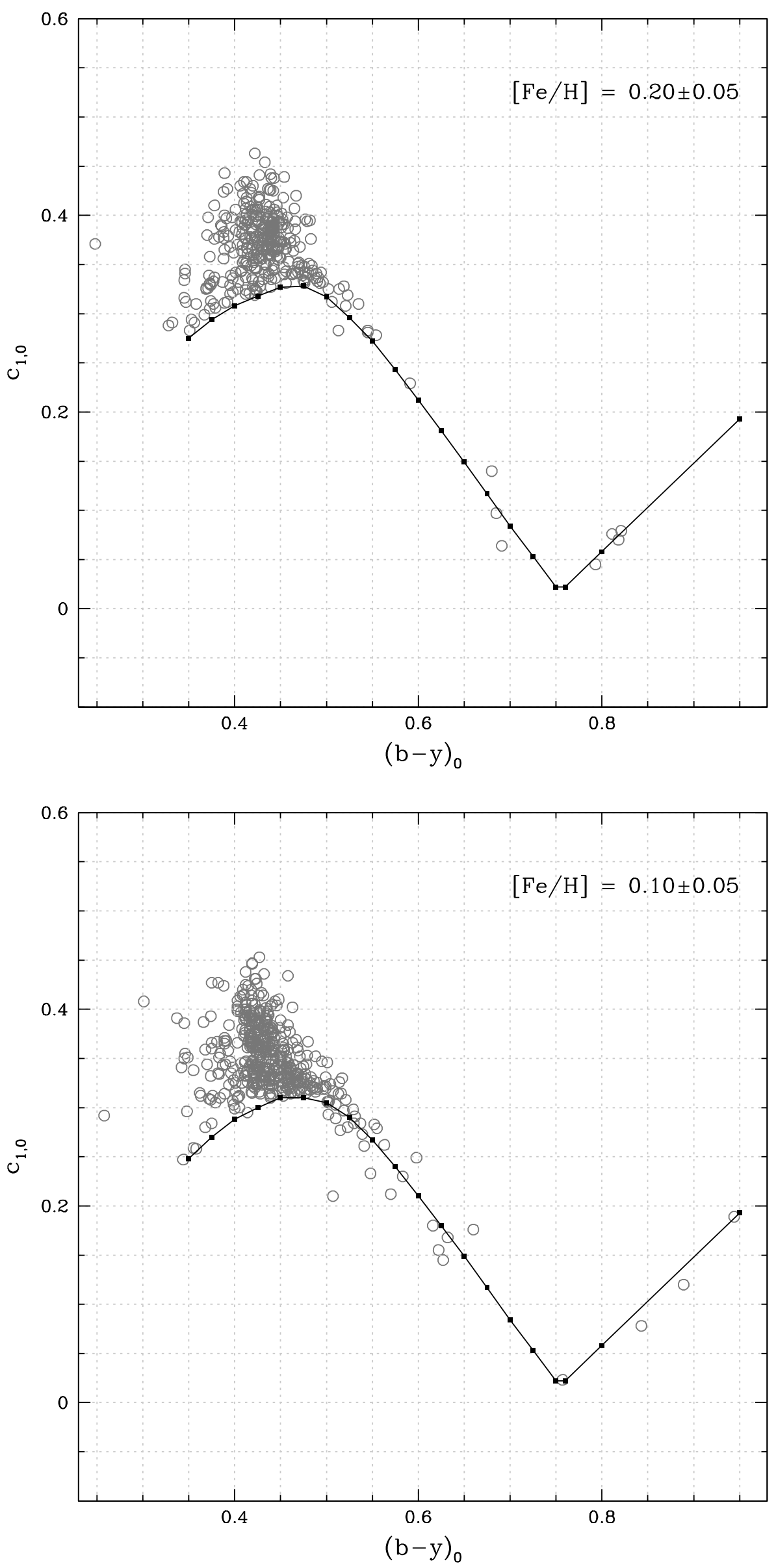

Fig. A.4. The figure shows how the dwarf star sequence was traced from nearby dwarf stars with $[\mathrm{Fe} / \mathrm{H}]=0.20 \pm 0.05$ plotted in the $c_{1,0}$ vs. $(b-y)_{0}$ diagram.

Fig. A.5. The figure shows how the dwarf star sequence was traced from nearby dwarf stars with $[\mathrm{Fe} / \mathrm{H}]=0.10 \pm 0.05$ plotted in the $c_{1,0}$ vs. $(b-y)_{0}$ diagram. 
A\&A 521, A40 (2010)
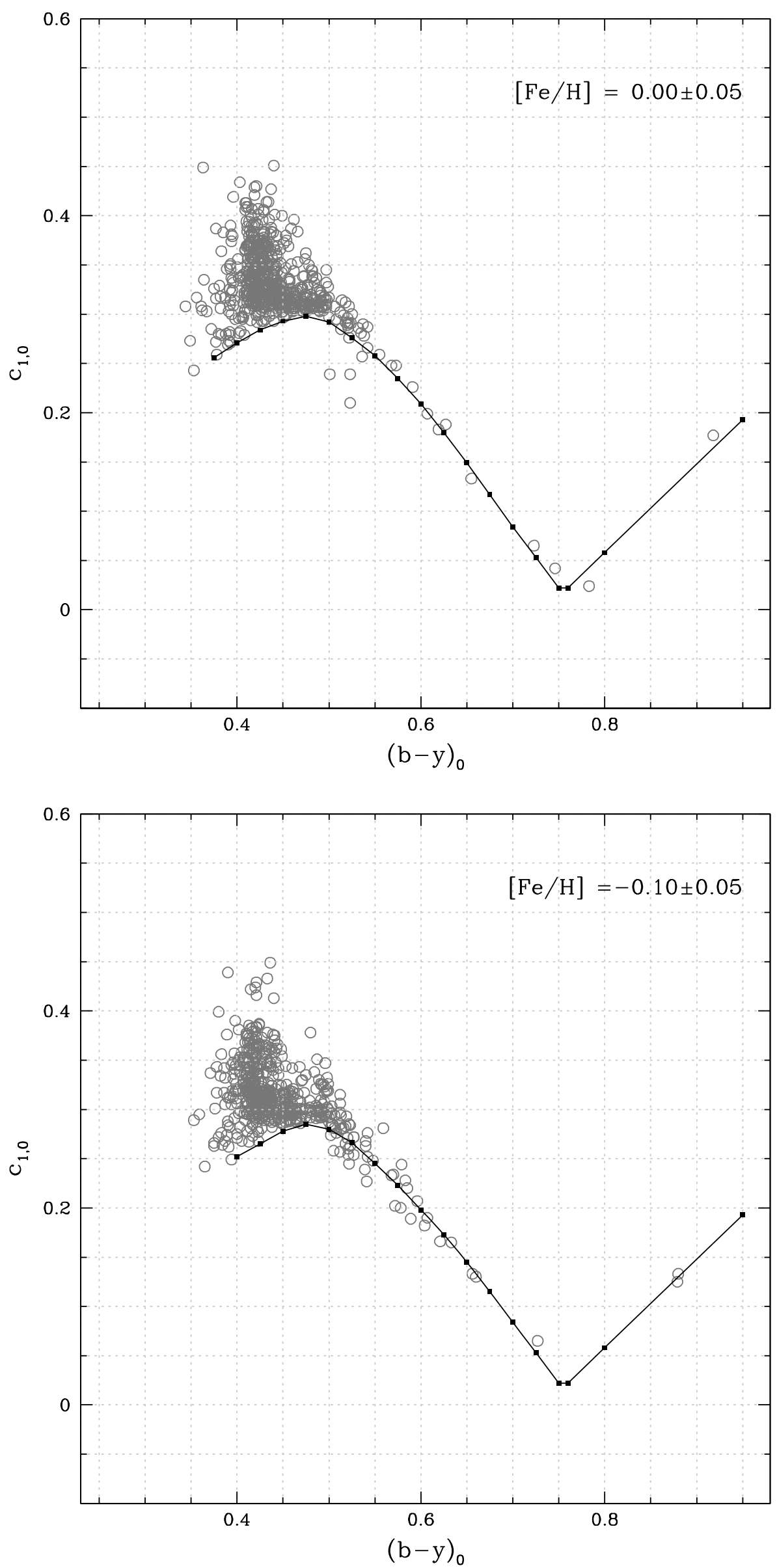

Fig. A.6. The figure shows how the dwarf star sequence was traced from nearby dwarf stars with $[\mathrm{Fe} / \mathrm{H}]=0.00 \pm 0.05$ plotted in the $c_{1,0}$ vs. $(b-y)_{0}$ diagram.

Fig. A.7. The figure shows how the dwarf star sequence was traced from nearby dwarf stars with $[\mathrm{Fe} / \mathrm{H}]=-0.10 \pm 0.05$ plotted in the $c_{1,0}$ vs. $(b-y)_{0}$ diagram. 
A. S. Árnadóttir et al.: Metallicities and stellar classification from Strömgren photometry
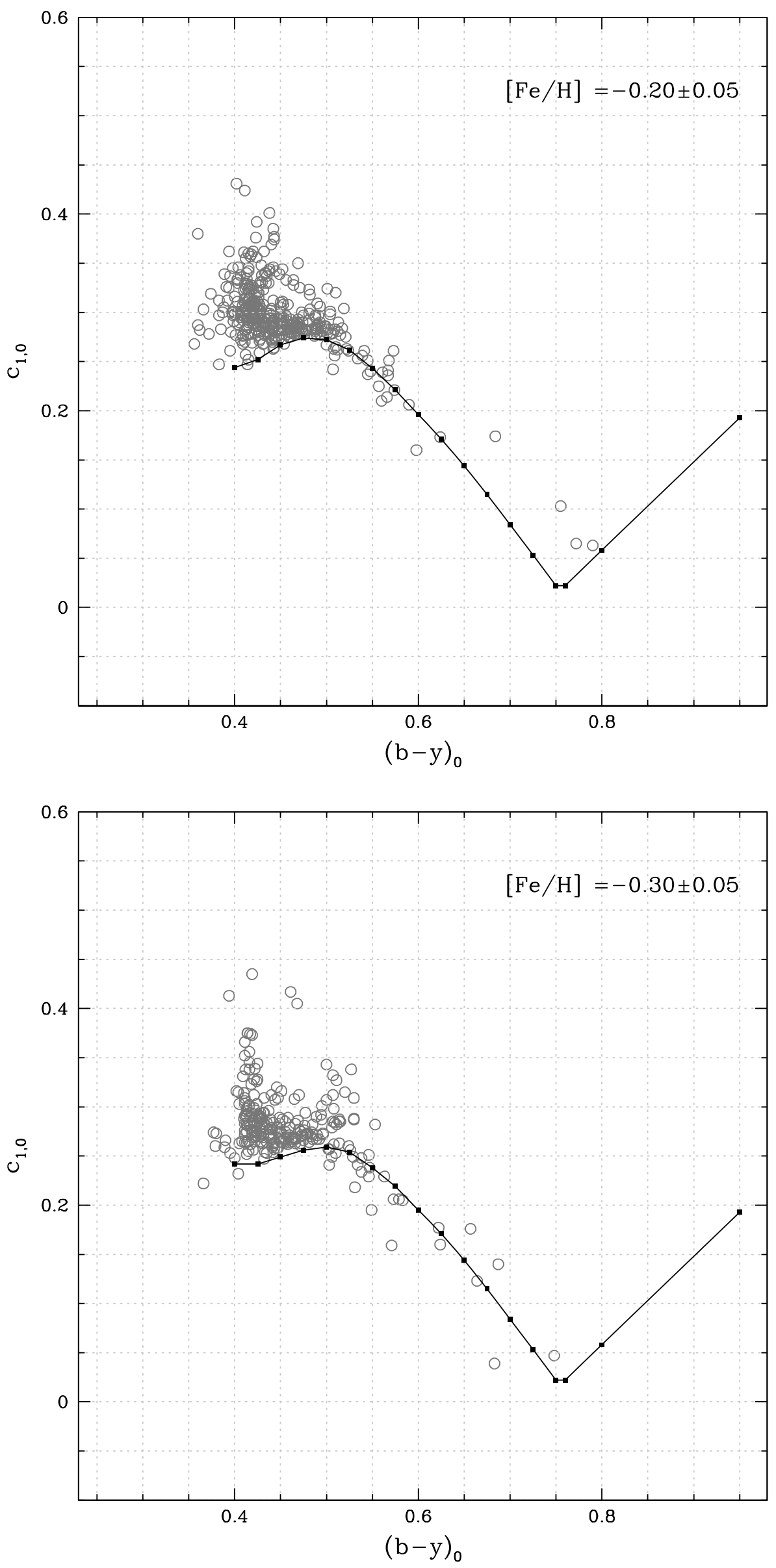

Fig. A.8. The figure shows how the dwarf star sequence was traced from nearby dwarf stars with $[\mathrm{Fe} / \mathrm{H}]=-0.20 \pm 0.05$ plotted in the $c_{1,0}$ vs. $(b-y)_{0}$ diagram.

Fig. A.9. The figure shows how the dwarf star sequence was traced from nearby dwarf stars with $[\mathrm{Fe} / \mathrm{H}]=-0.30 \pm 0.05$ plotted in the $c_{1,0}$ vs. $(b-y)_{0}$ diagram. 
A\&A 521, A40 (2010)
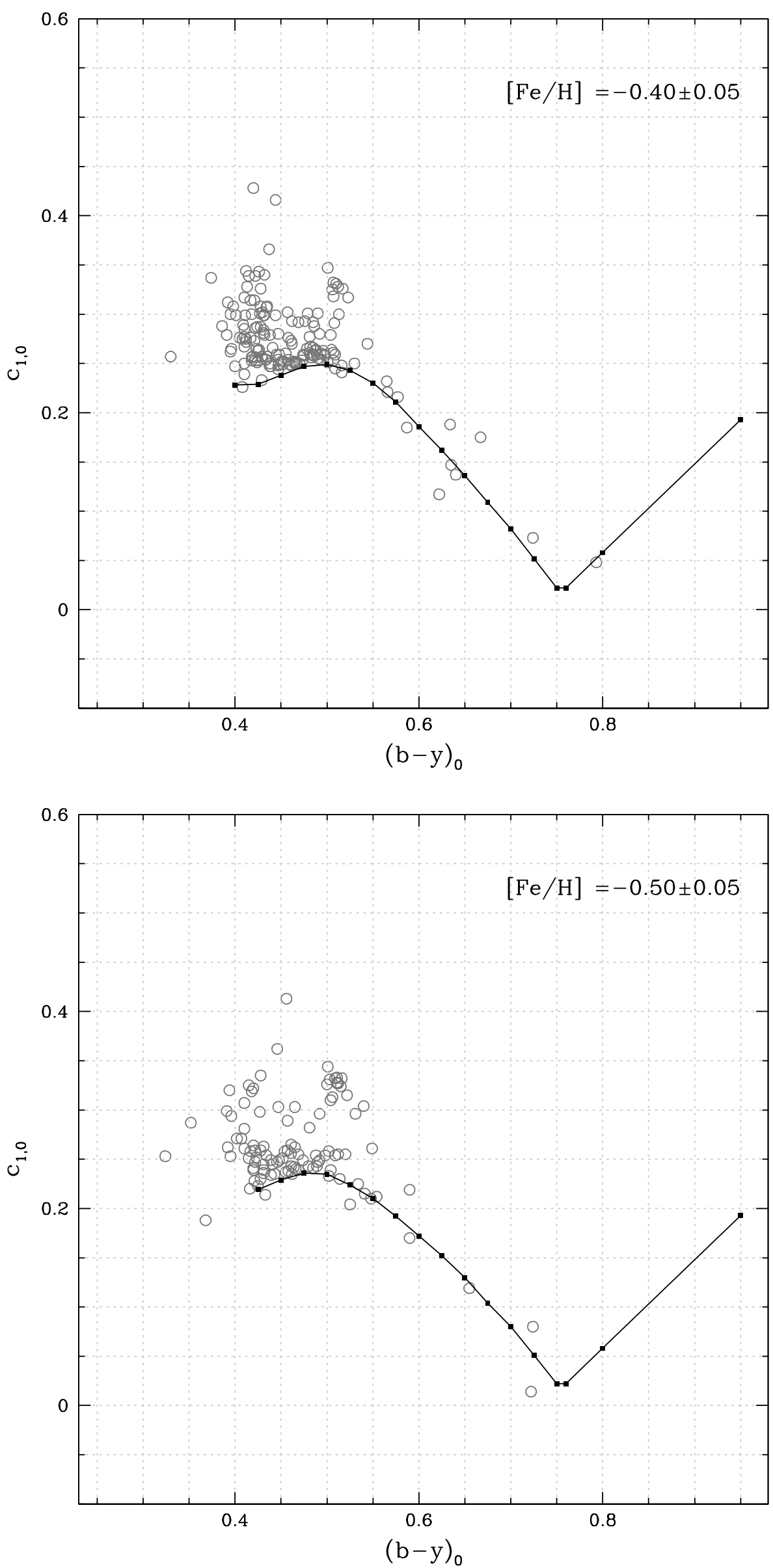

Fig. A.10. The figure shows how the dwarf star sequence was traced from nearby dwarf stars with $[\mathrm{Fe} / \mathrm{H}]=-0.40 \pm 0.05$ plotted in the $c_{1,0}$ vs. $(b-y)_{0}$ diagram.

Fig. A.11. The figure shows how the dwarf star sequence was traced from nearby dwarf stars with $[\mathrm{Fe} / \mathrm{H}]=-0.50 \pm 0.05$ plotted in the $c_{1,0}$ vs. $(b-y)_{0}$ diagram. 
A. S. Árnadóttir et al.: Metallicities and stellar classification from Strömgren photometry
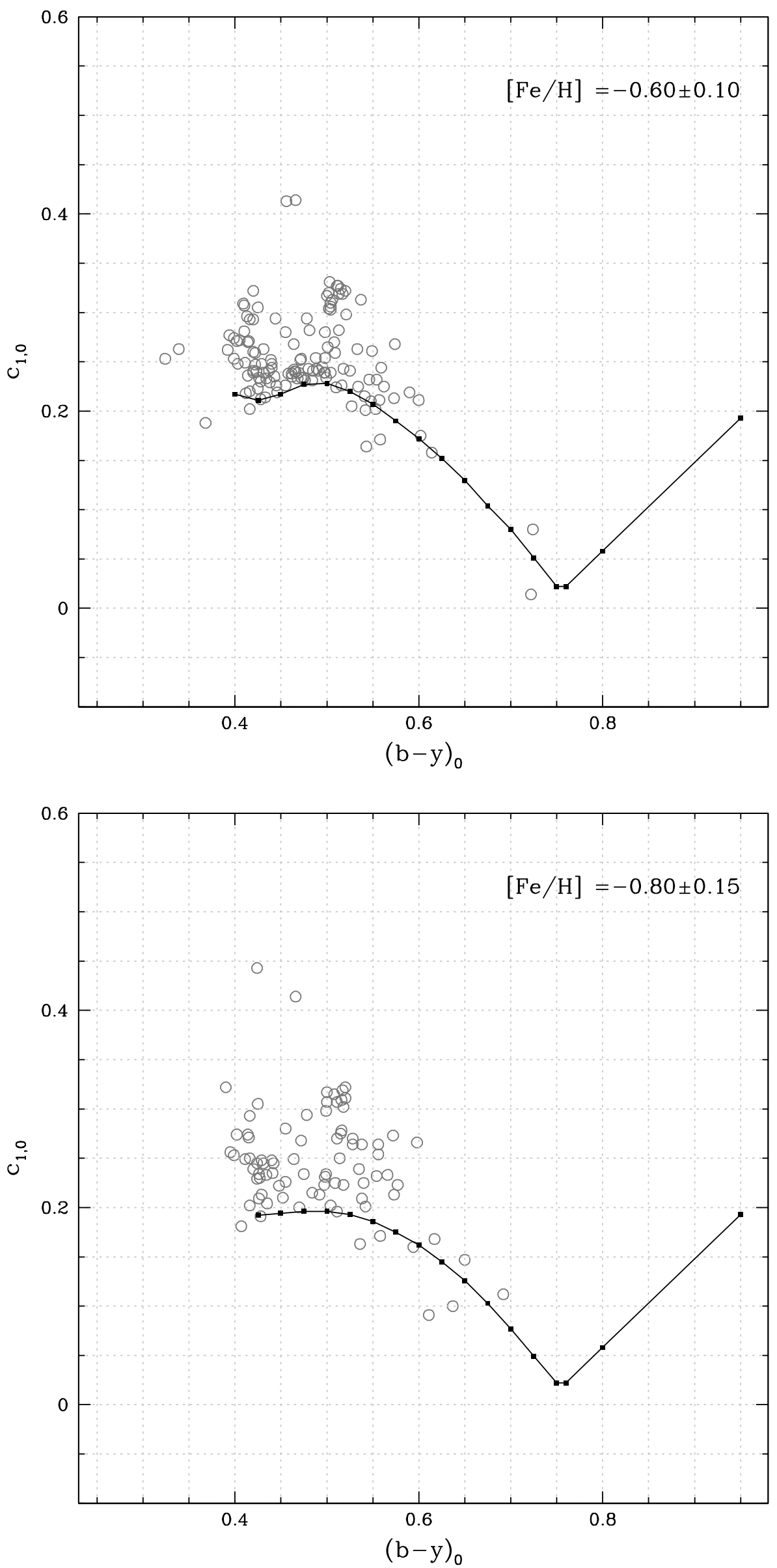

Fig. A.12. The figure shows how the dwarf star sequence was traced from nearby dwarf stars with $[\mathrm{Fe} / \mathrm{H}]=-0.60 \pm 0.10$ plotted in the $c_{1,0}$ vs. $(b-y)_{0}$ diagram.

Fig. A.13. The figure shows how the dwarf star sequence was traced from nearby dwarf stars with $[\mathrm{Fe} / \mathrm{H}]=-0.80 \pm 0.10$ plotted in the $c_{1,0}$ vs. $(b-y)_{0}$ diagram. 
A\&A 521, A40 (2010)

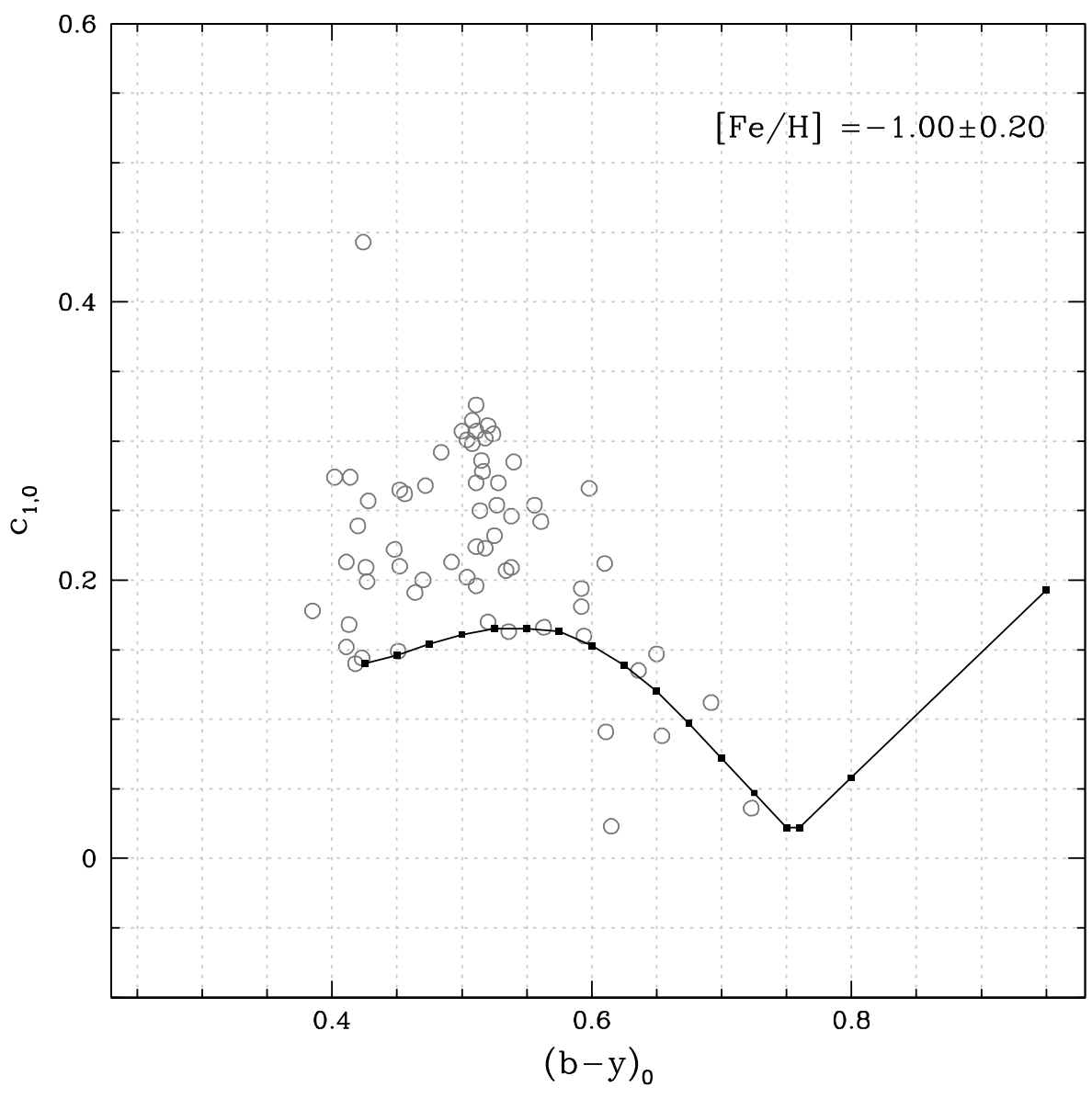

Fig. A.14. The figure shows how the dwarf star sequence was traced from nearby dwarf stars with $[\mathrm{Fe} / \mathrm{H}]=-1.00 \pm 0.20$ plotted in the $c_{1,0}$ vs. $(b-y)_{0}$ diagram. 


\section{Appendix B: Table containing the data collected \\ to test calibrations of $[\mathrm{Fe} / \mathrm{H}]$ in section}

How the catalogue is constructed is explained in detail in Sect. 3.

Column 1 lists the Hipparcos number of the star and Col. 2 gives an alternative stellar name. Column 3 gives the photometry reference (SN88 for Schuster \& Nissen 1988; O84 for Olsen 1984; O93 for Olsen 1993; and O94 for Olsen 1994a) and Columns 4 to 7 give the uvby photometry. Column 8 gives the colour excess of the star. Columns 9 to 12 give the dereddened uvby photometry. Column 13 and 14 give the average $[\mathrm{Fe} / \mathrm{H}]$ (on the Valenti \& Fischer 2005, scale) and the full range of $[\mathrm{Fe} / \mathrm{H}]$ (on the same scale as in Col. 13) if the star was found in more than one study. Columns 15 and 16 give the number of references for the $[\mathrm{Fe} / \mathrm{H}]$ and lists them (1: Valenti \& Fischer 2005; 2: Favata et al. 1997; 3: Feltzing \& Gustafsson 1998; 4: Chen et al. 2000; 5: Thorén \& Feltzing 2000; 6: Santos et al. 2001; 7: Heiter \& Luck 2003; Yong \& Lambert 2003; 9: Mishenina et al. 2004; 10: Santos et al. 2004; 11: Bonfils et al. 2005; 12: Luck \& Heiter 2005; 13: Santos et al. 2005; 14: Woolf \& Wallerstein 2005; and 15: Sousa et al. 2006).

Table B. 1 is also available the CDS. 
A\&A 521, A40 (2010)

Table B.1. Dwarf stars with both Strömgren photometry and $[\mathrm{Fe} / \mathrm{H}]$ based on high resolution, high $\mathrm{S} / \mathrm{N}$ spectroscopy.

\begin{tabular}{|c|c|c|c|c|c|c|c|c|c|c|c|c|c|c|c|}
\hline HIP & Name & $\begin{array}{l}\text { Ref. } \\
\text { uvby }\end{array}$ & V & $(b-y)$ & $m_{1}$ & $c_{1}$ & $E(B-V)$ & $V_{0}$ & $(b-y)_{0}$ & $m_{1,0}$ & $c_{1,0}$ & $\langle[\mathrm{Fe} / \mathrm{H}]\rangle$ & $\begin{array}{r}{[\mathrm{Fe} / \mathrm{H}]} \\
\text { range }\end{array}$ & $\mathrm{N}$ & $\begin{array}{c}\text { Ref. } \\
{[\mathrm{Fe} / \mathrm{H}]}\end{array}$ \\
\hline 400 & HD 225261 & O93 & 7.824 & 0.453 & 0.271 & 0.252 & $<0.02$ & 7.824 & 0.453 & 0.271 & 0.252 & -0.44 & & 1 & 1 \\
\hline 544 & HD 166 & O93 & 6.089 & 0.459 & 0.286 & 0.311 & $<0.02$ & 6.089 & 0.459 & 0.286 & 0.311 & 0.14 & 0.08 & 3 & $1,7,12$ \\
\hline 1499 & HD 1461 & O93 & 6.471 & 0.421 & 0.244 & 0.360 & $<0.02$ & 6.471 & 0.421 & 0.244 & 0.360 & 0.18 & $\ldots$ & 1 & 1 \\
\hline 1931 & HD 2039 & O93 & 9.008 & 0.412 & 0.218 & 0.394 & $<0.02$ & 9.008 & 0.412 & 0.218 & 0.394 & 0.30 & 0.04 & 2 & 1,10 \\
\hline 1936 & HD 2025 & O93 & 7.884 & 0.538 & 0.473 & 0.234 & $<0.02$ & 7.884 & 0.538 & 0.473 & 0.234 & -0.25 & 0.07 & 2 & 1,2 \\
\hline 2282 & HD 2587 & O93 & 8.462 & 0.462 & 0.268 & 0.398 & $<0.02$ & 8.462 & 0.462 & 0.268 & 0.398 & 0.26 & $\ldots$ & 1 & 1 \\
\hline 2790 & HD 3277 & O93 & 7.451 & 0.453 & 0.247 & 0.295 & $<0.02$ & 7.451 & 0.453 & 0.247 & 0.295 & -0.07 & $\ldots$ & 1 & 1 \\
\hline 3170 & HD 3823 & SN88 & 5.907 & 0.363 & 0.146 & 0.353 & $<0.02$ & 5.907 & 0.363 & 0.146 & 0.353 & -0.27 & $\ldots$ & 1 & 1 \\
\hline 3185 & HD 3795 & O93 & 6.148 & 0.443 & 0.217 & 0.282 & $<0.02$ & 6.148 & 0.443 & 0.217 & 0.282 & -0.59 & 0.02 & 2 & 1,2 \\
\hline 3206 & HD 3765 & O93 & 7.353 & 0.537 & 0.494 & 0.302 & $<0.02$ & 7.353 & 0.537 & 0.494 & 0.302 & 0.17 & 0.02 & 2 & 1,9 \\
\hline 3497 & HD 4308 & SN88 & 6.552 & 0.408 & 0.190 & 0.307 & $<0.02$ & 6.552 & 0.408 & 0.190 & 0.307 & -0.32 & 0.02 & 2 & 1,15 \\
\hline 3502 & HD 4203 & O93 & 8.708 & 0.467 & 0.288 & 0.392 & $<0.02$ & 8.708 & 0.467 & 0.288 & 0.392 & 0.43 & 0.04 & 2 & 1,10 \\
\hline 3765 & HD 4628 & O93 & 5.728 & 0.509 & 0.423 & 0.256 & $<0.02$ & 5.728 & 0.509 & 0.423 & 0.256 & -0.25 & 0.04 & 3 & $1,7,12$ \\
\hline 3850 & HD 4747 & O93 & 7.151 & 0.460 & 0.295 & 0.275 & $<0.02$ & 7.151 & 0.460 & 0.295 & 0.275 & -0.22 & 0.01 & 2 & 1,13 \\
\hline 4148 & HD 5133 & O93 & 6.931 & 0.531 & 0.466 & 0.269 & $<0.02$ & 6.931 & 0.531 & 0.466 & 0.269 & -0.09 & 0.08 & 4 & $1,2,6,10$ \\
\hline 5054 & HD 6434 & SN88 & 7.729 & 0.386 & 0.160 & 0.272 & $<0.02$ & 7.729 & 0.386 & 0.160 & 0.272 & -0.57 & 0.08 & 3 & $2,6,10$ \\
\hline 5315 & HD 6734 & O93 & 6.458 & 0.511 & 0.315 & 0.327 & $<0.02$ & 6.458 & 0.511 & 0.315 & 0.327 & -0.41 & $\ldots$ & 1 & 1 \\
\hline 5799 & HD 7438 & O93 & 7.840 & 0.465 & 0.320 & 0.263 & $<0.02$ & 7.840 & 0.465 & 0.320 & 0.263 & -0.26 & $\ldots$ & 1 & 2 \\
\hline 5842 & HD 7693 & O93 & 7.239 & 0.559 & 0.512 & 0.235 & $<0.02$ & 7.239 & 0.559 & 0.512 & 0.235 & 0.23 & $\ldots$ & 1 & 1 \\
\hline 5938 & HD 7661 & O93 & 7.540 & 0.464 & 0.295 & 0.301 & $<0.02$ & 7.540 & 0.464 & 0.295 & 0.301 & 0.06 & $\ldots$ & 1 & 1 \\
\hline 6197 & HD 8038 & $\mathrm{O} 93$ & 8.380 & 0.432 & 0.251 & 0.365 & $<0.02$ & 8.380 & 0.432 & 0.251 & 0.365 & 0.18 & $\ldots$ & 1 & 1 \\
\hline 6442 & HD 8331 & O93 & 7.475 & 0.423 & 0.202 & 0.371 & $<0.02$ & 7.475 & 0.423 & 0.202 & 0.371 & -0.01 & $\ldots$ & 1 & 1 \\
\hline 6498 & HD 8328 & O93 & 8.288 & 0.434 & 0.234 & 0.411 & $<0.02$ & 8.288 & 0.434 & 0.234 & 0.411 & 0.36 & $\ldots$ & 1 & 1 \\
\hline 6653 & HD 8648 & O93 & 7.394 & 0.418 & 0.220 & 0.384 & $<0.02$ & 7.394 & 0.418 & 0.220 & 0.384 & 0.18 & 0.02 & 2 & 1,9 \\
\hline 6712 & HD 8765 & O93 & 8.143 & 0.433 & 0.266 & 0.342 & $<0.02$ & 8.143 & 0.433 & 0.266 & 0.342 & 0.19 & $\ldots$ & 1 & 1 \\
\hline 6978 & HD 9070 & O93 & 7.940 & 0.430 & 0.265 & 0.406 & $<0.02$ & 7.940 & 0.430 & 0.265 & 0.406 & 0.30 & $\ldots$ & 1 & 1 \\
\hline 7080 & HD 9280 & O93 & 8.035 & 0.456 & 0.248 & 0.425 & $<0.02$ & 8.035 & 0.456 & 0.248 & 0.425 & 0.38 & $\ldots$ & 1 & 1 \\
\hline 7221 & HD 9331 & O93 & 8.418 & 0.437 & 0.255 & 0.398 & 0.020 & 8.352 & 0.421 & 0.260 & 0.394 & 0.13 & $\ldots$ & 1 & 1 \\
\hline 7235 & HD 9540 & O93 & 6.971 & 0.451 & 0.291 & 0.294 & $<0.02$ & 6.971 & 0.451 & 0.291 & 0.294 & -0.02 & $\ldots$ & 1 & 1 \\
\hline 7539 & HD 10002 & O94 & 8.130 & 0.502 & 0.370 & 0.344 & $<0.02$ & 8.130 & 0.502 & 0.370 & 0.344 & 0.22 & 0.04 & 2 & 1,15 \\
\hline 7733 & HD 10126 & O93 & 7.736 & 0.445 & 0.267 & 0.312 & $<0.02$ & 7.736 & 0.445 & 0.267 & 0.312 & 0.02 & $\ldots$ & 1 & 1 \\
\hline 7734 & HD 10086 & O93 & 6.617 & 0.419 & 0.237 & 0.339 & $<0.02$ & 6.617 & 0.419 & 0.237 & 0.339 & 0.12 & $\ldots$ & 1 & 1 \\
\hline 7751 & HD 10360 & O94 & 5.739 & 0.500 & 0.392 & 0.267 & $<0.02$ & 5.739 & 0.500 & 0.392 & 0.267 & -0.21 & 0.03 & 3 & $1,6,10$ \\
\hline 7751 & HD 10361 & O94 & 5.863 & 0.512 & 0.421 & 0.262 & $<0.02$ & 5.863 & 0.512 & 0.421 & 0.262 & -0.22 & $\ldots$ & 1 & 1 \\
\hline 7902 & HD 10145 & O93 & 7.713 & 0.421 & 0.232 & 0.326 & 0.035 & 7.597 & 0.394 & 0.242 & 0.320 & 0.01 & 0.05 & 2 & 1,9 \\
\hline 8102 & HD 10700 & O93 & 3.503 & 0.435 & 0.263 & 0.238 & $<0.02$ & 3.503 & 0.435 & 0.263 & 0.238 & -0.53 & 0.05 & 5 & $1,7,12,6,10$ \\
\hline 8159 & HD 10697 & O93 & 6.284 & 0.442 & 0.235 & 0.377 & $<0.02$ & 6.284 & 0.442 & 0.235 & 0.377 & 0.18 & 0.02 & 2 & 1,10 \\
\hline 8346 & HD 11020 & O94 & 8.970 & 0.474 & 0.316 & 0.305 & $<0.02$ & 8.970 & 0.474 & 0.316 & 0.305 & -0.28 & $\ldots$ & 1 & 1 \\
\hline 8362 & HD 10780 & O93 & 5.627 & 0.468 & 0.316 & 0.327 & $<0.02$ & 5.627 & 0.468 & 0.316 & 0.327 & 0.06 & 0.12 & 4 & $1,7,12,9$ \\
\hline 9073 & HD 11850 & O93 & 7.863 & 0.435 & 0.248 & 0.314 & $<0.02$ & 7.863 & 0.435 & 0.248 & 0.314 & 0.09 & $\ldots$ & 1 & 1 \\
\hline 9094 & HD 11964 & SN88 & 6.427 & 0.504 & 0.294 & 0.372 & $<0.02$ & 6.427 & 0.504 & 0.294 & 0.372 & 0.15 & 0.04 & 3 & $1,15,11$ \\
\hline 9269 & HD 12051 & O93 & 7.151 & 0.475 & 0.309 & 0.372 & $<0.02$ & 7.151 & 0.475 & 0.309 & 0.372 & 0.26 & $\ldots$ & 1 & 1 \\
\hline 9381 & HD 12387 & SN88 & 7.376 & 0.410 & 0.196 & 0.316 & $<0.02$ & 7.376 & 0.410 & 0.196 & 0.316 & -0.23 & $\ldots$ & 1 & 1 \\
\hline 10117 & HD 13386 & O94 & 8.894 & 0.514 & 0.418 & 0.325 & $<0.02$ & 8.894 & 0.514 & 0.418 & 0.325 & 0.26 & $\ldots$ & 1 & 15 \\
\hline 10138 & HD 13445 & O94 & 6.113 & 0.483 & 0.337 & 0.286 & $<0.02$ & 6.113 & 0.483 & 0.337 & 0.286 & -0.23 & 0.06 & 3 & $, 6,10$ \\
\hline 10449 & BD -01306 & SN88 & 9.086 & 0.387 & 0.133 & 0.231 & $<0.02$ & 9.086 & 0.387 & 0.133 & 0.231 & -0.96 & $\ldots$ & 1 & 1 \\
\hline 10505 & HD 13825 & O93 & 6.801 & 0.434 & 0.251 & 0.353 & $<0.02$ & 6.801 & 0.434 & 0.251 & 0.353 & 0.19 & $\ldots$ & 1 & 1 \\
\hline 10629 & HD 13783 & O93 & 8.311 & 0.420 & 0.200 & 0.241 & $<0.02$ & 8.311 & 0.420 & 0.200 & 0.241 & -0.73 & $\ldots$ & 1 & 9 \\
\hline 10798 & HD 14412 & O93 & 6.342 & 0.438 & 0.258 & 0.229 & $<0.02$ & 6.342 & 0.438 & 0.258 & 0.229 & -0.49 & 0.06 & 4 & $1,12,6,10$ \\
\hline 11983 & $\mathrm{BD}+4415$ & SN88 & 9.788 & 0.534 & 0.437 & 0.233 & $<0.02$ & 9.788 & 0.534 & 0.437 & 0.233 & -0.46 & . & 1 & 8 \\
\hline 12048 & HD 16141 & SN88 & 6.832 & 0.421 & 0.213 & 0.381 & $<0.02$ & 6.832 & 0.421 & 0.213 & 0.381 & 0.15 & 0.04 & 3 & $1,6,10$ \\
\hline 12114 & HD 16160 & O93 & 5.794 & 0.552 & 0.515 & 0.271 & $<0.02$ & 5.794 & 0.552 & 0.515 & 0.271 & -0.09 & 0.04 & 4 & $1,7,12,11$ \\
\hline 12186 & HD 16417 & O93 & 5.774 & 0.414 & 0.210 & 0.383 & $<0.02$ & 5.774 & 0.414 & 0.210 & 0.383 & 0.14 & 0.03 & 2 & 1,15 \\
\hline 12198 & HD 16275 & O93 & 8.656 & 0.419 & 0.226 & 0.414 & 0.038 & 8.533 & 0.390 & 0.236 & 0.407 & 0.34 & $\ldots$ & 1 & 1 \\
\hline 12306 & HD 16397 & SN88 & 7.361 & 0.387 & 0.157 & 0.279 & $<0.02$ & 7.361 & 0.387 & 0.157 & 0.279 & -0.51 & $\ldots$ & 1 & 1 \\
\hline 13513 & GJ $118.1 \mathrm{~A}$ & O94 & 8.240 & 0.553 & 0.420 & 0.282 & $<0.02$ & 8.240 & 0.553 & 0.420 & 0.282 & 0.17 & $\ldots$ & 1 & 2 \\
\hline 13601 & HD 18144 & O93 & 7.409 & 0.452 & 0.275 & 0.320 & $<0.02$ & 7.409 & 0.452 & 0.275 & 0.320 & 0.07 & $\ldots$ & 1 & 1 \\
\hline 13769 & HD 18445 & O93 & 7.792 & 0.550 & 0.500 & 0.222 & $<0.02$ & 7.792 & 0.550 & 0.500 & 0.222 & 0.00 & $\ldots$ & 1 & 1 \\
\hline 14086 & HD 18907 & $\mathrm{O} 93$ & 5.880 & 0.498 & 0.250 & 0.299 & $<0.02$ & 5.880 & 0.498 & 0.250 & 0.299 & -0.57 & $\ldots$ & 1 & 1 \\
\hline 14241 & HD 19034 & O93 & 8.087 & 0.416 & 0.214 & 0.263 & $<0.02$ & 8.087 & 0.416 & 0.214 & 0.263 & -0.49 & $\ldots$ & 1 & 1 \\
\hline 14286 & HD 18757 & SN88 & 6.652 & 0.408 & 0.202 & 0.313 & $<0.02$ & 6.652 & 0.408 & 0.202 & 0.313 & -0.23 & $\ldots$ & 1 & 11 \\
\hline 14623 & HD 19632 & O93 & 7.292 & 0.421 & 0.230 & 0.329 & $<0.02$ & 7.292 & 0.421 & 0.230 & 0.329 & 0.13 & $\ldots$ & 1 & 1 \\
\hline 15099 & HD 20165 & O93 & 7.812 & 0.503 & 0.404 & 0.296 & 0.036 & 7.695 & 0.476 & 0.414 & 0.290 & -0.03 & $\ldots$ & 1 & 1 \\
\hline 15234 & LHS 170 & SN88 & 10.629 & 0.715 & 0.557 & 0.125 & 0.022 & 10.557 & 0.698 & 0.563 & 0.121 & -1.12 & 0.12 & 2 & 8,14 \\
\hline 15510 & HD 20794 & O93 & 4.256 & 0.439 & 0.235 & 0.285 & $<0.02$ & 4.256 & 0.439 & 0.235 & 0.285 & -0.40 & 0.04 & 3 & $1,6,10$ \\
\hline
\end{tabular}


Table B.1. continued.

\begin{tabular}{|c|c|c|c|c|c|c|c|c|c|c|c|c|c|c|c|}
\hline HIP & Name & $\begin{array}{l}\text { Ref. } \\
\text { uvby }\end{array}$ & V & $(b-y)$ & $m_{1}$ & $c_{1}$ & $E(B-V)$ & $V_{0}$ & $(b-y)_{0}$ & $m_{1,0}$ & $c_{1,0}$ & $\langle[\mathrm{Fe} / \mathrm{H}]\rangle$ & $\begin{array}{r}{[\mathrm{Fe} / \mathrm{H}]} \\
\text { range }\end{array}$ & $\mathrm{N}$ & $\begin{array}{c}\text { Ref. } \\
{[\mathrm{Fe} / \mathrm{H}]}\end{array}$ \\
\hline 15904 & $\mathrm{BD}+11468$ & SN88 & 10.755 & 0.395 & 0.087 & 0.158 & 0.206 & 10.080 & 0.236 & 0.142 & 0.122 & -1.53 & $\ldots$ & 1 & 1 \\
\hline 15919 & HD 21197 & $\mathrm{O} 93$ & 7.841 & 0.645 & 0.729 & 0.149 & $<0.02$ & 7.841 & 0.645 & 0.729 & 0.149 & 0.25 & 0.10 & 3 & $2,15,5$ \\
\hline 16115 & HD 22104 & O93 & 8.311 & 0.423 & 0.229 & 0.403 & $<0.02$ & 8.311 & 0.423 & 0.229 & 0.403 & 0.34 & $\ldots$ & 1 & 1 \\
\hline 16209 & LHS 173 & SN88 & 11.060 & 0.822 & 0.615 & -0.038 & 0.021 & 10.991 & 0.806 & 0.621 & -0.042 & -1.47 & $\ldots$ & 1 & 14 \\
\hline 16405 & HD 21774 & O93 & 8.092 & 0.426 & 0.247 & 0.372 & 0.045 & 7.945 & 0.391 & 0.259 & 0.364 & 0.26 & $\ldots$ & 1 & 1 \\
\hline 16537 & HD 22049 & O93 & 3.716 & 0.498 & 0.436 & 0.257 & $<0.02$ & 3.716 & 0.498 & 0.436 & 0.257 & -0.05 & 0.05 & 5 & $1,12,9,6,10$ \\
\hline 16727 & HD 22282 & O93 & 8.531 & 0.469 & 0.309 & 0.351 & 0.037 & 8.408 & 0.440 & 0.319 & 0.344 & 0.16 & $\ldots$ & 1 & 1 \\
\hline 17054 & HD 23127 & O93 & 8.574 & 0.435 & 0.249 & 0.424 & 0.022 & 8.501 & 0.418 & 0.255 & 0.420 & 0.34 & $\ldots$ & 1 & 1 \\
\hline \multirow[t]{2}{*}{17147} & HD 22879 & SN88 & 6.687 & 0.370 & 0.115 & 0.277 & $<0.02$ & 6.687 & 0.370 & 0.115 & 0.277 & -0.90 & 0.03 & 2 & 1,9 \\
\hline & HD 23261 & O93 & 8.977 & 0.512 & 0.418 & 0.302 & $<0.02$ & 8.977 & 0.512 & 0.418 & 0.302 & 0.04 & $\ldots$ & 1 & 5 \\
\hline 17439 & HD 23484 & O93 & 6.968 & 0.508 & 0.400 & 0.295 & $<0.02$ & 6.968 & 0.508 & 0.400 & 0.295 & 0.11 & 0.02 & 3 & 6,10 \\
\hline 18082 & BD - 4680 & SN88 & 9.977 & 0.388 & 0.049 & 0.288 & 0.136 & 9.530 & 0.283 & 0.086 & 0.264 & -1.57 & $\ldots$ & 1 & 8 \\
\hline 18208 & HD 24365 & O93 & 7.885 & 0.506 & 0.294 & 0.303 & 0.036 & 7.765 & 0.478 & 0.304 & 0.297 & -0.21 & $\ldots$ & 1 & 1 \\
\hline 18309 & HD 24341 & SN88 & 7.881 & 0.438 & 0.184 & 0.291 & $<0.02$ & 7.881 & 0.438 & 0.184 & 0.291 & -0.52 & $\ldots$ & 1 & 1 \\
\hline 18844 & HD 25874 & O93 & 6.735 & 0.416 & 0.217 & 0.336 & $<0.02$ & 6.735 & 0.416 & 0.217 & 0.336 & -0.01 & $\ldots$ & 1 & 1 \\
\hline 19024 & HD 25682 & O93 & 8.480 & 0.457 & 0.293 & 0.335 & 0.051 & 8.312 & 0.418 & 0.307 & 0.326 & 0.09 & $\ldots$ & 1 & 1 \\
\hline 19070 & HD 25790 & O93 & 6.967 & 0.458 & 0.237 & 0.390 & 0.052 & 6.796 & 0.418 & 0.251 & 0.381 & 0.15 & $\ldots$ & 1 & 1 \\
\hline 19232 & HD 26151 & O94 & 8.499 & 0.496 & 0.358 & 0.346 & $<0.02$ & 8.499 & 0.496 & 0.358 & 0.346 & 0.26 & $\ldots$ & 2 & 1,15 \\
\hline 19301 & HD 25918 & O93 & 7.730 & 0.440 & 0.242 & 0.319 & $<0.02$ & 7.730 & 0.440 & 0.242 & 0.319 & -0.05 & $\ldots$ & 1 & 1 \\
\hline 19422 & HD 25665 & O93 & 7.703 & 0.541 & 0.497 & 0.238 & $<0.02$ & 7.703 & 0.541 & 0.497 & 0.238 & -0.03 & $\ldots$ & 1 & 1 \\
\hline 19849 & HD 26965 & O93 & 4.416 & 0.477 & 0.341 & 0.288 & $<0.02$ & 4.416 & 0.477 & 0.341 & 0.288 & -0.26 & 0.06 & 6 & $1,7,12,6,10,11$ \\
\hline 20723 & HD 28185 & O93 & 7.808 & 0.443 & 0.258 & 0.366 & 0.023 & 7.733 & 0.425 & 0.264 & 0.362 & 0.22 & 0.06 & 4 & $7,6,10,15$ \\
\hline 21010 & HD 28447 & O93 & 6.527 & 0.441 & 0.221 & 0.347 & 0.067 & 6.307 & 0.389 & 0.239 & 0.335 & -0.02 & 0.02 & 2 & 1,9 \\
\hline 21436 & HD 29150 & O93 & 7.590 & 0.414 & 0.235 & 0.305 & .033 & 7.482 & 0.389 & 0.244 & 0.299 & 0.02 & 0.06 & 2 & 1,9 \\
\hline 21731 & HD 30306 & O93 & 7.743 & 0.460 & 0.264 & 0.377 & 0.02 & 7.743 & 0.460 & 0.264 & 0.377 & 0.23 & $\ldots$ & 1 & 15 \\
\hline 21850 & HD 30177 & O93 & 8.397 & 0.476 & 0.294 & 0.392 & .02 & 8.397 & 0.476 & 0.294 & 0.392 & 0.40 & 0.01 & 2 & 1,10 \\
\hline 21889 & HD 33 & $\mathrm{O} 93$ & 8.856 & 0.489 & 0.338 & 0.362 & $<0.02$ & 8.856 & 489 & 0.338 & 0.362 & 0.28 & $\ldots$ & 1 & 1 \\
\hline 21923 & HD 29836 & O93 & 7.124 & 0.427 & 0.225 & 0.408 & 0.053 & 6.950 & 0.386 & 0.239 & 0.399 & 0.26 & $\ldots$ & 1 & 1 \\
\hline 21988 & HD 29883 & O93 & 7.985 & 0.526 & 0.431 & 0.282 & 0.024 & 7.908 & 0.508 & 0.437 & 0.278 & -0.18 & $\ldots$ & 1 & 1 \\
\hline 22122 & HD 30501 & $\mathrm{O} 93$ & 7.582 & 0.506 & 0.414 & 0.281 & $<0.02$ & 7.582 & 0.506 & 0.414 & 0.281 & 0.04 & $\ldots$ & 1 & 5 \\
\hline 22319 & HD 30508 & O93 & 6.521 & 0.496 & 0.300 & 0.335 & $<0.02$ & 6.521 & 0.496 & 0.300 & 0.335 & -0.08 & $\ldots$ & 1 & 1 \\
\hline 22336 & HD 30562 & SN88 & 5.770 & 0.395 & 0.216 & 0.409 & $<0.02$ & 5.770 & 0.395 & 0.216 & 0.409 & 0.26 & 0.01 & 4 & $1,3,9,15$ \\
\hline 22451 & HD 30876 & O94 & 7.479 & 0.513 & 0.418 & 0.280 & $<0.02$ & 7.479 & 0.513 & 0.418 & 0.280 & -0.06 & $\ldots$ & 1 & 1 \\
\hline 22576 & HD 30708 & O93 & 6.780 & 0.427 & 0.224 & 0.375 & 0.028 & 6.689 & 0.406 & 0.231 & 0.370 & 0.19 & $\ldots$ & 1 & 1 \\
\hline 22633 & HD 30825 & O93 & 6.726 & 0.517 & 0.311 & 0.320 & 0.072 & 6.489 & 0.461 & 0.330 & 0.307 & -0.17 & $\ldots$ & 1 & 1 \\
\hline 22646 & HD 33214 & O94 & 8.589 & 0.518 & 0.437 & 0.265 & $<0.02$ & 8.589 & 0.518 & 0.437 & 0.265 & 0.17 & $\ldots$ & 1 & 15 \\
\hline 22787 & HD 31392 & O93 & 7.592 & 0.473 & 0.318 & 0.327 & $<0.02$ & 7.592 & 0.473 & 0.318 & 0.327 & 0.05 & $\ldots$ & 1 & 5 \\
\hline 22953 & HD 31827 & O93 & 8.257 & 0.468 & 0.306 & 0.390 & $<0.02$ & 8.257 & 0.468 & 0.306 & 0.390 & 0.41 & $\ldots$ & 1 & 1 \\
\hline 23311 & HD 32147 & O93 & 6.208 & 0.598 & 0.637 & 0.231 & $<0.02$ & 6.208 & 598 & 0.637 & 0.231 & 0.33 & 0.10 & 9 & $2,3,7,12,9$ \\
\hline 23884 & HD 32963 & O93 & 7.606 & 0.410 & 0.228 & 0.335 & 29 & 7.510 & 87 & 0.236 & 0.330 & 0.08 & $\ldots$ & 1 & 1 \\
\hline 24110 & HD 33811 & O93 & 8.711 & 0.465 & 0.283 & 0.409 & $<0.02$ & 8.711 & 0.465 & 0.283 & 0.409 & 0.28 & 0.01 & 2 & 1,2 \\
\hline 25094 & HD 34575 & O93 & 7.095 & 0.458 & 0.264 & 0.404 & $<0.02$ & 7.095 & 0.458 & 0.264 & 0.404 & 0.32 & $\ldots$ & 1 & 1 \\
\hline 25220 & HD 35171 & O93 & 7.929 & 0.624 & 0.650 & 0.160 & $<0.02$ & 7.929 & 0.624 & 0.650 & 0.160 & -0.00 & $\ldots$ & 1 & 12 \\
\hline 25421 & HD 35854 & O94 & 7.694 & 0.536 & 0.501 & 0.257 & $<0.02$ & 7.694 & 0.536 & 0.501 & 0.257 & -0.04 & $\ldots$ & 1 & 1 \\
\hline 25623 & HD 36003 & O93 & 7.623 & 0.627 & 0.655 & 0.188 & $<0.02$ & 7.623 & 0.627 & 0.655 & 0.188 & -0.12 & $\ldots$ & 1 & 12 \\
\hline 25873 & HD 36308 & O93 & 8.363 & 0.465 & 0.312 & 0.303 & 0.112 & 7.995 & 0.378 & 0.342 & 0.283 & 0.15 & $\ldots$ & 1 & 1 \\
\hline 25963 & HD 36889 & O93 & 7.368 & 0.419 & 0.210 & 0.387 & $<0.02$ & 7.368 & 0.419 & 0.210 & 0.387 & 0.16 & $\ldots$ & 1 & 1 \\
\hline 26273 & HD 37213 & O93 & 8.215 & 0.446 & 0.201 & 0.291 & $<0.02$ & 8.215 & 0.446 & 0.201 & 0.291 & -0.44 & $\ldots$ & 1 & 1 \\
\hline 26779 & HD 37394 & O93 & 6.210 & 0.495 & 0.362 & 0.317 & $<0.02$ & 6.210 & 0.495 & 0.362 & 0.317 & 0.13 & 0.10 & 3 & $1,12,11$ \\
\hline 26834 & HD 37986 & $\mathrm{O} 93$ & 7.357 & 0.477 & 0.307 & 0.369 & $<0.02$ & 7.357 & 0.477 & 0.307 & 0.369 & 0.30 & 0.01 & 2 & 3,15 \\
\hline 26935 & HD 38110 & $\mathrm{O} 93$ & 8.180 & 0.416 & 0.209 & 0.399 & 0.153 & 7.678 & 0.298 & 0.250 & 0.372 & 0.17 & $\ldots$ & 1 & 1 \\
\hline 27207 & HD 38230 & O93 & 7.337 & 0.493 & 0.363 & 0.301 & $<0.02$ & 7.337 & 0.493 & 0.363 & 0.301 & -0.08 & $\ldots$ & 1 & 1 \\
\hline 27253 & HD 38529 & O93 & 5.941 & 0.471 & 0.278 & 0.437 & 0.043 & 5.800 & 0.438 & 0.290 & 0.429 & 0.43 & 0.05 & 4 & $1,7,6,10$ \\
\hline 27887 & HD 40307 & O93 & 7.135 & 0.541 & 0.462 & 0.261 & $<0.02$ & 7.135 & 0.541 & 0.462 & 0.261 & -0.25 & 0.06 & 3 & $1,6,10$ \\
\hline 28393 & HD $41004 \mathrm{~A}$ & O94 & 8.639 & 0.518 & 0.392 & 0.312 & $<0.02$ & 8.639 & 0.518 & 0.392 & 0.312 & 0.18 & $\ldots$ & 1 & 13 \\
\hline 29208 & HD 42182 & O93 & 8.434 & 0.520 & 0.460 & 0.295 & $<0.02$ & 8.434 & 0.520 & 0.460 & 0.295 & 0.07 & $\ldots$ & 1 & 5 \\
\hline 29271 & HD 43834 & O94 & 5.073 & 0.442 & 0.269 & 0.329 & $<0.02$ & 5.073 & 0.442 & 0.269 & 0.329 & 0.09 & 0.01 & 3 & $1,6,10$ \\
\hline 29568 & HD 43162 & O93 & 6.364 & 0.428 & 0.246 & 0.303 & $<0.02$ & 6.364 & 0.428 & 0.246 & 0.303 & -0.04 & 0.03 & 2 & 6,10 \\
\hline 29761 & HD 42250 & O93 & 7.433 & 0.469 & 0.287 & 0.333 & $<0.02$ & 7.433 & 0.469 & 0.287 & 0.333 & 0.01 & W & 1 & 1 \\
\hline 29843 & HD 43745 & SN88 & 6.062 & 0.355 & 0.192 & 0.418 & $<0.02$ & 6.062 & 0.355 & 0.192 & 0.418 & 0.12 & 0.02 & 2 & 1,15 \\
\hline 30104 & HD 44594 & O93 & 6.615 & 0.410 & 0.212 & 0.373 & $<0.02$ & 6.615 & 0.410 & 0.212 & 0.373 & 0.15 & $\ldots$ & 1 & 1 \\
\hline 30243 & HD 44420 & O93 & 7.602 & 0.426 & 0.252 & 0.380 & $<0.02$ & 7.602 & 0.426 & 0.252 & 0.380 & 0.29 & $\ldots$ & 1 & 1 \\
\hline 30344 & HD 44821 & O93 & 7.356 & 0.420 & 0.238 & 0.303 & $<0.02$ & 7.356 & 0.420 & 0.238 & 0.303 & 0.11 & $\ldots$ & 1 & 1 \\
\hline 30476 & HD 45289 & $\mathrm{O} 93$ & 6.669 & 0.419 & 0.213 & 0.350 & $<0.02$ & 6.669 & 0.419 & 0.213 & 0.350 & -0.02 & $\ldots$ & 1 & 1 \\
\hline 30860 & HD 45350 & O93 & 7.892 & 0.457 & 0.263 & 0.407 & $<0.02$ & 7.892 & 0.457 & 0.263 & 0.407 & 0.29 & $\ldots$ & 1 & 1 \\
\hline 31246 & HD 46375 & O93 & 7.914 & 0.502 & 0.401 & 0.337 & $<0.02$ & 7.914 & 0.502 & 0.401 & 0.337 & 0.24 & 0.01 & 2 & 1,10 \\
\hline
\end{tabular}


Table B.1. continued.

\begin{tabular}{|c|c|c|c|c|c|c|c|c|c|c|c|c|c|c|c|}
\hline HIP & Name & $\begin{array}{l}\text { Ref. } \\
\text { uvby }\end{array}$ & $V$ & $(b-y)$ & $m_{1}$ & $c_{1}$ & $E(B-V)$ & $V_{0}$ & $(b-y)_{0}$ & $m_{1,0}$ & $c_{1,0}$ & $\langle[\mathrm{Fe} / \mathrm{H}]\rangle$ & $\begin{array}{r}{[\mathrm{Fe} / \mathrm{H}]} \\
\text { range }\end{array}$ & $\mathrm{N}$ & $\begin{array}{c}\text { Ref. } \\
{[\mathrm{Fe} / \mathrm{H}]}\end{array}$ \\
\hline 31540 & HD 47186 & O93 & 7.619 & 0.445 & 0.265 & 0.365 & $<0.02$ & 7.619 & 0.445 & 0.265 & 0.365 & 0.23 & $\ldots$ & 1 & 15 \\
\hline 31655 & HD 47157 & O93 & 7.613 & 0.440 & 0.268 & 0.407 & $<0.02$ & 7.613 & 0.440 & 0.268 & 0.407 & 0.34 & $\ldots$ & 1 & 1 \\
\hline 31660 & HD 47127 & O93 & 6.834 & 0.437 & 0.246 & 0.369 & $<0.02$ & 6.834 & 0.437 & 0.246 & 0.369 & 0.10 & $\ldots$ & 1 & 1 \\
\hline 32010 & HD 47752 & O93 & 8.064 & 0.583 & 0.580 & 0.205 & $<0.02$ & 8.064 & 0.583 & 0.580 & 0.205 & -0.10 & ... & 1 & 1 \\
\hline 32984 & HD 50281 & O93 & 6.572 & 0.592 & 0.607 & 0.206 & $<0.02$ & 6.572 & 0.592 & 0.607 & 0.206 & 0.04 & 0.18 & 8 & $1,2,7,12,9,6,10,11$ \\
\hline 33094 & HD 50806 & O93 & 6.056 & 0.437 & 0.230 & 0.371 & $<0.02$ & 6.056 & 0.437 & 0.230 & 0.371 & 0.07 & $\ldots$ & 1 & 1 \\
\hline 33324 & HD 51929 & SN88 & 7.404 & 0.367 & 0.146 & 0.290 & $<0.02$ & 7.404 & 0.367 & 0.146 & 0.290 & -0.64 & $\ldots$ & 1 & 1 \\
\hline 33382 & HD 51219 & O93 & 7.393 & 0.432 & 0.236 & 0.323 & $<0.02$ & 7.393 & 0.432 & 0.236 & 0.323 & 0.01 & $\ldots$ & 1 & 1 \\
\hline 33690 & HD 53143 & O93 & 6.825 & 0.482 & 0.323 & 0.318 & $<0.02$ & 6.825 & 0.482 & 0.323 & 0.318 & 0.22 & $\ldots$ & 1 & 13 \\
\hline 33848 & HD 52456 & O93 & 8.144 & 0.502 & 0.383 & 0.307 & $<0.02$ & 8.144 & 0.502 & 0.383 & 0.307 & 0.01 & $\ldots$ & 1 & 1 \\
\hline 34065 & HD 53705 & SN88 & 5.557 & 0.383 & 0.194 & 0.319 & $<0.02$ & 5.557 & 0.383 & 0.194 & 0.319 & -0.17 & 0.20 & 4 & $1,2,6,10$ \\
\hline 34069 & HD 53706 & O93 & 6.857 & 0.470 & 0.297 & 0.284 & $<0.02$ & 6.857 & 0.470 & 0.297 & 0.284 & -0.26 & 0.09 & 4 & $1,2,6,10$ \\
\hline 34739 & HD 55720 & O93 & 7.507 & 0.437 & 0.233 & 0.284 & $<0.02$ & 7.507 & 0.437 & 0.233 & 0.284 & -0.30 & $\ldots$ & 1 & 1 \\
\hline 35139 & HD 56274 & SN88 & 7.750 & 0.384 & 0.157 & 0.273 & $<0.02$ & 7.750 & 0.384 & 0.157 & 0.273 & -0.55 & $\ldots$ & 1 & 1 \\
\hline 35910 & HD 58895 & O93 & 6.594 & 0.442 & 0.235 & 0.429 & $<0.02$ & 6.594 & 0.442 & 0.235 & 0.429 & 0.34 & $\ldots$ & 1 & 15 \\
\hline 36210 & HD 59468 & O93 & 6.722 & 0.433 & 0.253 & 0.323 & $<0.02$ & 6.722 & 0.433 & 0.253 & 0.323 & 0.02 & $\ldots$ & 1 & 1 \\
\hline 36249 & HD 58781 & O93 & 7.242 & 0.434 & 0.271 & 0.334 & $<0.02$ & 7.242 & 0.434 & 0.271 & 0.334 & 0.10 & $\ldots$ & 1 & 1 \\
\hline 36285 & HD 58595 & O93 & 7.419 & 0.427 & 0.212 & 0.268 & $<0.02$ & 7.419 & 0.427 & 0.212 & 0.268 & -0.30 & $\ldots$ & 1 & 9 \\
\hline 36704 & HD 59747 & O93 & 7.697 & 0.517 & 0.403 & 0.284 & $<0.02$ & 7.697 & 0.517 & 0.403 & 0.284 & 0.06 & $\ldots$ & 1 & 1 \\
\hline 36849 & HD 60319 & SN88 & 8.929 & 0.351 & 0.112 & 0.302 & $<0.02$ & 8.929 & 0.351 & 0.112 & 0.302 & -0.87 & $\ldots$ & 1 & 4 \\
\hline 37309 & HD 61686 & O94 & 8.549 & 0.419 & 0.230 & 0.387 & $<0.02$ & 8.549 & 0.419 & 0.230 & 0.387 & 0.30 & $\ldots$ & 1 & 1 \\
\hline 37349 & HD 61606 A & O93 & 7.168 & 0.542 & 0.496 & 0.266 & $<0.02$ & 7.168 & 0.542 & 0.496 & 0.266 & 0.03 & 0.06 & 6 & $1,3,12,9,13,5$ \\
\hline 37789 & HD 62301 & SN88 & 6.740 & 0.361 & 0.126 & 0.312 & $<0.02$ & 6.740 & 0.361 & 0.126 & 0.312 & -0.66 & $\ldots$ & 1 & 4 \\
\hline 38558 & HD 65216 & O93 & 7.973 & 0.420 & 0.231 & 0.272 & $<0.02$ & 7.973 & 0.420 & 0.231 & 0.272 & -0.14 & $\ldots$ & 1 & 10 \\
\hline 38625 & HD 64606 & O93 & 7.432 & 0.454 & 0.211 & 0.208 & $<0.02$ & 7.432 & 0.454 & 0.211 & 0.208 & -0.85 & 0.16 & 3 & $2,9,13$ \\
\hline 39064 & HD 65430 & O93 & 7.667 & 0.490 & 0.347 & 0.299 & $<0.02$ & 7.667 & 0.490 & 0.347 & 0.299 & -0.12 & $\ldots$ & 1 & 1 \\
\hline 39157 & HD 65583 & O93 & 6.982 & 0.455 & 0.220 & 0.229 & $<0.02$ & 6.982 & 0.455 & 0.220 & 0.229 & -0.69 & $\ldots$ & 2 & 1,9 \\
\hline 39298 & HD 66221 & O93 & 8.064 & 0.451 & 0.250 & 0.374 & $<0.02$ & 8.064 & 0.451 & 0.250 & 0.374 & 0.17 & . & 1 & 15 \\
\hline 39342 & HD 67199 & O93 & 7.161 & 0.509 & 0.415 & 0.285 & $<0.02$ & 7.161 & 0.509 & 0.415 & 0.285 & 0.06 & 0.06 & 2 & 1,13 \\
\hline 39417 & HD 66428 & O93 & 8.255 & 0.445 & 0.256 & 0.377 & $<0.02$ & 8.255 & 0.445 & 0.256 & 0.377 & 0.31 & $\ldots$ & 1 & 1 \\
\hline 40118 & HD 68017 & O93 & 6.797 & 0.420 & 0.194 & 0.264 & $<0.02$ & 6.797 & 0.420 & 0.194 & 0.264 & -0.42 & 0.03 & 2 & 1,9 \\
\hline 40283 & HD 68978 & SN88 & 6.719 & 0.373 & 0.203 & 0.342 & $<0.02$ & 6.719 & 0.373 & 0.203 & 0.342 & 0.02 & $\ldots$ & 1 & 1 \\
\hline \multirow[t]{2}{*}{40497} & HD 68638 & O93 & 7.509 & 0.457 & 0.276 & 0.267 & $<0.02$ & 7.509 & 0.457 & 0.276 & 0.267 & -0.19 & $\ldots$ & 1 & 9 \\
\hline & HD 69582 & O93 & 7.575 & 0.430 & 0.243 & 0.321 & $<0.02$ & 7.575 & 0.430 & 0.243 & 0.321 & 0.09 & $\ldots$ & 1 & 3 \\
\hline 40693 & HD 69830 & O93 & 5.958 & 0.457 & 0.297 & 0.314 & $<0.02$ & 5.958 & 0.457 & 0.297 & 0.314 & -0.00 & 0.12 & 8 & $1,3,7,12,6,10,15,5$ \\
\hline 40952 & HD 70642 & O93 & 7.169 & 0.435 & 0.252 & 0.350 & $<0.02$ & 7.169 & 0.435 & 0.252 & 0.350 & 0.17 & 0.03 & 3 & $1,10,13$ \\
\hline 41254 & HD 72234 & O93 & 7.173 & 0.421 & 0.186 & 0.362 & 0.022 & 7.100 & 0.404 & 0.192 & 0.358 & -0.12 & $\ldots$ & 1 & 1 \\
\hline 41317 & HD 71334 & O93 & 7.814 & 0.415 & 0.210 & 0.324 & $<0.02$ & 7.814 & 0.415 & 0.210 & 0.324 & -0.06 & $\ldots$ & 1 & 1 \\
\hline 41661 & PLX 2019 & SN88 & 11.919 & 0.549 & 0.387 & 0.160 & $<0.02$ & 11.919 & 0.549 & 0.387 & 0.160 & -1.72 & $\ldots$ & 1 & 8 \\
\hline 41926 & HD 72673 & O93 & 6.379 & 0.473 & 0.291 & 0.258 & $<0.02$ & 6.379 & 0.473 & 0.291 & 0.258 & -0.36 & 0.02 & 4 & $1,2,6,10$ \\
\hline 42011 & HD 72769 & O93 & 7.225 & 0.452 & 0.284 & 0.387 & $<0.02$ & 7.225 & 0.452 & 0.284 & 0.387 & 0.30 & 0.02 & 2 & 1,2 \\
\hline 42074 & HD 72760 & O93 & 7.320 & 0.483 & 0.336 & 0.308 & $<0.02$ & 7.320 & 0.483 & 0.336 & 0.308 & 0.13 & 0.02 & 2 & 1,9 \\
\hline 42214 & HD 73256 & O93 & 8.061 & 0.473 & 0.307 & 0.349 & $<0.02$ & 8.061 & 0.473 & 0.307 & 0.349 & 0.27 & $\ldots$ & 1 & 10 \\
\hline 42282 & HD 73526 & O94 & 8.972 & 0.451 & 0.242 & 0.421 & $<0.02$ & 8.972 & 0.451 & 0.242 & 0.421 & 0.26 & 0.02 & 2 & 1,10 \\
\hline 42499 & HD 73667 & O93 & 7.601 & 0.498 & 0.337 & 0.258 & $<0.02$ & 7.601 & 0.498 & 0.337 & 0.258 & -0.49 & 0.15 & 2 & 1,2 \\
\hline 42808 & HD 74576 & O93 & 6.562 & 0.538 & 0.446 & 0.263 & $<0.02$ & 6.562 & 0.538 & 0.446 & 0.263 & 0.03 & 0.01 & 2 & 6,10 \\
\hline 43686 & HD 76700 & O93 & 8.149 & 0.456 & 0.250 & 0.431 & $<0.02$ & 8.149 & 0.456 & 0.250 & 0.431 & 0.38 & 0.06 & 2 & 1,10 \\
\hline 43852 & HD 76218 & O93 & 7.710 & 0.473 & 0.306 & 0.301 & $<0.02$ & 7.710 & 0.473 & 0.306 & 0.301 & 0.07 & $\ldots$ & 1 & 1 \\
\hline 44075 & HD 76932 & SN88 & 5.801 & 0.354 & 0.117 & 0.297 & $<0.02$ & 5.801 & 0.354 & 0.117 & 0.297 & -1.02 & $\ldots$ & 1 & 9 \\
\hline 44089 & HD 76752 & O93 & 7.484 & 0.412 & 0.208 & 0.351 & $<0.02$ & 7.484 & 0.412 & 0.208 & 0.351 & 0.03 & $\ldots$ & 1 & 1 \\
\hline 44097 & HD 76780 & O93 & 7.648 & 0.414 & 0.234 & 0.361 & $<0.02$ & 7.648 & 0.414 & 0.234 & 0.361 & 0.17 & $\ldots$ & 1 & 3 \\
\hline 44137 & HD 76909 & O93 & 7.843 & 0.452 & 0.263 & 0.407 & $<0.02$ & 7.843 & 0.452 & 0.263 & 0.407 & 0.38 & $\ldots$ & 1 & 1 \\
\hline 44892 & HD 78418 & O93 & 5.959 & 0.429 & 0.215 & 0.358 & $<0.02$ & 5.959 & 0.429 & 0.215 & 0.358 & -0.12 & $\ldots$ & 1 & 4 \\
\hline 46007 & HD 81110 & O93 & 8.298 & 0.435 & 0.247 & 0.366 & $<0.02$ & 8.298 & 0.435 & 0.247 & 0.366 & 0.24 & $\ldots$ & 1 & 15 \\
\hline 46580 & HD 82106 & O93 & 7.190 & 0.570 & 0.550 & 0.234 & $<0.02$ & 7.190 & 0.570 & 0.550 & 0.234 & 0.07 & 0.09 & 3 & $1,12,9$ \\
\hline 48133 & GJ 368.1A & O94 & 7.910 & 0.522 & 0.415 & 0.308 & $<0.02$ & 7.910 & 0.522 & 0.415 & 0.308 & 0.11 & $\ldots$ & 1 & 2 \\
\hline 48331 & HD 85512 & O93 & 7.627 & 0.660 & 0.666 & 0.176 & $<0.02$ & 7.627 & 0.660 & 0.666 & 0.176 & -0.10 & $\ldots$ & 1 & 13 \\
\hline 48423 & HD 85301 & O93 & 7.746 & 0.436 & 0.251 & 0.320 & $<0.02$ & 7.746 & 0.436 & 0.251 & 0.320 & 0.19 & $\ldots$ & 1 & 1 \\
\hline 49350 & HD 87359 & O93 & 7.495 & 0.429 & 0.224 & 0.340 & $<0.02$ & 7.495 & 0.429 & 0.224 & 0.340 & 0.06 & $\ldots$ & 1 & 1 \\
\hline 49680 & HD 87836 & O93 & 7.516 & 0.439 & 0.253 & 0.401 & $<0.02$ & 7.516 & 0.439 & 0.253 & 0.401 & 0.36 & $\ldots$ & 1 & 1 \\
\hline 49908 & HD 88230 & O93 & 6.553 & 0.790 & 0.741 & 0.030 & $<0.02$ & 6.553 & 0.790 & 0.741 & 0.030 & -0.10 & $\ldots$ & 1 & 14 \\
\hline 49942 & HD 88371 & SN88 & 8.414 & 0.407 & 0.186 & 0.329 & $<0.02$ & 8.414 & 0.407 & 0.186 & 0.329 & -0.31 & .. & 1 & 1 \\
\hline 50505 & HD 89269 & O93 & 6.656 & 0.420 & 0.208 & 0.292 & $<0.02$ & 6.656 & 0.420 & 0.208 & 0.292 & -0.21 & 0.01 & 2 & 1,9 \\
\hline 51257 & HD 90711 & O93 & 7.876 & 0.482 & 0.337 & 0.359 & $<0.02$ & 7.876 & 0.482 & 0.337 & 0.359 & 0.30 & $\ldots$ & 1 & 1 \\
\hline 51258 & HD 90722 & O93 & 7.875 & 0.445 & 0.271 & 0.388 & $<0.02$ & 7.875 & 0.445 & 0.271 & 0.388 & 0.36 & $\ldots$ & 1 & 1 \\
\hline 52409 & HD 92788 & O93 & 7.317 & 0.439 & 0.243 & 0.385 & $<0.02$ & 7.317 & 0.439 & 0.243 & 0.385 & 0.31 & 0.03 & 4 & $1,7,10,13$ \\
\hline
\end{tabular}


Table B.1. continued.

\begin{tabular}{|c|c|c|c|c|c|c|c|c|c|c|c|c|c|c|c|}
\hline HIP & Name & $\begin{array}{l}\text { Ref. } \\
\text { uvby }\end{array}$ & V & $(b-y)$ & $m_{1}$ & $c_{1}$ & $E(B-V)$ & $V_{0}$ & $(b-y)_{0}$ & $m_{1,0}$ & $c_{1,0}$ & $\langle[\mathrm{Fe} / \mathrm{H}]\rangle$ & $\begin{array}{r}{[\mathrm{Fe} / \mathrm{H}]} \\
\text { range }\end{array}$ & $\mathrm{N}$ & $\begin{array}{c}\text { Ref. } \\
{[\mathrm{Fe} / \mathrm{H}]}\end{array}$ \\
\hline 52462 & HD 92945 & O94 & 7.713 & 0.507 & 0.387 & 0.279 & $<0.02$ & 7.713 & 0.507 & 0.387 & 0.279 & 0.13 & $\ldots$ & 1 & 1 \\
\hline 54035 & HD 95735 & $\mathrm{O} 93$ & 7.422 & 0.981 & 0.419 & 0.155 & $<0.02$ & 7.422 & 0.981 & 0.419 & 0.155 & -0.67 & $\ldots$ & 1 & 14 \\
\hline 54287 & HD 96423 & O93 & 7.233 & 0.427 & 0.222 & 0.370 & $<0.02$ & 7.233 & 0.427 & 0.222 & 0.370 & 0.10 & $\ldots$ & 1 & 1 \\
\hline 54538 & GJ 9350 & $\mathrm{O} 93$ & 9.728 & 0.471 & 0.286 & 0.286 & $<0.02$ & 9.728 & 0.471 & 0.286 & 0.286 & -0.34 & $\ldots$ & 1 & 2 \\
\hline 54651 & BD -10 3216 & O93 & 9.192 & 0.636 & 0.559 & 0.135 & $<0.02$ & 9.192 & 0.636 & 0.559 & 0.135 & -1.14 & $\ldots$ & 1 & 8 \\
\hline 54704 & HD 97343 & SN88 & 7.052 & 0.460 & 0.296 & 0.324 & $<0.02$ & 7.052 & 0.460 & 0.296 & 0.324 & -0.04 & $\ldots$ & 1 & 1 \\
\hline 55013 & HD 97998 & SN88 & 7.362 & 0.397 & 0.185 & 0.260 & $<0.02$ & 7.362 & 0.397 & 0.185 & 0.260 & -0.41 & $\ldots$ & 1 & 1 \\
\hline 55022 & HD 97916 & SN88 & 9.209 & 0.293 & 0.104 & 0.407 & 0.025 & 9.126 & 0.273 & 0.111 & 0.403 & -1.06 & $\ldots$ & 1 & 4 \\
\hline 55210 & HD 98281 & $\mathrm{O} 93$ & 7.272 & 0.457 & 0.254 & 0.288 & $<0.02$ & 7.272 & 0.457 & 0.254 & 0.288 & -0.20 & $\ldots$ & 1 & 1 \\
\hline 55846 & HD 99491 & O93 & 6.488 & 0.484 & 0.335 & 0.362 & $<0.02$ & 6.488 & 0.484 & 0.335 & 0.362 & 0.34 & $\ldots$ & 1 & 1 \\
\hline 55848 & HD 99492 & O93 & 7.550 & 0.578 & 0.579 & 0.262 & $<0.02$ & 7.550 & 0.578 & 0.579 & 0.262 & 0.33 & 0.05 & 3 & $, 2,13$ \\
\hline 55900 & HD 99610 & O93 & 7.414 & 0.436 & 0.239 & 0.377 & $<0.02$ & 7.414 & 0.436 & 0.239 & 0.377 & 0.23 & $\ldots$ & 1 & 1 \\
\hline 56242 & HD 100180 & O93 & 9.146 & 0.652 & 0.669 & 0.131 & $<0.02$ & 9.146 & 0.652 & 0.669 & 0.131 & -0.00 & 0.02 & 2 & 1,4 \\
\hline 56452 & HD 100623 & O93 & 5.955 & 0.485 & 0.320 & 0.241 & $<0.02$ & 5.955 & 0.485 & 0.320 & 0.241 & -0.38 & 0.02 & 2 & 1,13 \\
\hline 56830 & HD 101259 & O93 & 6.409 & 0.508 & 0.229 & 0.315 & $<0.02$ & 6.409 & 0.508 & 0.229 & 0.315 & -0.69 & $\ldots$ & 1 & 1 \\
\hline 56832 & HD 101242 & O93 & 7.611 & 0.412 & 0.226 & 0.327 & $<0.02$ & 7.611 & 0.412 & 0.226 & 0.327 & 0.08 & $\ldots$ & 1 & 3 \\
\hline 56998 & HD 101581 & O93 & 7.751 & 0.602 & 0.580 & 0.175 & $<0.02$ & 7.751 & 0.602 & 0.580 & 0.175 & -0.32 & $\ldots$ & 1 & 13 \\
\hline 57001 & HD 101563 & O94 & 6.436 & 0.404 & 0.212 & 0.334 & $<0.02$ & 6.436 & 0.404 & 0.212 & 0.334 & 0.04 & $\ldots$ & 1 & 7 \\
\hline 57271 & HD 102071 & O94 & 7.973 & 0.475 & 0.315 & 0.309 & $<0.02$ & 7.973 & 0.475 & 0.315 & 0.309 & 0.01 & $\ldots$ & 1 & 1 \\
\hline 57291 & HD 102117 & O93 & 7.462 & 0.455 & 0.246 & 0.407 & $<0.02$ & 7.462 & 0.455 & 0.246 & 0.407 & 0.30 & 0.01 & 2 & 1,13 \\
\hline 57349 & HD 102158 & SN88 & 8.066 & 0.393 & 0.163 & 0.309 & $<0.02$ & 8.066 & 0.393 & 0.163 & 0.309 & -0.47 & $\ldots$ & 1 & 1 \\
\hline 57443 & HD 102365 & O93 & 4.892 & 0.418 & 0.199 & 0.278 & $<0.02$ & 4.892 & 0.418 & 0.199 & 0.278 & -0.32 & 0.02 & 2 & 1,13 \\
\hline 57450 & $\mathrm{BD}+511696$ & SN88 & 9.912 & 0.397 & 0.100 & 0.180 & $<0.02$ & 9.912 & 0.397 & 0.100 & 0.180 & -1.48 & $\ldots$ & 1 & 1 \\
\hline 57507 & HD 102438 & O93 & 6.478 & 0.433 & 0.210 & 0.281 & $<0.02$ & 6.478 & 0.433 & 0.210 & 0.281 & -0.27 & 0.01 & 2 & 1,13 \\
\hline 57939 & HD 103095 & SN88 & 6.416 & 0.484 & 0.219 & 0.167 & $<0.02$ & 6.416 & 0.484 & 0.219 & 0.167 & -1.28 & 0.14 & 3 & $1,7,12$ \\
\hline 58067 & HD 103432 & O93 & 8.211 & 0.420 & 0.224 & 0.287 & $<0.02$ & 8.211 & 0.420 & 0.224 & 0.287 & -0.10 & 0.05 & 2 & 1,2 \\
\hline 58345 & HD 103932 & O93 & 6.949 & 0.630 & 0.709 & 0.162 & $<0.02$ & 6.949 & 0.630 & 0.709 & 0.162 & 0.15 & 0.16 & 4 & $3,12,13,5$ \\
\hline 58576 & HD 104304 & O93 & 5.546 & 0.464 & 0.319 & 0.335 & $<0.02$ & 5.546 & 0.464 & 0.319 & 0.335 & 0.30 & 0.09 & 3 & $1,12,13$ \\
\hline 58843 & HD 104800 & SN88 & 9.227 & 0.382 & 0.141 & 0.255 & $<0.02$ & 9.227 & 0.382 & 0.141 & 0.255 & -0.82 & $\ldots$ & 1 & 1 \\
\hline 59572 & HD 106156 & $\mathrm{O} 93$ & 7.917 & 0.471 & 0.329 & 0.341 & $<0.02$ & 7.917 & 0.471 & 0.329 & 0.341 & 0.19 & 0.09 & 2 & 1,3 \\
\hline 59750 & HD 106516 & SN88 & 6.111 & 0.318 & 0.110 & 0.335 & $<0.02$ & 6.111 & 0.318 & 0.110 & 0.335 & -0.80 & 0.08 & 2 & 4,9 \\
\hline 60081 & HD 107148 & O93 & 8.022 & 0.428 & 0.238 & 0.404 & $<0.02$ & 8.022 & 0.428 & 0.238 & 0.404 & 0.31 & $\ldots$ & 1 & 1 \\
\hline 60729 & HD 108309 & O93 & 8.231 & 0.437 & 0.164 & 0.366 & $<0.02$ & 8.231 & 0.437 & 0.164 & 0.366 & 0.13 & $\ldots$ & 1 & 1 \\
\hline 61028 & HD 108874 & O93 & 8.764 & 0.457 & 0.277 & 0.356 & $<0.02$ & 8.764 & 0.457 & 0.277 & 0.356 & 0.21 & 0.05 & 2 & 1,10 \\
\hline 61291 & HD 109200 & O93 & 7.137 & 0.496 & 0.351 & 0.272 & $<0.02$ & 7.137 & 0.496 & 0.351 & 0.272 & -0.26 & 0.07 & 2 & 1,13 \\
\hline 61595 & HD 109749 & $\mathrm{O} 93$ & 8.176 & 0.425 & 0.207 & 0.395 & $<0.02$ & 8.176 & 0.425 & 0.207 & 0.395 & 0.26 & $\ldots$ & 1 & 15 \\
\hline 62145 & HD 110833 & O93 & 7.014 & 0.548 & 0.477 & 0.286 & $<0.02$ & 7.014 & 0.548 & 0.477 & 0.286 & 0.15 & $\ldots$ & 1 & 9 \\
\hline 62345 & HD 111031 & O93 & 6.890 & 0.426 & 0.250 & 0.376 & $<0.02$ & 6.890 & 0.426 & 0.250 & 0.376 & 0.28 & $\ldots$ & 1 & 1 \\
\hline 62523 & HD 111395 & O93 & 6.288 & 0.438 & 0.241 & 0.334 & $<0.02$ & 6.288 & 0.438 & 0.241 & 0.334 & 0.11 & 0.04 & 2 & 1,7 \\
\hline 62534 & HD 111232 & $\mathrm{O} 93$ & 7.598 & 0.436 & 0.211 & 0.273 & $<0.02$ & 7.598 & 0.436 & 0.211 & 0.273 & -0.36 & $\ldots$ & 1 & 10 \\
\hline 62536 & HD 111398 & O93 & 7.105 & 0.425 & 0.213 & 0.368 & $<0.02$ & 7.105 & 0.425 & 0.213 & 0.368 & 0.08 & $\ldots$ & 1 & 1 \\
\hline 62607 & HD 111515 & O93 & 8.113 & 0.437 & 0.201 & 0.241 & $<0.02$ & 8.113 & 0.437 & 0.201 & 0.241 & -0.60 & $\ldots$ & 1 & 1 \\
\hline 63048 & HD 112257 & O93 & 7.809 & 0.423 & 0.213 & 0.336 & $<0.02$ & 7.809 & 0.423 & 0.213 & 0.336 & -0.03 & $\ldots$ & 1 & 1 \\
\hline 64150 & HD 114174 & O93 & 6.794 & 0.423 & 0.217 & 0.340 & $<0.02$ & 6.794 & 0.423 & 0.217 & 0.340 & 0.07 & $\ldots$ & 1 & 1 \\
\hline 64219 & HD 114260 & O93 & 7.356 & 0.452 & 0.248 & 0.310 & $<0.02$ & 7.356 & 0.452 & 0.248 & 0.310 & -0.09 & $\ldots$ & 1 & 1 \\
\hline 64275 & BD +68 714 & O93 & 8.868 & 0.542 & 0.460 & 0.287 & $<0.02$ & 8.868 & 0.542 & 0.460 & 0.287 & 0.22 & $\ldots$ & 1 & 8 \\
\hline 64408 & HD 114613 & O93 & 4.848 & 0.441 & 0.235 & 0.390 & $<0.02$ & 4.848 & 0.441 & 0.235 & 0.390 & 0.24 & $\ldots$ & 1 & 1 \\
\hline 64426 & HD 114762 & SN88 & 7.324 & 0.366 & 0.123 & 0.300 & $<0.02$ & 7.324 & 0.366 & 0.123 & 0.300 & -0.68 & 0.07 & 3 & $1,7,10$ \\
\hline 64457 & HD 114783 & O84 & 7.565 & 0.521 & 0.458 & 0.309 & $<0.02$ & 7.565 & 0.521 & 0.458 & 0.309 & 0.13 & 0.02 & 2 & 1,10 \\
\hline 64459 & HD 114729 & SN88 & 6.687 & 0.391 & 0.163 & 0.344 & $<0.02$ & 6.687 & 0.391 & 0.163 & 0.344 & -0.27 & 0.01 & 2 & 1,10 \\
\hline 64577 & HD 114946 & O93 & 5.321 & 0.523 & 0.322 & 0.317 & $<0.02$ & 5.321 & 0.523 & 0.322 & 0.317 & -0.21 & $\ldots$ & 1 & 1 \\
\hline 64924 & HD 115617 & O93 & 4.734 & 0.433 & 0.256 & 0.328 & $<0.02$ & 4.734 & 0.433 & 0.256 & 0.328 & 0.03 & 0.05 & 3 & $1,12,13$ \\
\hline 64965 & LHS 2715 & SN88 & 10.803 & 0.587 & 0.517 & 0.115 & $<0.02$ & 10.803 & 0.587 & 0.517 & 0.115 & -1.46 & 0.03 & 2 & 8,14 \\
\hline 65036 & HD 115585 & O94 & 7.432 & 0.457 & 0.260 & 0.407 & $<0.02$ & 7.432 & 0.457 & 0.260 & 0.407 & 0.34 & $\ldots$ & 1 & 1 \\
\hline 65352 & HD 116442 & O93 & 7.053 & 0.467 & 0.310 & 0.251 & $<0.02$ & 7.053 & 0.467 & 0.310 & 0.251 & -0.40 & $\ldots$ & 1 & 1 \\
\hline 65355 & HD 116443 & O93 & 7.343 & 0.493 & 0.369 & 0.260 & $<0.02$ & 7.343 & 0.493 & 0.369 & 0.260 & -0.36 & 0.04 & 2 & 1,9 \\
\hline 65530 & HD 117043 & $\mathrm{O} 93$ & 6.510 & 0.458 & 0.272 & 0.352 & $<0.02$ & 6.510 & 0.458 & 0.272 & 0.352 & 0.29 & $\ldots$ & 1 & 9 \\
\hline 65708 & HD 117126 & O93 & 7.432 & 0.417 & 0.200 & 0.347 & $<0.02$ & 7.432 & 0.417 & 0.200 & 0.347 & -0.03 & $\ldots$ & 1 & 1 \\
\hline 65721 & HD 117176 & O93 & 4.965 & 0.451 & 0.221 & 0.354 & $<0.02$ & 4.965 & 0.451 & 0.221 & 0.354 & 0.00 & 0.09 & 4 & $1,7,9,10$ \\
\hline 65808 & HD 117207 & O93 & 7.248 & 0.451 & 0.255 & 0.358 & $<0.02$ & 7.248 & 0.451 & 0.255 & 0.358 & 0.24 & 0.05 & 2 & 1,13 \\
\hline 65982 & HD 117635 & O93 & 7.325 & 0.474 & 0.284 & 0.249 & $<0.02$ & 7.325 & 0.474 & 0.284 & 0.249 & -0.40 & $\ldots$ & 1 & 9 \\
\hline 66238 & HD 117939 & O93 & 7.284 & 0.424 & 0.191 & 0.303 & $<0.02$ & 7.284 & 0.424 & 0.191 & 0.303 & -0.22 & 0.03 & 2 & 1,2 \\
\hline 66618 & HD 118475 & SN88 & 6.976 & 0.386 & 0.206 & 0.372 & $<0.02$ & 6.976 & 0.386 & 0.206 & 0.372 & 0.10 & $\ldots$ & 1 & 1 \\
\hline 66765 & HD 118972 & $\mathrm{O} 93$ & 6.922 & 0.504 & 0.363 & 0.287 & $<0.02$ & 6.922 & 0.504 & 0.363 & 0.287 & 0.00 & $\ldots$ & 2 & 1,13 \\
\hline 67408 & HD 120237 & SN88 & 6.584 & 0.355 & 0.167 & 0.364 & $<0.02$ & 6.584 & 0.355 & 0.167 & 0.364 & -0.03 & $\ldots$ & 1 & 1 \\
\hline 67487 & HD 120467 & O93 & 8.152 & 0.700 & 0.785 & 0.079 & $<0.02$ & 8.152 & 0.700 & 0.785 & 0.079 & 0.02 & $\cdots$ & 1 & 12 \\
\hline
\end{tabular}


Table B.1. continued.

\begin{tabular}{|c|c|c|c|c|c|c|c|c|c|c|c|c|c|c|c|}
\hline HIP & Name & $\begin{array}{l}\text { Ref. } \\
\text { uvby }\end{array}$ & V & $(b-y)$ & $m_{1}$ & $c_{1}$ & $E(B-V)$ & $V_{0}$ & $(b-y)_{0}$ & $m_{1,0}$ & $c_{1,0}$ & $\langle[\mathrm{Fe} / \mathrm{H}]\rangle$ & $\begin{array}{r}{[\mathrm{Fe} / \mathrm{H}]} \\
\text { range }\end{array}$ & $\mathrm{N}$ & $\begin{array}{c}\text { Ref. } \\
{[\mathrm{Fe} / \mathrm{H}]}\end{array}$ \\
\hline 67620 & HD 120690 & O93 & 6.442 & 0.433 & 0.236 & 0.315 & $<0.02$ & 6.442 & 0.433 & 0.236 & 0.315 & -0.03 & 0.16 & 2 & 1,2 \\
\hline 68273 & HD 121849 & O93 & 8.155 & 0.432 & 0.202 & 0.294 & $<0.02$ & 8.155 & 0.432 & 0.202 & 0.294 & -0.29 & $\ldots$ & 1 & 2 \\
\hline 68634 & HD 122676 & O93 & 7.127 & 0.458 & 0.242 & 0.314 & $<0.02$ & 7.127 & 0.458 & 0.242 & 0.314 & -0.02 & $\ldots$ & 1 & 1 \\
\hline 68682 & HD 122742 & O93 & 6.273 & 0.452 & 0.264 & 0.317 & $<0.02$ & 6.273 & 0.452 & 0.264 & 0.317 & 0.01 & $\ldots$ & 1 & 1 \\
\hline 69357 & HD 124106 & O93 & 7.924 & 0.515 & 0.370 & 0.285 & $<0.02$ & 7.924 & 0.515 & 0.370 & 0.285 & -0.10 & $\ldots$ & 1 & 1 \\
\hline 69390 & HD 124244 & O93 & 8.467 & 0.420 & 0.195 & 0.379 & $<0.02$ & 8.467 & 0.420 & 0.195 & 0.379 & 0.20 & $\ldots$ & 1 & 4 \\
\hline 69881 & HD 125184 & O93 & 6.466 & 0.454 & 0.247 & 0.413 & $<0.02$ & 6.466 & 0.454 & 0.247 & 0.413 & 0.34 & 0.12 & 3 & $1,9,15$ \\
\hline 69972 & HD 125072 & O93 & 6.642 & 0.583 & 0.594 & 0.230 & $<0.02$ & 6.642 & 0.583 & 0.594 & 0.230 & 0.30 & 0.03 & 2 & 1,13 \\
\hline 70016 & HD 125455 & O93 & 7.577 & 0.494 & 0.374 & 0.285 & $<0.02$ & 7.577 & 0.494 & 0.374 & 0.285 & -0.15 & 0.05 & 2 & 1,11 \\
\hline 70470 & HD 126511 & O93 & 8.365 & 0.460 & 0.301 & 0.321 & $<0.02$ & 8.365 & 0.460 & 0.301 & 0.321 & 0.09 & $\ldots$ & 1 & 3 \\
\hline 70873 & HD 127334 & O93 & 6.362 & 0.441 & 0.245 & 0.366 & $<0.02$ & 6.362 & 0.441 & 0.245 & 0.366 & 0.27 & $\ldots$ & 1 & 1 \\
\hline 71462 & HD 128428 & O93 & 7.803 & 0.466 & 0.277 & 0.412 & 0.026 & 7.717 & 0.446 & 0.284 & 0.407 & 0.47 & $\ldots$ & 1 & 1 \\
\hline 71735 & HD 128674 & O93 & 7.393 & 0.423 & 0.204 & 0.265 & $<0.02$ & 7.393 & 0.423 & 0.204 & 0.265 & -0.39 & $\ldots$ & 1 & 1 \\
\hline 71743 & HD 128987 & O93 & 7.238 & 0.439 & 0.269 & 0.304 & $<0.02$ & 7.238 & 0.439 & 0.269 & 0.304 & 0.04 & $\ldots$ & 1 & 3 \\
\hline 72312 & HD 130307 & O93 & 7.776 & 0.521 & 0.405 & 0.275 & $<0.02$ & 7.776 & 0.521 & 0.405 & 0.275 & -0.16 & $\ldots$ & 1 & 1 \\
\hline 72339 & HD 130322 & O93 & 8.036 & 0.475 & 0.305 & 0.316 & $<0.02$ & 8.036 & 0.475 & 0.305 & 0.316 & 0.04 & 0.07 & 3 & $1,7,10$ \\
\hline 72577 & HD 130871 & O93 & 9.060 & 0.545 & 0.507 & 0.231 & $<0.02$ & 9.060 & 0.545 & 0.507 & 0.231 & -0.16 & 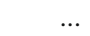 & 1 & 1 \\
\hline 72688 & HD 130992 & O93 & 7.790 & 0.568 & 0.556 & 0.233 & $<0.02$ & 7.790 & 0.568 & 0.556 & 0.233 & -0.00 & 0.01 & 2 & 1,13 \\
\hline 72875 & BD +23 2751 & O93 & 8.621 & 0.554 & 0.498 & 0.212 & $<0.02$ & 8.621 & 0.554 & 0.498 & 0.212 & -0.34 & $\ldots$ & 1 & 8 \\
\hline 73005 & HD 132142 & O93 & 7.756 & 0.482 & 0.297 & 0.267 & $<0.02$ & 7.756 & 0.482 & 0.297 & 0.267 & -0.45 & $\ldots$ & 1 & 1 \\
\hline 73182 & HD 131976 & O93 & 8.052 & 0.944 & 0.486 & 0.189 & $<0.02$ & 8.052 & 0.944 & 0.486 & 0.189 & 0.07 & 0.16 & 6 & $1,3,12,9,13,5$ \\
\hline 73184 & HD 131977 & O93 & 5.725 & 0.609 & 0.660 & 0.187 & $<0.02$ & 5.725 & 0.609 & 0.660 & 0.187 & 0.07 & 0.16 & 6 & $1,3,12,9,13,5$ \\
\hline 73241 & HD 131923 & O93 & 6.346 & 0.443 & 0.228 & 0.361 & $<0.02$ & 6.346 & 0.443 & 0.228 & 0.361 & 0.11 & $\ldots$ & 1 & 1 \\
\hline 73869 & HD 134319 & O93 & 8.425 & 0.419 & 0.222 & 0.276 & $<0.02$ & 8.425 & 0.419 & 0.222 & 0.276 & 0.03 & $\ldots$ & 1 & 1 \\
\hline 74135 & HD 134474 & O93 & 8.880 & 0.482 & 0.376 & 0.318 & $<0.02$ & 8.880 & 0.482 & 0.376 & 0.318 & 0.14 & $\ldots$ & 1 & 3 \\
\hline 74235 & HIP 74235 & O93 & 9.058 & 0.487 & 0.216 & 0.159 & $<0.02$ & 9.058 & 0.487 & 0.216 & 0.159 & -1.45 & 0.06 & 2 & 1,8 \\
\hline 74432 & HD 135101 & O93 & 6.675 & 0.436 & 0.217 & 0.369 & $<0.02$ & 6.675 & 0.436 & 0.217 & 0.369 & 0.08 & $\ldots$ & 1 & 1 \\
\hline 74434 & HD 135101 & O93 & 7.518 & 0.460 & 0.251 & 0.353 & $<0.02$ & 7.518 & 0.460 & 0.251 & 0.353 & 0.08 & $\ldots$ & 1 & 1 \\
\hline 74500 & HD 134987 & O93 & 6.468 & 0.434 & 0.256 & 0.375 & $<0.02$ & 6.468 & 0.434 & 0.256 & 0.375 & 0.31 & 0.08 & 4 & $1,3,7,10$ \\
\hline 75181 & HD 136352 & SN88 & 5.654 & 0.401 & 0.198 & 0.291 & $<0.02$ & 5.654 & 0.401 & 0.198 & 0.291 & -0.34 & 0.01 & 2 & 1,13 \\
\hline 75266 & HD 136834 & O93 & 8.255 & 0.560 & 0.563 & 0.241 & $<0.02$ & 8.255 & 0.560 & 0.563 & 0.241 & 0.27 & 0.03 & 3 & $1,3,5$ \\
\hline 75676 & HD 138004 A & O93 & 7.496 & 0.415 & 0.244 & 0.202 & $<0.02$ & 7.496 & 0.415 & 0.244 & 0.202 & -0.09 & $\ldots$ & 1 & 1 \\
\hline 75676 & HD 138004 B & O93 & 9.831 & 0.688 & 0.699 & 0.099 & $<0.02$ & 9.831 & 0.688 & 0.699 & 0.099 & -0.09 & $\ldots$ & 1 & 1 \\
\hline 75722 & HD 137778 & O93 & 7.567 & 0.532 & 0.455 & 0.286 & $<0.02$ & 7.567 & 0.532 & 0.455 & 0.286 & 0.28 & $\ldots$ & 1 & 1 \\
\hline 75829 & HD 139813 & O93 & 7.356 & 0.481 & 0.298 & 0.292 & $<0.02$ & 7.356 & 0.481 & 0.298 & 0.292 & 0.14 & $\ldots$ & 1 & 1 \\
\hline 76200 & HD 138549 & O93 & 7.962 & 0.438 & 0.264 & 0.310 & $<0.02$ & 7.962 & 0.438 & 0.264 & 0.310 & 0.00 & $\ldots$ & 1 & 1 \\
\hline 78170 & HD 142709 & O93 & 8.026 & 0.624 & 0.641 & 0.173 & $<0.02$ & 8.026 & 0.624 & 0.641 & 0.173 & -0.12 & $\ldots$ & 1 & 13 \\
\hline 78241 & HD 143291 & O93 & 8.003 & 0.465 & 0.274 & 0.252 & $<0.02$ & 8.003 & 0.465 & 0.274 & 0.252 & -0.44 & $\ldots$ & 1 & 1 \\
\hline 78709 & HD 144287 & O93 & 7.094 & 0.470 & 0.270 & 0.325 & $<0.02$ & 7.094 & 0.470 & 0.270 & 0.325 & -0.09 & $\ldots$ & 1 & 9 \\
\hline 78775 & HD 144579 & O93 & 6.665 & 0.455 & 0.232 & 0.226 & $<0.02$ & 6.665 & 0.455 & 0.232 & 0.226 & -0.68 & 0.02 & 3 & $1,12,9$ \\
\hline 78843 & HD 144253 & O93 & 7.379 & 0.590 & 0.595 & 0.206 & $<0.02$ & 7.379 & 0.590 & 0.595 & 0.206 & -0.04 & $\ldots$ & 1 & 2 \\
\hline 79143 & HD 144009 & O93 & 7.232 & 0.450 & 0.251 & 0.327 & $<0.02$ & 7.232 & 0.450 & 0.251 & 0.327 & 0.06 & $\ldots$ & 1 & 1 \\
\hline 79190 & HD 144628 & O94 & 7.113 & 0.494 & 0.363 & 0.254 & $<0.02$ & 7.113 & 0.494 & 0.363 & 0.254 & -0.37 & 0.06 & 3 & $1,2,13$ \\
\hline 79242 & HD 142022 & O94 & 11.054 & 0.821 & 0.691 & 0.079 & $<0.02$ & 11.054 & 0.821 & 0.691 & 0.079 & 0.19 & $\ldots$ & 1 & 13 \\
\hline 79242 & HD 142022 & O94 & 7.725 & 0.464 & 0.287 & 0.364 & $<0.02$ & 7.725 & 0.464 & 0.287 & 0.364 & 0.19 & $\ldots$ & 1 & 13 \\
\hline 79537 & HD 145417 & O93 & 7.522 & 0.504 & 0.280 & 0.162 & $<0.02$ & 7.522 & 0.504 & 0.280 & 0.162 & -1.13 & $\ldots$ & 1 & 13 \\
\hline 79619 & HD 147231 & O93 & 7.836 & 0.443 & 0.217 & 0.328 & $<0.02$ & 7.836 & 0.443 & 0.217 & 0.328 & 0.00 & $\ldots$ & 1 & 1 \\
\hline 79967 & HD 146481 & O93 & 7.097 & 0.418 & 0.159 & 0.319 & $<0.02$ & 7.097 & 0.418 & 0.159 & 0.319 & -0.44 & $\ldots$ & 1 & 1 \\
\hline 81022 & HD 149143 & O93 & 7.899 & 0.415 & 0.206 & 0.424 & $<0.02$ & 7.899 & 0.415 & 0.206 & 0.424 & 0.38 & $\ldots$ & 1 & 15 \\
\hline 81210 & HD 149750 & O93 & 8.588 & 0.418 & 0.209 & 0.379 & $<0.02$ & 8.588 & 0.418 & 0.209 & 0.379 & 0.24 & $\ldots$ & 1 & 4 \\
\hline 81294 & BD -14 4454 & O93 & 10.332 & 0.557 & 0.461 & 0.188 & 0.092 & 10.031 & 0.486 & 0.486 & 0.172 & -0.85 & $\ldots$ & 1 & 8 \\
\hline 81300 & HD 149661 & O93 & 5.766 & 0.491 & 0.366 & 0.297 & $<0.02$ & 5.766 & 0.491 & 0.366 & 0.297 & 0.06 & 0.04 & 5 & $1,7,12,9,13$ \\
\hline 81347 & HD 149724 & O93 & 7.854 & 0.460 & 0.267 & 0.398 & 0.060 & 7.657 & 0.414 & 0.283 & 0.387 & 0.41 & $\ldots$ & 1 & 1 \\
\hline 81746 & HD 150248 & O93 & 7.026 & 0.418 & 0.202 & 0.323 & $<0.02$ & 7.026 & 0.418 & 0.202 & 0.323 & -0.11 & $\ldots$ & 1 & 1 \\
\hline 81767 & HD 150437 & O93 & 7.853 & 0.426 & 0.245 & 0.389 & 0.024 & 7.776 & 0.408 & 0.251 & 0.385 & 0.30 & $\ldots$ & 1 & 1 \\
\hline 81813 & HD 151541 & O93 & 7.640 & 0.469 & 0.280 & 0.281 & $<0.02$ & 7.640 & 0.469 & 0.280 & 0.281 & -0.16 & 0.05 & 2 & 1,9 \\
\hline 81819 & HD 150474 & O93 & 7.162 & 0.477 & 0.261 & 0.364 & 0.034 & 7.050 & 0.451 & 0.270 & 0.358 & 0.05 & $\ldots$ & 1 & 1 \\
\hline 81935 & HD 150689 & O94 & 7.511 & 0.574 & 0.565 & 0.221 & $<0.02$ & 7.511 & 0.574 & 0.565 & 0.221 & -0.03 & $\ldots$ & 1 & 13 \\
\hline 82588 & HD 152391 & O93 & 6.608 & 0.456 & 0.285 & 0.298 & $<0.02$ & 6.608 & 0.456 & 0.285 & 0.298 & 0.02 & 0.04 & 3 & $1,9,13$ \\
\hline 82636 & HD 152792 & O93 & 6.818 & 0.415 & 0.158 & 0.325 & $<0.02$ & 6.818 & 0.415 & 0.158 & 0.325 & -0.31 & $\ldots$ & 1 & 1 \\
\hline 83229 & HD 153075 & SN88 & 7.011 & 0.389 & 0.144 & 0.298 & $<0.02$ & 7.011 & 0.389 & 0.144 & 0.298 & -0.55 & $\ldots$ & 1 & 1 \\
\hline 83906 & HD 154962 & O93 & 6.353 & 0.440 & 0.235 & 0.409 & 0.048 & 6.195 & 0.403 & 0.248 & 0.401 & 0.32 & 0.03 & 2 & 1,15 \\
\hline 83990 & HD 154577 & O94 & 7.385 & 0.510 & 0.391 & 0.224 & $<0.02$ & 7.385 & 0.510 & 0.391 & 0.224 & -0.66 & 0.06 & 2 & 1,13 \\
\hline 84489 & HD 155974 & SN88 & 6.094 & 0.321 & 0.143 & 0.407 & $<0.02$ & 6.094 & 0.321 & 0.143 & 0.407 & -0.17 & $\ldots$ & 1 & 1 \\
\hline 84636 & HD 156365 & O93 & 6.603 & 0.418 & 0.230 & 0.406 & 0.051 & 6.436 & 0.379 & 0.244 & 0.397 & 0.28 & 0.03 & 2 & 1,15 \\
\hline 84801 & HD 156826 & O93 & 6.316 & 0.519 & 0.296 & 0.327 & 0.079 & 6.056 & 0.458 & 0.317 & 0.313 & -0.13 & $\ldots$ & 1 & 1 \\
\hline
\end{tabular}


Table B.1. continued.

\begin{tabular}{|c|c|c|c|c|c|c|c|c|c|c|c|c|c|c|c|}
\hline HIP & Name & $\begin{array}{l}\text { Ref. } \\
\text { uvby }\end{array}$ & V & $(b-y)$ & $m_{1}$ & $c_{1}$ & $E(B-V)$ & $V_{0}$ & $(b-y)_{0}$ & $m_{1,0}$ & $c_{1,0}$ & $\langle[\mathrm{Fe} / \mathrm{H}]\rangle$ & $\begin{array}{r}{[\mathrm{Fe} / \mathrm{H}]} \\
\text { range }\end{array}$ & $\mathrm{N}$ & $\begin{array}{c}\text { Ref. } \\
{[\mathrm{Fe} / \mathrm{H}]}\end{array}$ \\
\hline 84905 & HD 157089 & SN88 & 6.961 & 0.379 & 0.139 & 0.326 & 0.023 & 6.886 & 0.361 & 0.145 & 0.322 & -0.57 & $\ldots$ & 1 & 9 \\
\hline 84988 & HD 155918 & SN88 & 7.012 & 0.389 & 0.145 & 0.271 & $<0.02$ & 7.012 & 0.389 & 0.145 & 0.271 & -0.64 & $\ldots$ & 1 & 1 \\
\hline 85017 & HD 157172 & O93 & 7.848 & 0.466 & 0.298 & 0.350 & 0.028 & 7.756 & 0.444 & 0.306 & 0.345 & 0.17 & $\ldots$ & 1 & 1 \\
\hline 85042 & HD 157347 & $\mathrm{O} 93$ & 6.293 & 0.426 & 0.221 & 0.349 & 0.025 & 6.213 & 0.407 & 0.228 & 0.345 & 0.08 & 0.10 & 2 & 1,4 \\
\hline 85235 & HD 158633 & O93 & 6.429 & 0.466 & 0.271 & 0.240 & $<0.02$ & 6.429 & 0.466 & 0.271 & 0.240 & -0.43 & 0.06 & 3 & $1,12,9$ \\
\hline 85653 & HD 159062 & O93 & 7.215 & 0.458 & 0.258 & 0.238 & $<0.02$ & 7.215 & 0.458 & 0.258 & 0.238 & -0.36 & $\ldots$ & 1 & 9 \\
\hline 85969 & HD 158783 & O93 & 7.096 & 0.424 & 0.209 & 0.366 & $<0.02$ & 7.096 & 0.424 & 0.209 & 0.366 & 0.13 & $\ldots$ & 1 & 1 \\
\hline 86013 & HD 159482 & SN88 & 8.387 & 0.382 & 0.126 & 0.277 & $<0.02$ & 8.387 & 0.382 & 0.126 & 0.277 & -0.93 & $\ldots$ & 1 & 9 \\
\hline 86375 & HD 159868 & $\mathrm{O} 93$ & 7.230 & 0.451 & 0.219 & 0.356 & 0.026 & 7.146 & 0.431 & 0.226 & 0.351 & 0.00 & $\ldots$ & 1 & 1 \\
\hline 86400 & HD 160346 & O93 & 6.501 & 0.551 & 0.500 & 0.261 & $<0.02$ & 6.501 & 0.551 & 0.500 & 0.261 & 0.01 & 0.10 & 2 & 12,9 \\
\hline 86765 & HD 161098 & O93 & 7.671 & 0.425 & 0.215 & 0.282 & 0.031 & 7.568 & 0.401 & 0.223 & 0.276 & -0.24 & $\ldots$ & 1 & 9 \\
\hline 86796 & HD 160691 & O93 & 5.123 & 0.432 & 0.244 & 0.395 & $<0.02$ & 5.123 & 0.432 & 0.244 & 0.395 & 0.30 & 0.03 & 4 & $2,10,13$ \\
\hline 86974 & HD 161797 & O93 & 3.418 & 0.474 & 0.259 & 0.398 & $<0.02$ & 3.418 & 0.474 & 0.259 & 0.398 & 0.28 & 0.04 & 2 & 1,12 \\
\hline 86985 & HD 161555 & O93 & 7.353 & 0.414 & 0.200 & 0.399 & 0.082 & 7.084 & 0.351 & 0.222 & 0.385 & 0.13 & $\ldots$ & 1 & 1 \\
\hline 87062 & BD -8 4501 & O93 & 10.586 & 0.447 & 0.042 & 0.270 & 0.124 & 10.178 & 0.351 & 0.075 & 0.248 & -1.47 & $\ldots$ & 2 & 1,8 \\
\hline 87089 & HD 161848 & O93 & 8.900 & 0.493 & 0.326 & 0.285 & 0.024 & 8.821 & 0.474 & 0.333 & 0.281 & -0.39 & $\ldots$ & 1 & 1 \\
\hline 87116 & HD 161612 & O93 & 7.186 & 0.447 & 0.246 & 0.375 & $<0.02$ & 7.186 & 0.447 & 0.246 & 0.375 & 0.14 & $\ldots$ & 1 & 1 \\
\hline 87330 & HD 162020 & O94 & 9.084 & 0.579 & 0.534 & 0.244 & $<0.02$ & 9.084 & 0.579 & 0.534 & 0.244 & 0.06 & 0.08 & 3 & $1,6,10$ \\
\hline 87710 & HD 163153 & O93 & 6.923 & 0.478 & 0.281 & 0.411 & 0.058 & 6.732 & 0.433 & 0.297 & 0.401 & 0.49 & - & 1 & 1 \\
\hline 88194 & HD 164595 & O93 & 7.070 & 0.411 & 0.199 & 0.314 & $<0.02$ & 7.070 & 0.411 & 0.199 & 0.314 & -0.04 & 0.05 & 2 & 1,11 \\
\hline 88217 & HD 164507 & $\mathrm{O} 93$ & 6.284 & 0.463 & 0.230 & 0.402 & $<0.02$ & 6.284 & 0.463 & 0.230 & 0.402 & 0.19 & $\ldots$ & 1 & 1 \\
\hline 88348 & HD 164922 & O93 & 6.997 & 0.487 & 0.307 & 0.394 & $<0.02$ & 6.997 & 0.487 & 0.307 & 0.394 & 0.16 & 0.02 & 2 & 1,9 \\
\hline 88511 & HD 165173 & O93 & 7.958 & 0.459 & 0.267 & 0.345 & $<0.02$ & 7.958 & 0.459 & 0.267 & 0.345 & 0.03 & $\ldots$ & 1 & 9 \\
\hline 88622 & HD 165401 & SN88 & 6.804 & 0.392 & 0.163 & 0.293 & $<0.02$ & 6.804 & 0.392 & 0.163 & 0.293 & -0.38 & $\ldots$ & 1 & 9 \\
\hline 89215 & $\mathrm{BD}+53640$ & SN88 & 10.348 & 0.474 & 0.261 & 0.141 & 0.020 & 10.282 & 0.458 & 0.266 & 0.137 & -1.18 & 0.08 & 2 & 1,8 \\
\hline 89844 & HD 168443 & O93 & 6.924 & 0.455 & 0.233 & 0.377 & $<0.02$ & 6.924 & 0.455 & 0.233 & 0.377 & 0.08 & 0.01 & 2 & 1,10 \\
\hline 89855 & HD 168060 & O93 & 7.337 & 0.463 & 0.267 & 0.396 & $<0.02$ & 7.337 & 0.463 & 0.267 & 0.396 & 0.28 & $\ldots$ & 1 & 1 \\
\hline 90004 & HD 168746 & O93 & 7.948 & 0.442 & 0.210 & 0.348 & $<0.02$ & 7.948 & 0.442 & 0.210 & 0.348 & -0.08 & 0.01 & 3 & $1,6,10$ \\
\hline 90355 & HD 169822 & O93 & 7.832 & 0.437 & 0.224 & 0.294 & $<0.02$ & 7.832 & 0.437 & 0.224 & 0.294 & -0.12 & $\ldots$ & 1 & 1 \\
\hline 90593 & HD 170469 & $\mathrm{O} 93$ & 8.210 & 0.425 & 0.243 & 0.375 & $<0.02$ & 8.210 & 0.425 & 0.243 & 0.375 & 0.30 & $\ldots$ & 1 & 1 \\
\hline 90656 & HD 170493 & O93 & 8.011 & 0.616 & 0.679 & 0.180 & $<0.02$ & 8.011 & 0.616 & 0.679 & 0.180 & 0.28 & 0.14 & 2 & 1,13 \\
\hline 90864 & HD 171067 & O93 & 7.200 & 0.424 & 0.234 & 0.309 & $<0.02$ & 7.200 & 0.424 & 0.234 & 0.309 & -0.03 & $\ldots$ & 1 & 1 \\
\hline 91360 & HD 171999 & O93 & 8.331 & 0.495 & 0.370 & 0.315 & 0.028 & 8.238 & 0.473 & 0.378 & 0.310 & 0.39 & $\ldots$ & 1 & 3 \\
\hline 91381 & HD 172310 & O93 & 8.413 & 0.441 & 0.227 & 0.245 & $<0.02$ & 8.413 & 0.441 & 0.227 & 0.245 & -0.42 & $\ldots$ & 1 & 1 \\
\hline 91438 & HD 172051 & O93 & 5.852 & 0.418 & 0.216 & 0.277 & $<0.02$ & 5.852 & 0.418 & 0.216 & 0.277 & -0.24 & 0.05 & 3 & $1,12,13$ \\
\hline 91700 & HD 172513 & $\mathrm{O} 93$ & 7.941 & 0.446 & 0.252 & 0.319 & $<0.02$ & 7.941 & 0.446 & 0.252 & 0.319 & -0.01 & $\ldots$ & 1 & 1 \\
\hline 92283 & HD 174080 & $\mathrm{O} 93$ & 7.923 & 0.607 & 0.632 & 0.199 & $<0.02$ & 7.923 & 0.607 & 0.632 & 0.199 & 0.22 & $\ldots$ & 1 & 1 \\
\hline 92918 & HD 175518 & O93 & 7.455 & 0.468 & 0.292 & 0.369 & $<0.02$ & 7.455 & 0.468 & 0.292 & 0.369 & 0.28 & 0.01 & 2 & 1,3 \\
\hline 93007 & HD 176841 & O93 & 7.636 & 0.408 & 0.228 & 0.381 & $<0.02$ & 7.636 & 0.408 & 0.228 & 0.381 & 0.30 & $\ldots$ & 1 & 9 \\
\hline 93341 & HD 230409 & $\mathrm{O} 93$ & 10.104 & 0.440 & 0.201 & 0.208 & 0.034 & 9.993 & 0.414 & 0.210 & 0.202 & -0.86 & $\ldots$ & 1 & 1 \\
\hline 93518 & HD 176982 & O93 & 8.364 & 0.463 & 0.224 & 0.348 & 0.053 & 8.189 & 0.422 & 0.238 & 0.339 & -0.10 & $\ldots$ & 1 & 1 \\
\hline 93858 & HD 177565 & O93 & 6.148 & 0.437 & 0.248 & 0.336 & 0.021 & 6.080 & 0.421 & 0.254 & 0.332 & 0.10 & 0.03 & 3 & $1,2,13$ \\
\hline 93871 & HD 178126 & SN88 & 9.194 & 0.621 & 0.587 & 0.202 & $<0.02$ & 9.194 & 0.621 & 0.587 & 0.202 & -0.88 & $\ldots$ & 1 & 14 \\
\hline \multirow[t]{2}{*}{94075} & HD 178911 & O93 & 8.038 & 0.461 & 0.288 & 0.341 & $<0.02$ & 8.038 & 0.461 & 0.288 & 0.341 & 0.28 & $\ldots$ & 1 & 1 \\
\hline & HD 180890 & O93 & 8.349 & 0.451 & 0.281 & 0.331 & $<0.02$ & 8.349 & 0.451 & 0.281 & 0.331 & 0.17 & $\ldots$ & 1 & 3 \\
\hline 94981 & HD 181655 & O93 & 6.291 & 0.420 & 0.234 & 0.322 & $<0.02$ & 6.291 & 0.420 & 0.234 & 0.322 & 0.06 & $\ldots$ & 1 & 1 \\
\hline 95319 & HD 182488 & O93 & 6.362 & 0.480 & 0.340 & 0.331 & $<0.02$ & 6.362 & 0.480 & 0.340 & 0.331 & 0.19 & 0.05 & 2 & 1,9 \\
\hline 95821 & HD 183650 & O93 & 6.946 & 0.457 & 0.251 & 0.398 & $<0.02$ & 6.946 & 0.457 & 0.251 & 0.398 & 0.31 & $\ldots$ & 1 & 1 \\
\hline 96085 & HD 183870 & O93 & 7.517 & 0.529 & 0.448 & 0.266 & $<0.02$ & 7.517 & 0.529 & 0.448 & 0.266 & 0.05 & $\ldots$ & 1 & 1 \\
\hline 96124 & HD 183877 & SN88 & 7.151 & 0.424 & 0.222 & 0.310 & $<0.02$ & 7.151 & 0.424 & 0.222 & 0.310 & -0.20 & ... & 1 & 1 \\
\hline 96183 & HD 184385 & $\mathrm{O} 93$ & 6.899 & 0.454 & 0.275 & 0.319 & $<0.02$ & 6.899 & 0.454 & 0.275 & 0.319 & 0.15 & $\ldots$ & 1 & 1 \\
\hline 96185 & HD 184499 & SN88 & 6.637 & 0.389 & 0.144 & 0.315 & $<0.02$ & 6.637 & 0.389 & 0.144 & 0.315 & -0.66 & $\ldots$ & 1 & 9 \\
\hline 97219 & HD 187055 & O93 & 9.002 & 0.488 & 0.389 & 0.281 & $<0.02$ & 9.002 & 0.488 & 0.389 & 0.281 & 0.15 & $\ldots$ & 1 & 3 \\
\hline 97420 & HD 187237 & O93 & 6.877 & 0.409 & 0.205 & 0.323 & $<0.02$ & 6.877 & 0.409 & 0.205 & 0.323 & 0.07 & $\ldots$ & 1 & 1 \\
\hline 97769 & HD 188015 & O93 & 8.235 & 0.439 & 0.266 & 0.360 & $<0.02$ & 8.235 & 0.439 & 0.266 & 0.360 & 0.28 & 0.01 & 2 & 1,13 \\
\hline 98020 & HD 188510 & O93 & 8.830 & 0.417 & 0.113 & 0.128 & $<0.02$ & 8.830 & 0.417 & 0.113 & 0.128 & -1.64 & $\ldots$ & 1 & 1 \\
\hline 98066 & HD 188376 & $\mathrm{O} 93$ & 4.700 & 0.458 & 0.258 & 0.359 & $<0.02$ & 4.700 & 0.458 & 0.258 & 0.359 & 0.10 & $\ldots$ & 1 & 7 \\
\hline 98192 & HD 189087 & O93 & 7.886 & 0.483 & 0.319 & 0.298 & $<0.02$ & 7.886 & 0.483 & 0.319 & 0.298 & -0.04 & $\ldots$ & 1 & 9 \\
\hline 98505 & HD 189733 & O93 & 7.665 & 0.527 & 0.442 & 0.272 & $<0.02$ & 7.665 & 0.527 & 0.442 & 0.272 & -0.02 & $\ldots$ & 1 & 15 \\
\hline 98677 & HD 190067 & O93 & 7.146 & 0.452 & 0.233 & 0.287 & $<0.02$ & 7.146 & 0.452 & 0.233 & 0.287 & -0.37 & $\ldots$ & 1 & 1 \\
\hline 98714 & HD 190228 & O93 & 7.311 & 0.482 & 0.264 & 0.306 & 0.028 & 7.219 & 0.460 & 0.272 & 0.301 & -0.18 & 0.13 & 4 & $1,7,6,10$ \\
\hline 98767 & HD 190360 & O93 & 5.742 & 0.461 & 0.275 & 0.372 & $<0.02$ & 5.742 & 0.461 & 0.275 & 0.372 & 0.22 & 0.05 & 3 & $1,9,10$ \\
\hline 98921 & HD 190771 & O93 & 6.172 & 0.413 & 0.222 & 0.336 & $<0.02$ & 6.172 & 0.413 & 0.222 & 0.336 & 0.17 & $\ldots$ & 1 & 1 \\
\hline \multirow[t]{2}{*}{98959} & HD 189567 & SN88 & 6.080 & 0.407 & 0.190 & 0.302 & $<0.02$ & 6.080 & 0.407 & 0.190 & 0.302 & -0.26 & 0.05 & 3 & $1,6,10$ \\
\hline & HD 192020 & O93 & 7.961 & 0.500 & 0.393 & 0.280 & 0.054 & 7.783 & 0.458 & 0.408 & 0.270 & 0.01 & $\ldots$ & 1 & 1 \\
\hline 99711 & HD 192263 & O84 & 7.769 & 0.541 & 0.493 & 0.275 & $<0.02$ & 7.769 & 0.541 & 0.493 & 0.275 & 0.04 & 0.01 & 2 & 1,10 \\
\hline
\end{tabular}


Table B.1. continued.

\begin{tabular}{|c|c|c|c|c|c|c|c|c|c|c|c|c|c|c|c|}
\hline HIP & Name & $\begin{array}{l}\text { Ref. } \\
\text { uvby }\end{array}$ & V & $(b-y)$ & $m_{1}$ & $c_{1}$ & $E(B-V)$ & $V_{0}$ & $(b-y)_{0}$ & $m_{1,0}$ & $c_{1,0}$ & $\langle[\mathrm{Fe} / \mathrm{H}]\rangle$ & $\begin{array}{c}{[\mathrm{Fe} / \mathrm{H}]} \\
\text { range }\end{array}$ & $\mathrm{N}$ & $\begin{array}{c}\text { Ref. } \\
{[\mathrm{Fe} / \mathrm{H}]}\end{array}$ \\
\hline 99729 & HD 192344 & O93 & 7.721 & 0.441 & 0.235 & 0.404 & 0.020 & 7.655 & 0.425 & 0.240 & 0.400 & 0.30 & $\ldots$ & 1 & 1 \\
\hline 100500 & HD 194035 & O93 & 7.134 & 0.450 & 0.229 & 0.389 & $<0.02$ & 7.134 & 0.450 & 0.229 & 0.389 & 0.27 & $\ldots$ & 1 & 1 \\
\hline 100568 & HD 193901 & SN88 & 8.660 & 0.383 & 0.099 & 0.221 & $<0.02$ & 8.660 & 0.383 & 0.099 & 0.221 & -1.23 & $\ldots$ & 1 & 1 \\
\hline 100792 & HD 194598 & SN88 & 8.354 & 0.344 & 0.091 & 0.269 & $<0.02$ & 8.354 & 0.344 & 0.091 & 0.269 & -1.33 & $\ldots$ & 1 & 9 \\
\hline 101597 & HD 196201 & O93 & 8.495 & 0.456 & 0.256 & 0.296 & $<0.02$ & 8.495 & 0.456 & 0.256 & 0.296 & -0.14 & $\ldots$ & 1 & 1 \\
\hline 101997 & HD 196761 & O93 & 6.376 & 0.440 & 0.251 & 0.269 & $<0.02$ & 6.376 & 0.440 & 0.251 & 0.269 & -0.27 & 0.10 & 4 & $1,12,6,10$ \\
\hline 102018 & HD 196800 & SN88 & 7.210 & 0.388 & 0.196 & 0.380 & $<0.02$ & 7.210 & 0.388 & 0.196 & 0.380 & 0.16 & $\ldots$ & 1 & 1 \\
\hline 103077 & HD 198802 & O93 & 6.395 & 0.416 & 0.196 & 0.381 & $<0.02$ & 6.395 & 0.416 & 0.196 & 0.381 & 0.04 & $\ldots$ & 1 & 1 \\
\hline 103096 & HD 199305 & O93 & 8.554 & 0.911 & 0.518 & 0.201 & $<0.02$ & 8.554 & 0.911 & 0.518 & 0.201 & -0.28 & $\ldots$ & 1 & 14 \\
\hline 103458 & HD 199288 & SN88 & 6.523 & 0.386 & 0.143 & 0.269 & $<0.02$ & 6.523 & 0.386 & 0.143 & 0.269 & -0.63 & $\ldots$ & 1 & 1 \\
\hline 103654 & HD 199190 & SN88 & 6.872 & 0.397 & 0.202 & 0.383 & $<0.02$ & 6.872 & 0.397 & 0.202 & 0.383 & 0.15 & $\ldots$ & 2 & 1,15 \\
\hline 104092 & HD 200779 & O93 & 8.267 & 0.690 & 0.747 & 0.115 & $<0.02$ & 8.267 & 0.690 & 0.747 & 0.115 & 0.02 & $\ldots$ & 1 & 12 \\
\hline 104318 & HD 201219 & O93 & 8.013 & 0.444 & 0.256 & 0.331 & $<0.02$ & 8.013 & 0.444 & 0.256 & 0.331 & 0.15 & $\ldots$ & 1 & 1 \\
\hline 104436 & HD 199509 & O94 & 6.988 & 0.396 & 0.179 & 0.265 & $<0.02$ & 6.988 & 0.396 & 0.179 & 0.265 & -0.31 & $\ldots$ & 1 & 1 \\
\hline 104659 & HD 201891 & SN88 & 7.386 & 0.363 & 0.094 & 0.261 & $<0.02$ & 7.386 & 0.363 & 0.094 & 0.261 & -1.10 & 0.05 & 3 & $1,4,9$ \\
\hline 104809 & HD 201989 & O93 & 7.385 & 0.430 & 0.237 & 0.320 & $<0.02$ & 7.385 & 0.430 & 0.237 & 0.320 & 0.12 & $\ldots$ & 1 & 1 \\
\hline 104903 & HD 202206 & O93 & 7.920 & 0.437 & 0.249 & 0.388 & $<0.02$ & 7.920 & 0.437 & 0.249 & 0.388 & 0.34 & 0.02 & 3 & $1,6,10$ \\
\hline 105038 & HD 202575 & O93 & 7.896 & 0.579 & 0.568 & 0.206 & $<0.02$ & 7.896 & 0.579 & 0.568 & 0.206 & 0.04 & $\ldots$ & 1 & 1 \\
\hline 105152 & HD 202751 & O93 & 8.159 & 0.563 & 0.540 & 0.262 & $<0.02$ & 8.159 & 0.563 & 0.540 & 0.262 & -0.10 & $\ldots$ & 1 & 1 \\
\hline 105388 & HD 202917 & O93 & 8.597 & 0.435 & 0.234 & 0.299 & $<0.02$ & 8.597 & 0.435 & 0.234 & 0.299 & 0.11 & $\ldots$ & 1 & 1 \\
\hline 106696 & HD 205390 & O94 & 7.144 & 0.514 & 0.401 & 0.263 & $<0.02$ & 7.144 & 0.514 & 0.401 & 0.263 & -0.17 & $\ldots$ & 1 & 1 \\
\hline 107022 & HD 205536 & O93 & 7.057 & 0.462 & 0.279 & 0.318 & $<0.02$ & 7.057 & 0.462 & 0.279 & 0.318 & -0.03 & $\ldots$ & 1 & 1 \\
\hline 107070 & HD 206374 & O93 & 7.445 & 0.430 & 0.245 & 0.289 & $<0.02$ & 7.445 & 0.430 & 0.245 & 0.289 & -0.05 & $\ldots$ & 1 & 1 \\
\hline 108158 & HD 207700 & SN88 & 7.437 & 0.434 & 0.225 & 0.368 & $<0.02$ & 7.437 & 0.434 & 0.225 & 0.368 & 0.04 & $\ldots$ & 1 & 1 \\
\hline 108736 & HD 208998 & SN88 & 7.133 & 0.371 & 0.149 & 0.338 & $<0.02$ & 7.133 & 0.371 & 0.149 & 0.338 & -0.32 & $\ldots$ & 1 & 1 \\
\hline 108774 & HD 209393 & O93 & 7.970 & 0.417 & 0.234 & 0.262 & $<0.02$ & 7.970 & 0.417 & 0.234 & 0.262 & -0.17 & $\ldots$ & 1 & 1 \\
\hline 108870 & HD 209100 & O93 & 4.682 & 0.588 & 0.605 & 0.202 & $<0.02$ & 4.682 & 0.588 & 0.605 & 0.202 & 0.04 & $\ldots$ & 2 & 6,10 \\
\hline 109144 & HD 209875 & SN88 & 7.244 & 0.353 & 0.152 & 0.393 & $<0.02$ & 7.244 & 0.353 & 0.152 & 0.393 & -0.11 & $\ldots$ & 1 & 1 \\
\hline 109169 & HD 211681 & O93 & 8.105 & 0.449 & 0.257 & 0.395 & 0.048 & 7.947 & 0.412 & 0.270 & 0.387 & 0.45 & $\ldots$ & 1 & 1 \\
\hline 109355 & HD 210312 & O93 & 8.639 & 0.434 & 0.245 & 0.382 & $<0.02$ & 8.639 & 0.434 & 0.245 & 0.382 & 0.28 & $\ldots$ & 1 & 1 \\
\hline 109461 & $\mathrm{BD}+224567$ & O93 & 9.191 & 0.542 & 0.454 & 0.276 & $<0.02$ & 9.191 & 0.542 & 0.454 & 0.276 & -0.18 & $\ldots$ & 1 & 8 \\
\hline 109821 & HD 210918 & O93 & 6.220 & 0.415 & 0.195 & 0.323 & $<0.02$ & 6.220 & 0.415 & 0.195 & 0.323 & -0.11 & $\ldots$ & 2 & 1,2 \\
\hline 109836 & HD 211080 & O93 & 7.838 & 0.443 & 0.243 & 0.424 & 0.027 & 7.749 & 0.422 & 0.250 & 0.419 & 0.39 & $\ldots$ & 1 & 1 \\
\hline 109926 & HD 211472 & O93 & 7.500 & 0.476 & 0.342 & 0.300 & $<0.02$ & 7.500 & 0.476 & 0.342 & 0.300 & 0.06 & $\ldots$ & 1 & 9 \\
\hline 110508 & HD 212291 & O93 & 7.909 & 0.435 & 0.226 & 0.284 & $<0.02$ & 7.909 & 0.435 & 0.226 & 0.284 & -0.12 & $\ldots$ & 1 & 1 \\
\hline 110649 & HD 212330 & O93 & 5.319 & 0.424 & 0.206 & 0.364 & $<0.02$ & 5.319 & 0.424 & 0.206 & 0.364 & 0.05 & $\ldots$ & 1 & 1 \\
\hline 110843 & HD 212708 & O93 & 7.494 & 0.451 & 0.262 & 0.373 & $<0.02$ & 7.494 & 0.451 & 0.262 & 0.373 & 0.28 & 0.02 & 2 & 1,15 \\
\hline 110996 & HD 213042 & O93 & 7.621 & 0.619 & 0.666 & 0.183 & $<0.02$ & 7.621 & 0.619 & 0.666 & 0.183 & 0.20 & $\ldots$ & 1 & 5 \\
\hline 111148 & HD 213519 & O93 & 7.702 & 0.416 & 0.175 & 0.338 & $<0.02$ & 7.702 & 0.416 & 0.175 & 0.338 & 0.00 & $\ldots$ & 1 & 1 \\
\hline 111978 & HD 214759 & O94 & 7.415 & 0.476 & 0.328 & 0.331 & $<0.02$ & 7.415 & 0.476 & 0.328 & 0.331 & 0.22 & 0.10 & 3 & $1,2,15$ \\
\hline 112229 & HD 215257 & SN88 & 7.410 & 0.357 & 0.116 & 0.310 & $<0.02$ & 7.410 & 0.357 & 0.116 & 0.310 & -0.66 & $\ldots$ & 1 & 4 \\
\hline 113137 & HD 216437 & O93 & 6.056 & 0.422 & 0.215 & 0.394 & $<0.02$ & 6.056 & 0.422 & 0.215 & 0.394 & 0.22 & $\ldots$ & 1 & 1 \\
\hline 113283 & HD 216803 & O93 & 6.450 & 0.622 & 0.630 & 0.177 & $<0.02$ & 6.450 & 0.622 & 0.630 & 0.177 & 0.09 & 0.01 & 2 & 6,10 \\
\hline 113421 & HD 217107 & O93 & 6.175 & 0.455 & 0.295 & 0.374 & $<0.02$ & 6.175 & 0.455 & 0.295 & 0.374 & 0.38 & 0.03 & 4 & $1,7,6,10$ \\
\hline 113948 & HD 217958 & O93 & 8.041 & 0.416 & 0.215 & 0.408 & $<0.02$ & 8.041 & 0.416 & 0.215 & 0.408 & 0.31 & ... & 1 & 1 \\
\hline 114622 & HD 219134 & O93 & 5.545 & 0.571 & 0.552 & 0.268 & $<0.02$ & 5.545 & 0.571 & 0.552 & 0.268 & 0.11 & 0.23 & 4 & $1,7,12,9$ \\
\hline 114699 & HD 219077 & O94 & 6.131 & 0.478 & 0.255 & 0.350 & $<0.02$ & 6.131 & 0.478 & 0.255 & 0.350 & -0.12 & $\ldots$ & 1 & 1 \\
\hline 115087 & HD 219709 & O94 & 7.502 & 0.399 & 0.197 & 0.326 & $<0.02$ & 7.502 & 0.399 & 0.197 & 0.326 & -0.01 & $\ldots$ & 1 & 2 \\
\hline 115445 & HD 220339 & O93 & 7.793 & 0.510 & 0.416 & 0.253 & $<0.02$ & 7.793 & 0.510 & 0.416 & 0.253 & -0.31 & $\ldots$ & 1 & 1 \\
\hline 116085 & HD 221354 & SN88 & 6.750 & 0.500 & 0.362 & 0.342 & $<0.02$ & 6.750 & 0.500 & 0.362 & 0.342 & 0.01 & $\ldots$ & 1 & 1 \\
\hline 116250 & HD 221420 & O94 & 5.818 & 0.427 & 0.225 & 0.441 & $<0.02$ & 5.818 & 0.427 & 0.225 & 0.441 & 0.34 & 0.01 & 2 & 1,15 \\
\hline 116421 & HD 221830 & SN88 & 6.862 & 0.397 & 0.159 & 0.327 & $<0.02$ & 6.862 & 0.397 & 0.159 & 0.327 & -0.40 & ... & 1 & 1 \\
\hline 116745 & HD 222237 & O94 & 7.068 & 0.561 & 0.523 & 0.239 & $<0.02$ & 7.068 & 0.561 & 0.523 & 0.239 & -0.24 & 0.08 & 3 & $1,6,10$ \\
\hline 116763 & HD 222335 & O94 & 7.185 & 0.477 & 0.327 & 0.282 & $<0.02$ & 7.185 & 0.477 & 0.327 & 0.282 & -0.14 & 0.05 & 3 & $1,6,10$ \\
\hline 116852 & HD 222480 & O93 & 7.117 & 0.423 & 0.197 & 0.407 & $<0.02$ & 7.117 & 0.423 & 0.197 & 0.407 & 0.18 & 0.01 & 2 & 1,15 \\
\hline 116984 & HD 222697 & O93 & 8.675 & 0.473 & 0.290 & 0.371 & $<0.02$ & 8.675 & 0.473 & 0.290 & 0.371 & 0.16 & $\ldots$ & 1 & 1 \\
\hline 117159 & $\mathrm{BD}+284634$ & SN88 & 8.382 & 0.504 & 0.381 & 0.290 & $<0.02$ & 8.382 & 0.504 & 0.381 & 0.290 & -0.04 & $\ldots$ & 1 & 8 \\
\hline 117320 & HD 223171 & SN88 & 6.888 & 0.414 & 0.203 & 0.382 & $<0.02$ & 6.888 & 0.414 & 0.203 & 0.382 & 0.12 & $\ldots$ & 2 & 1,15 \\
\hline 117427 & HD 223315 & O93 & 8.763 & 0.452 & 0.263 & 0.397 & $<0.02$ & 8.763 & 0.452 & 0.263 & 0.397 & 0.30 & $\ldots$ & 1 & 1 \\
\hline 117526 & HD 223498 & O93 & 8.336 & 0.456 & 0.272 & 0.366 & $<0.02$ & 8.336 & 0.456 & 0.272 & 0.366 & 0.23 & $\ldots$ & 1 & 1 \\
\hline 117668 & HD 223691 & O93 & 7.867 & 0.445 & 0.228 & 0.325 & $<0.02$ & 7.867 & 0.445 & 0.228 & 0.325 & -0.17 & $\ldots$ & 1 & 1 \\
\hline 117953 & HD 224156 & O93 & 7.750 & 0.458 & 0.267 & 0.326 & $<0.02$ & 7.750 & 0.458 & 0.267 & 0.326 & -0.03 & $\ldots$ & 1 & 1 \\
\hline \multirow[t]{2}{*}{118115} & HD 224383 & SN88 & 7.896 & 0.403 & 0.200 & 0.341 & $<0.02$ & 7.896 & 0.403 & 0.200 & 0.341 & -0.04 & $\ldots$ & 1 & 1 \\
\hline & PLX 1219 & SN88 & 11.515 & 0.522 & 0.293 & 0.144 & $<0.02$ & 11.515 & 0.522 & 0.293 & 0.144 & -1.67 & $\ldots$ & 1 & 8 \\
\hline
\end{tabular}

